\title{
Geomorphic Characteristics and Classification of Duluth-Area Streams, Minnesota
}
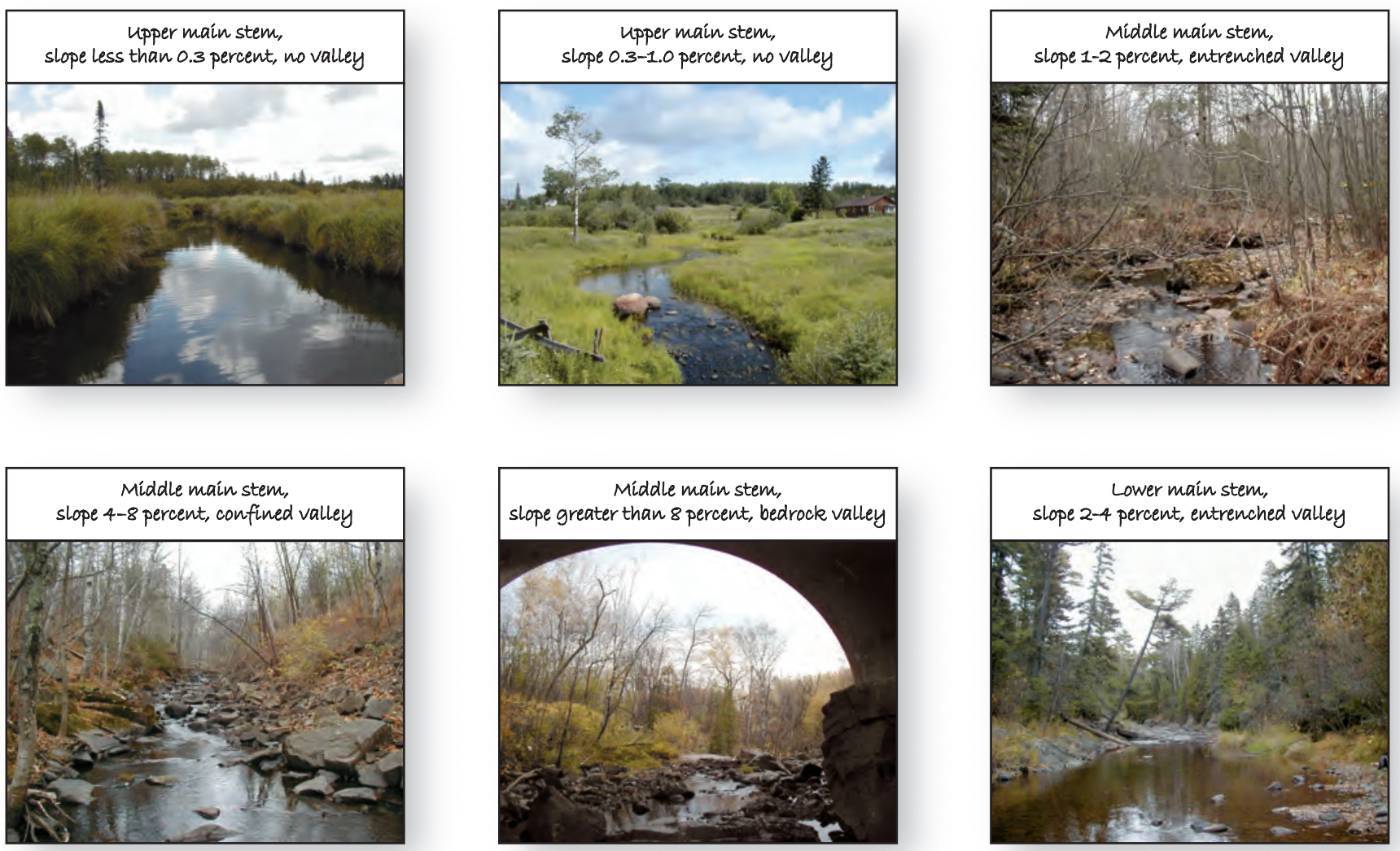

Scientific Investigations Report 2006-5029 


\section{Geomorphic Characteristics and Classification of Duluth-Area Streams, Minnesota}

By Faith A. Fitzpatrick, Marie C. Peppler, Michele M. DePhilip, and Kathy E. Lee

In cooperation with the City of Duluth, Minnesota

Scientific Investigations Report 2006-5029 


\section{U.S. Department of the Interior \\ Gale A. Norton, Secretary}

\section{U.S. Geological Survey \\ P. Patrick Leahy, Acting Director}

\section{U.S. Geological Survey, Reston, Virginia: 2006}

For product and ordering information:

World Wide Web: http://www.usgs.gov/pubprod

Telephone: 1-888-ASK-USGS

For more information on the USGS--the Federal source for science about the Earth, its natural and living resources, natural hazards, and the environment:

World Wide Web: http://www.usgs.gov

Telephone: 1-888-ASK-USGS

Any use of trade, product, or firm names is for descriptive purposes only and does not imply endorsement by the U.S. Government.

Although this report is in the public domain, permission must be secured from the individual copyright owners to reproduce any copyrighted materials contained within this report. 


\section{Contents}

Conversion Factors, Vertical Datum, and Abbreviated Units of Measurement ....................... vi

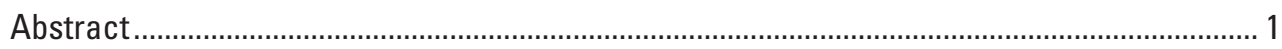

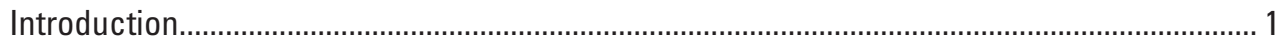

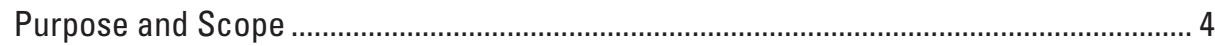

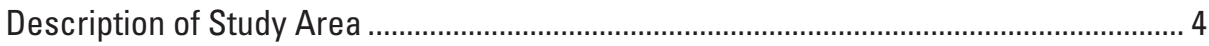

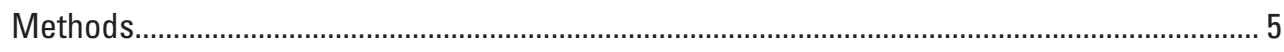

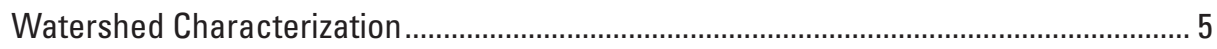

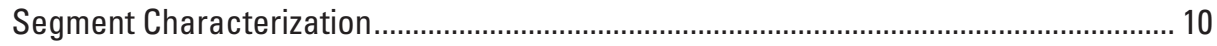

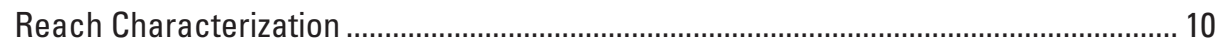

Rapid Field Assessments..................................................................................... 10

Intensive Field Surveys .................................................................................. 12

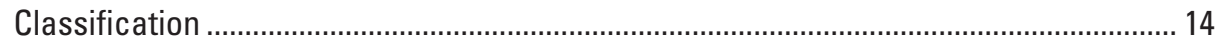

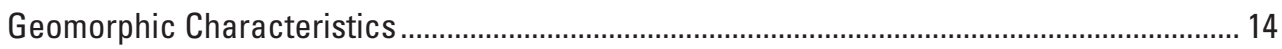

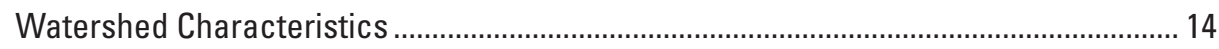

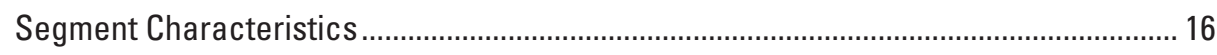

Reach Characteristics and Processes....................................................................... 32

Potential Response and Sensitivity to Disturbance ....................................................... 45

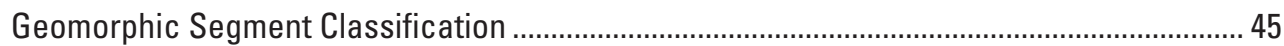

Headwater Channels with Gentle Slopes ....................................................................... 49

Upper Main Stems with Moderate Slopes .................................................................. 49

Middle Main Stems with Gentle or Moderate Slopes ...................................................... 49

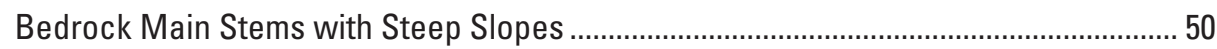

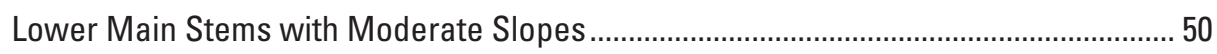

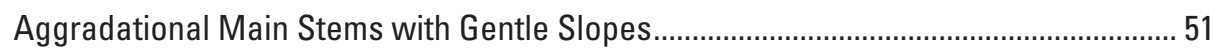

Tributaries with Moderate to Steep Slopes................................................................ 51

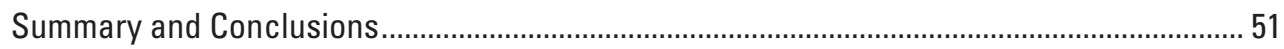

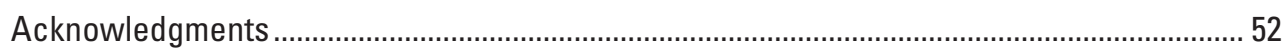

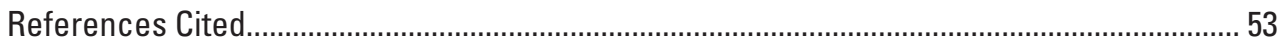




\section{Figures}

1. Map showing study area and sampling sites in the Duluth, Minn., area, 2003-2004

2. Plan view and longitudinal profile of geomorphic zones within a stream network

3. Diagram showing spatial hierarchy of watershed, stream-segment, and stream-reach scales.

4-7. Maps showing:

4. Bedrock geology of the Duluth, Minn., area ................................................ 6

5. Surficial geology and geomorphology of the Duluth, Minn., area ...................... 7

6. Land cover in the Duluth, Minn., area............................................................. 8

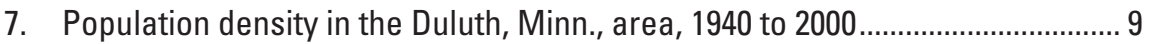

8. Diagram showing valley development and valley types for Duluth-area streams, Minn

9. Graphs showing longitudinal profiles of streams in the $\boldsymbol{A}$, southwestern, and $\boldsymbol{B}$, northeastern parts of the Duluth, Minn., area ............................................ 15

10. Diagram showing hierarchical classification of channel types in the Duluth, Minn., area

11. Graphs showing longitudinal profiles for $\boldsymbol{A}$, Lester River and $\boldsymbol{B}$, Miller Creek, in the Duluth, Minn., area.

12. Photographs of rapid assessment sites on tributaries affected by bedrock: $\boldsymbol{A}$, site 46 on Coffee Creek, $\boldsymbol{B}$, site 2 on Stewart Creek tributary, $\boldsymbol{C}$, site 47 on Merritt Creek tributary, and $\boldsymbol{D}$, site 5 on Keene Creek tributary, Duluth, Minn., area

13. Maps showing geomorphic segment classification for Duluth-area streams, Minn., ( $\boldsymbol{A}$, northern portion and $\boldsymbol{B}$, southern portion) 


\section{Tables}

1. Land-cover characteristics for Duluth-area watersheds, Minn. ............................. 13

2. Conditions at the mouths of Duluth-area streams, Minn........................................... 16

3. Number of stream segments in each slope/drainage-network position category for Duluth-area streams, Minn.................................................................. 17

4. Number of stream segments in each slope/valley type category for Duluth-area streams, Minn.

5. Geomorphic characteristics of stream segments for Duluth-area streams,

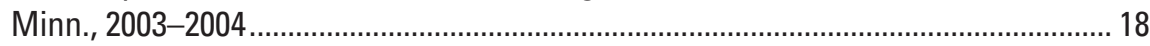

6. Explanation of abbreviations used in tables for segment and reach characteristics in this study of Duluth-area streams, Minn.

7. Reach characteristics at rapid assessment and intensive survey field sites for Duluth-area streams, Minn., 2003-2004

8. Potential channel types in each slope/valley category for Duluth-area streams, Minn. 44

9. Geomorphic segment classification scheme for streams in the Duluth, Minn., area based on slope, drainage-network position, geologic setting, and valley type 


\section{Conversion Factors, Vertical Datum, and Abbreviated Units of Measurement}

\begin{tabular}{lcl}
\hline \multicolumn{1}{c}{ Multiply } & By & \multicolumn{1}{c}{ To obtain } \\
\hline inch (in.) & Length & \\
foot (ft) & 2.54 & centimeter $(\mathrm{cm})$ \\
& 0.3048 & meter $(\mathrm{m})$ \\
\hline square mile $\left(\mathrm{mi}^{2}\right)$ & Area & \\
\hline & 2.590 & square kilometer $\left(\mathrm{km}^{2}\right)$ \\
\hline cubic foot per second $\left(\mathrm{ft}^{3} / \mathrm{s}\right)$ & Flow rate & \\
\hline
\end{tabular}

Temperature in degrees Fahrenheit $\left({ }^{\circ} \mathrm{F}\right)$ may be converted to degrees Celsius $\left({ }^{\circ} \mathrm{C}\right)$ as follows:

$$
{ }^{\circ} \mathrm{C}=\left({ }^{\circ} \mathrm{F}-32\right) / 1.8
$$

Vertical coordinate information is referenced to the North American Vertical Datum of 1927 (NAVD 27).

Horizontal coordinate information is referenced to the North American Datum of 1983 (NAD 83).

Altitude, as used in this report, refers to distance above the vertical datum. 


\title{
Geomorphic Characteristics and Classification of Duluth-Area Streams, Minnesota
}

\author{
By Faith A. Fitzpatrick, Marie C. Peppler, Michele M. DePhilip', and Kathy E. Lee
}

\section{Abstract}

In 2003 and 2004, a geomorphic assessment of streams in 20 watersheds in the Duluth, Minn., area was conducted to identify and summarize geomorphic characteristics, processes, disturbance mechanisms, and potential responses to disturbance. Methods used to assess the streams included watershed characterization, descriptions of segment slopes and valley types, historical aerial photograph interpretation, and rapid field assessments and intensive field surveys of stream reaches. Geomorphic conditions were summarized into a segment-scale classification with 15 categories mainly based on drainage-network position and slope, and, secondarily, based on geologic setting, valley type, and dominant geomorphic processes. Main causes of geomorphic disturbance included historical logging and agriculture, and ongoing urban development, human-caused channel alterations, road and storm sewer drainage, ditching, hiking trails, and gravel pits or quarries. Geomorphic responses to these disturbances are dependent on a combination of drainage-network position, slope, and geologic setting. Geologic setting is related to drainage-network position because the geologic deposits parallel the Lake Superior shoreline. Headwater streams in large watersheds flow over glacial deposits above altitudes of about 1,200 feet (ft). Headwater tributaries and upper main stems have ditch-like channels with gentle slopes and no valleys. Urban development and road drainage cause increased runoff and flood peaks in these segments resulting in channel widening. Below about 1,200 ft, mainstem segments generally are affected by bedrock type and structure and have steep slopes and confined or entrenched valleys. Increases in flood peaks do not cause incision or widening in the bedrock-controlled valleys; instead, the flow and scour areas are expanded. Feeder tributaries to these main stems have steep, confined valleys and may be sources for sediment from urban areas, road runoff, or storm sewer outfalls. Main-stem segments near the glacial deposits/surficial bedrock contact (1,000-1,200 ft) have the most potential for response to disturbance because they tend to have narrow valleys with sandy glacial lakeshore deposits and moderate slopes. Increases in flood peaks (from upstream increases in runoff) increase the potential for landslides and mass wasting from valley sides as well as channel widening.

\section{Introduction}

Streams in the Duluth, Minn., area (fig. 1) have been affected by a variety of historical land uses but remain highly valued for their natural resources. Streams and riparian areas contain important habitat for endangered or threatened aquatic and terrestrial species. Understanding the geomorphic conditions and processes of streams within a stream network or watershed framework is important because they relate to the overall water and aquatic resource quality of the stream and to the plant and animal communities and habitat adjacent to the stream. In 20032004, the U.S. Geological Survey (USGS), in cooperation with the city of Duluth, studied the geomorphic characteristics and processes that affect the physical conditions of Duluth-area streams.

The Duluth Natural Areas Program (DNAP) is based on a city ordinance designed to protect and preserve the natural heritage of Duluth and the surrounding area. The program strives to protect plant and animal communities, habitat for special species, natural water features, important bird habitat areas, and landscapes. Data on stream geomorphic conditions provide a basis for evaluating the rarity and quality of natural resources nominated to DNAP for their natural water features. An assessment of geomorphic conditions within the stream channels is important

${ }^{1}$ The Nature Conservancy, Great Lakes Program, Chicago, Ill. 


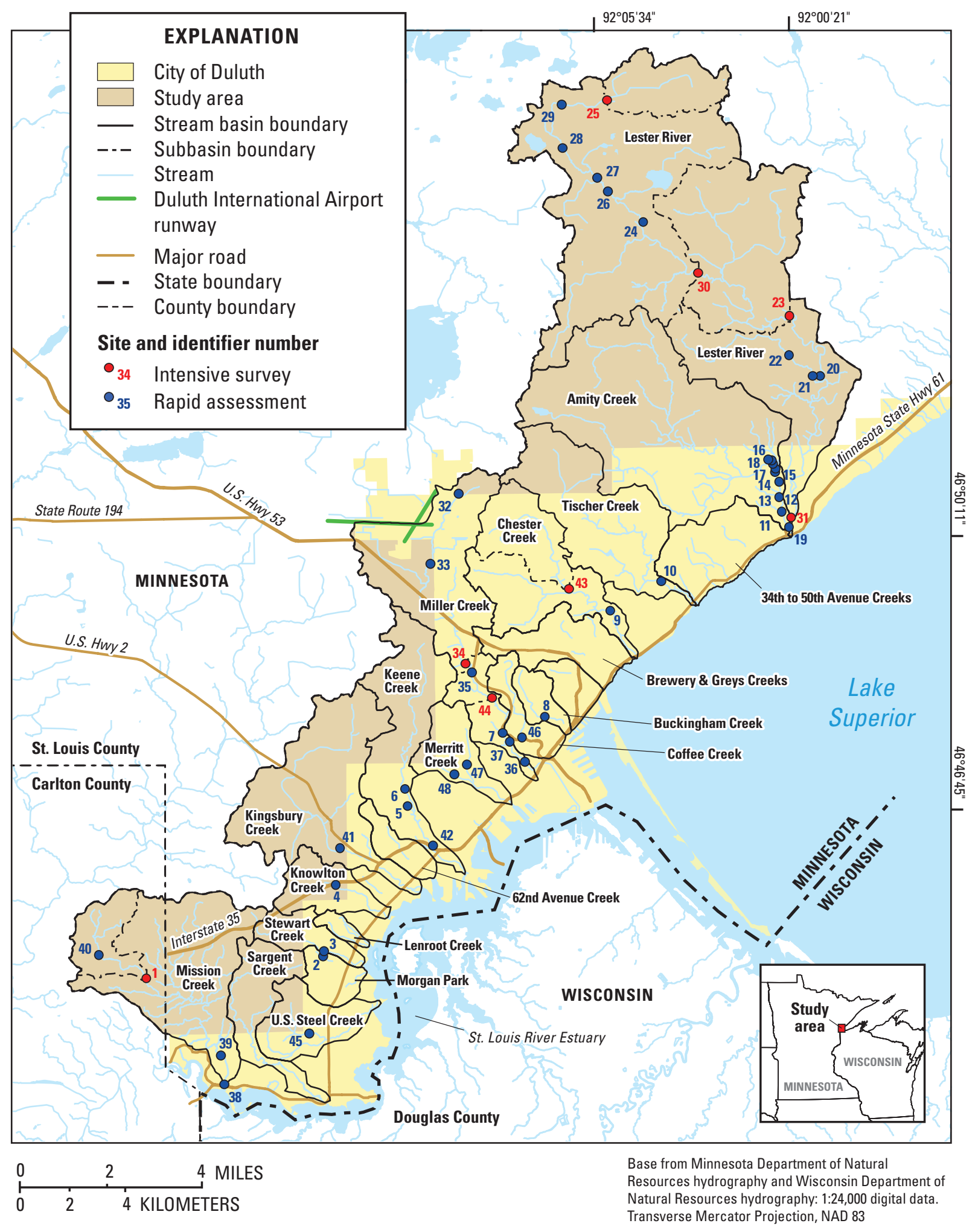

Figure 1. Study area and sampling sites in the Duluth, Minn., area, 2003-2004. Airport runway location from Minnesota Department of Transportation (2001). 
for understanding the extent to which natural factors and land-use changes have affected geomorphic attributes, such as bed and channel configurations, and the likelihood that geomorphic conditions may change in the future.

A thorough stream geomorphic assessment includes a description of how channel, flood-plain, and valley landforms are spatially and temporally affected by regional and local geology, topography, climate, vegetative cover, and human modifications. Stream networks developed in homogeneous geologic deposits commonly consist of an erosion zone (where sediment and runoff are generated), a transition zone (were sediment and water are transferred through with no net gain or loss of material), and a deposition zone (where sediment from upstream erosion is dropped out of transport) (Schumm, 1977). A typical longitudinal profile of a stream illustrates how the slope of a stream typically changes from relatively steep in the erosion zone near its headwaters to gentle in the depositional zone near its mouth (Hack, 1960) (fig. 2). An understanding of sediment sources, transport, and deposition and their links to stream network position is key to understanding geomorphic responses to present and future disturbances.

Three common spatial scales used to identify geomorphic characteristics and processes are a watershed or landscape scale, a segment scale, and a reach or channel scale (fig. 3). The segment scale is a section of stream bounded
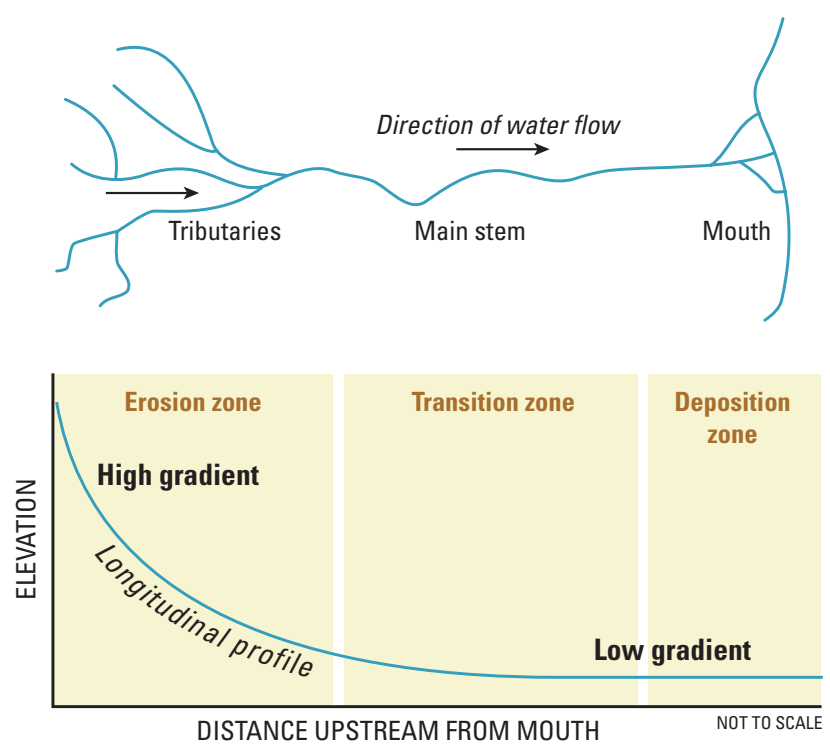

Figure 2. Plan view and longitudinal profile of geomorphic zones within a stream network (modified from Schumm, 1977)

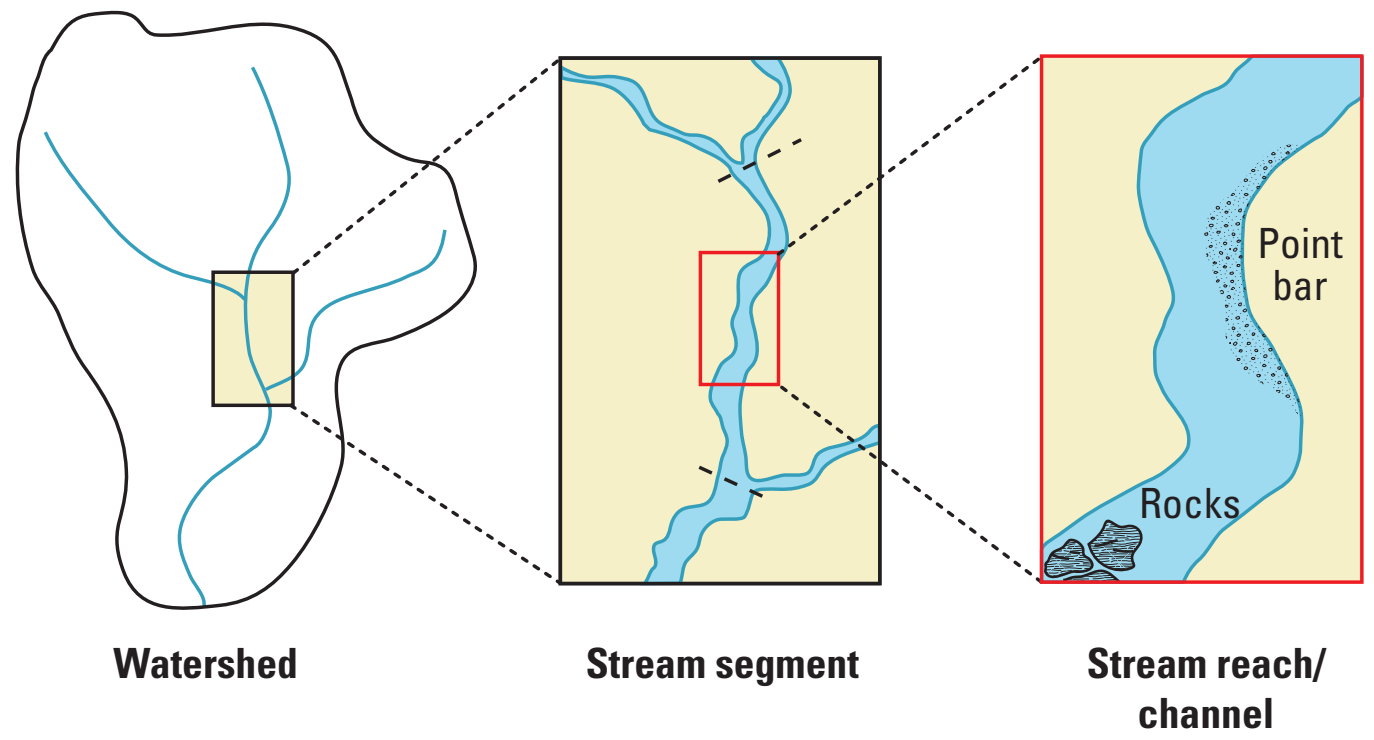

Figure 3. Spatial hierarchy of watershed, stream-segment, and stream-reach scales (modified from Fitzpatrick and others, 1998). 
by confluences or physical or chemical discontinuities, such as major landforms, changes in slope, or point-source discharges. The reach scale is a length of stream chosen to illustrate a set of uniform channel features and represents the typical sampling unit used for field-based measurements of geomorphic characteristics. Reach-scale geomorphic conditions and processes can be used to infer conditions and processes for a given segment.

A hierarchical channel classification system, based on geomorphic processes, was developed for mountain streams in the Pacific Northwest to help interpret channel morphology, assess geomorphic conditions, and predict responses to natural and human-caused disturbance (Montgomery and Buffington, 1993; 1997). In the Montgomery and Buffington (1997) classification, valleys were classified into three major types based on the predominant geologic materials in the valley-colluvial, bedrock, and alluvial (confined, moderately confined, and unconfined, respectively). Colluvial deposits mainly originate from landslides and mass wasting, whereas alluvial deposits are from transport and depositional processes related to modern rivers. Channels were classified into seven types-colluvial, bedrock, cascade, step-pool, plane-bed, pool-riffle, and dune-ripple. The channel types can be related to drainage-network position, underlying geology, and slope. The Montgomery and Buffington $(1993 ; 1997)$ classification is not commonly used in the Midwest because streams have relatively gentle slopes compared to mountain streams. However, tributaries along the north shore of Lake Superior drain bedrock bluff areas and have slopes that exceed 8 percent along some reaches. Bohle (2002) developed a modified version of this classification to assess geomorphic conditions for streams along the north shore of Lake Superior in Minnesota. Bohle (2002) classified stream segments into eight geomorphic map units (GMUs)—boulder/bedrock, main-stem boulder rapid, gravel-dominated pool-riffle, forced pool-riffle plane-bed, large woody debris/boulder step, tributary boulder plane-bed or rapid, beaver dam step-pool, and lacustrine/riverine wetland.

The major objectives of the present geomorphic study of Duluth-area streams are to describe geomorphic characteristics, identify major geomorphic processes and factors contributing to geomorphic characteristics, identify historical changes in channel morphology and planform (plan view of the channel pattern), and develop a segment-scale classification based on similar geomorphic characteristics, processes, and responses to disturbance. The assessment and classification will provide baseline conditions and can be used as a tool for evaluating the quality of Duluth's natural water features. Scientists, city managers, and citizens involved in the Duluth Natural Areas Program can use results from this study to help protect, preserve, and restore resources and help guide management activities. In addition to its social and conservation benefits, this study will advance the understanding of present and historical geomorphic conditions of Duluth-area streams, of other north- and south-shore tributaries to Lake Superior, and of other streams in similar hydrologic settings.

\section{Purpose and Scope}

The purpose of this report is to provide an assessment and classification of the geomorphic characteristics, processes, and responses to disturbances of streams in the vicinity of Duluth, Minnesota. The scope of this report includes: 1) description of the current geomorphic characteristics, 2) identification of major geomorphic processes, 3) identification of factors contributing to current geomorphic characteristics and expected future trends in characteristics, 4) interpretations of historical changes in channel morphology and planform, and 5) development of a segment-scale classification based on geomorphic processes. Geomorphic characterizations and the classification system were based on watershed-, segment-, and reach-scale measurements and observations collected in 2003 and 2004.

Any attempt to classify the myriad geomorphic characteristics and processes that can occur in urbanizing or urban streams with disturbed watersheds that have been altered by land clearing, channelization, and ditching into discrete categories will have limitations and problems. Therefore, the main goal of the classification portion of the study, for use with Duluth-area streams only, is to help show the importance of linking geomorphic characteristics and processes of a specific reach of stream in the context of its position within the landscape and geologic setting.

\section{Description of Study Area}

Streams in the Duluth area generally flow perpendicular to the shoreline of Lake Superior, from northwest to southeast (fig. 1). Altitudes of watersheds range from $1,400 \mathrm{ft}$ (above NAVD 27) in the headwaters to $602 \mathrm{ft}$ at Lake Superior. Most of the altitude change occurs along the middle and lower main stems as streams flow over bedrock bluffs and outcrops near the shore of Lake Superior. 
The climate of the Duluth area is characterized by cold snowy winters, cool or moderately warm summers, and evenly distributed precipitation (Eichenlaub, 1979). Large variations from average weather can occur because of the proximity of Duluth to Lake Superior. Average annual precipitation is 29-30 in., with $18 \mathrm{in}$. of average annual evapotranspiration and 10-12 in. of runoff (Young and Skinner, 1974). Mean daily maximum air temperatures range from $22^{\circ} \mathrm{F}$ in January to $78^{\circ} \mathrm{F}$ in July (Eichenlaub, 1979).

Bedrock is near or at the land surface in the Duluth area and mainly consists of Proterozoic volcanic and igneous rocks, intrusions, and sedimentary rocks (Miller and others, 2002) (fig. 4). Nomenclature for geologic names of bedrock units follows that of the Minnesota Geological Survey. Bedrock type and faulting affects the pattern and density of the stream networks. Right-angle bends in the channel and stream confluences are indicative of underlying contacts between bedrock units or faulting. The Duluth Complex and miscellaneous other intrusive rocks form a prominent steep rocky bluff that parallels the shoreline of the St. Louis River Estuary and Lake Superior.

Quaternary surficial deposits in the Duluth area are generally in the upper parts of most of the watersheds but thin or absent from the middle main stems where bedrock is exposed at the land surface (fig. 5). Glacial deposits in the watersheds are from the Late Wisconsinan Lake Superior Lobe (Goebel and others, 1983). Glacial deposits in the headwaters and the upper parts of the watersheds above about 1,200 ft consist of supraglacial drift composed of sandy loamy till of the Cromwell Formation (Goebel and others, 1983; University of Minnesota-Duluth Geology Department and others, 1997; Hobbs, 2004). Later glacial readvances resulted in the deposition of silty loam till of the Lakewood Member, clay loam to silty clay loam till of the Moose Lake Member, and clay till of the informally named Knife River member, all of the Barnum Formation (Hobbs, 2004). In the Duluth area, the Lakewood Member is at altitudes from about 1,150 to $1,200 \mathrm{ft}$. The Moose Lake Member is at altitudes from about 1,100 to $1,150 \mathrm{ft}$. The Knife River member is at altitudes up to about 1,050 $\mathrm{ft}$. Shoreline features from wave action and beaches are present from about 1,020 to $1,100 \mathrm{ft}$ and were caused by multiple phases of a glacial lake in the southwest part of Lake Superior during glacial retreats in the Late Wisconsinan and early Holocene (Leverett, 1929; Hobbs, 2004).

Land cover in the Duluth area consists of a mix of mainly urban land (developed/barren), forest, and shrub
(Minnesota Department of Natural Resources, Division of Forestry, 2002) (fig. 6). Urban land is concentrated in Duluth and along transportation corridors leading into Duluth (fig. 1). Categories of marsh, lowland shrub, and lowland forest are in wetland settings.

Population density in 2000 for the area surrounding the studied streams ranged from greater than 5,000 people/ $\mathrm{mi}^{2}$ near the center of Duluth to less than 250 people/ $\mathrm{mi}^{2}$ in rural areas (U.S. Census Bureau, 2000) (fig. 7). Comparison of population density maps for 1940, 1960, 1980, and 2000 shows how urban development has spread through the watershed of Miller Creek and along the shore of Lake Superior north of Duluth over the last 20 years (U.S. Census Bureau, 2000; Van Riper, unpublished data).

\section{Methods}

The methods used to assess the geomorphic characteristics of Duluth-area streams consisted of a variety of techniques that included data collection and interpretation at watershed, segment, and reach scales.

\section{Watershed Characterization}

Drainage boundaries were delineated for the 20 studied watersheds and also for subwatersheds of nine intensive survey sites to examine differences among watershed characteristics. Digital drainage boundaries from the USGS (Minnesota Water Science Center, written commun., 2003) were used and (or) updated based on visual interpretations from USGS 7.5-minute topographic maps. Drainage boundaries were overlaid with thematic maps of bedrock geology (Miller and others, 2002) (fig. 4), Quaternary deposits (University of Minnesota-Duluth Geology Department and others, 1997) (fig. 5), and land cover (Minnesota Department of Natural Resources, Division of Forestry, 2002) (fig. 6) by use of a geographic information system (GIS). These overlays were used to summarize differences among the watersheds for geologic setting and land cover (mainly the amount of urbanization).

Longitudinal profiles were constructed for 15 mainstem streams. Stream lengths were measured with a map measurer between contour lines on USGS 7.5-minute topographic maps. Longitudinal profiles are used to identify changes in slope that are related to geologic features or spatial position within the stream network. 


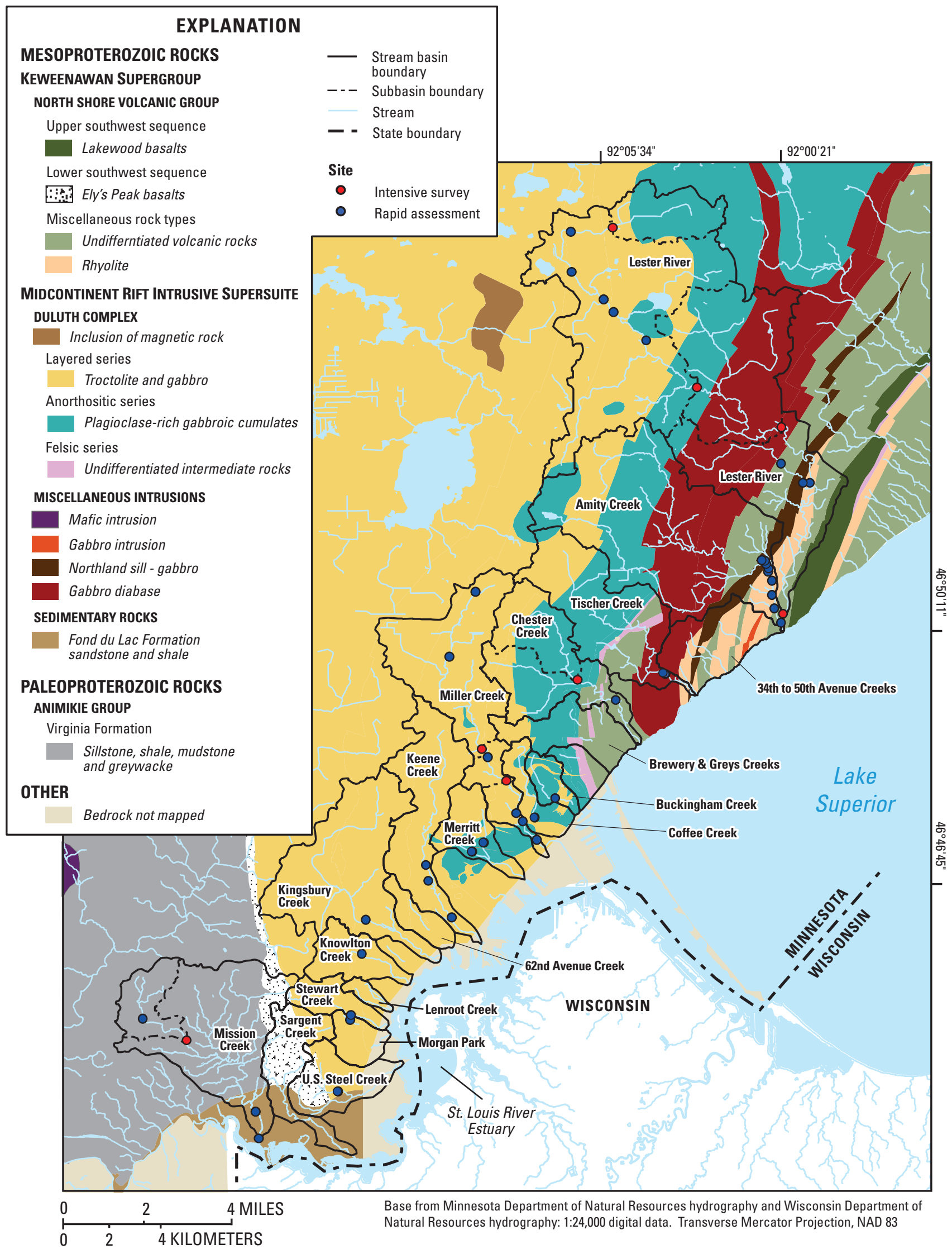

Figure 4. Bedrock geology of the Duluth, Minn., area (from Miller and others, 2002). 


\section{EXPLANATION}

_ Stream basin boundary

-.- Subbasin boundary Stream

- - State boundary

Site

- Intensive survey

- Rapid assessment

\section{Holocene}

Alluvium, silt and sand with some gravel; hummocky

Organic deposits, peat; level

\section{Late Wisconsin}

Lacustrine deposits associated with Lake Duluth; clayey or sandy

VI//A Outwash, sand or gravel; rolling or steep

Ice contact, sand or gravel; hummocky or steep

Till plain, lake-modified, clay; rolling or undulating

Till plain, clayey; rolling Supraglacial drift complex, loamy/sandy; steep

\section{Proterozoic}

Scoured bedrock uplands; rolling Bedrock

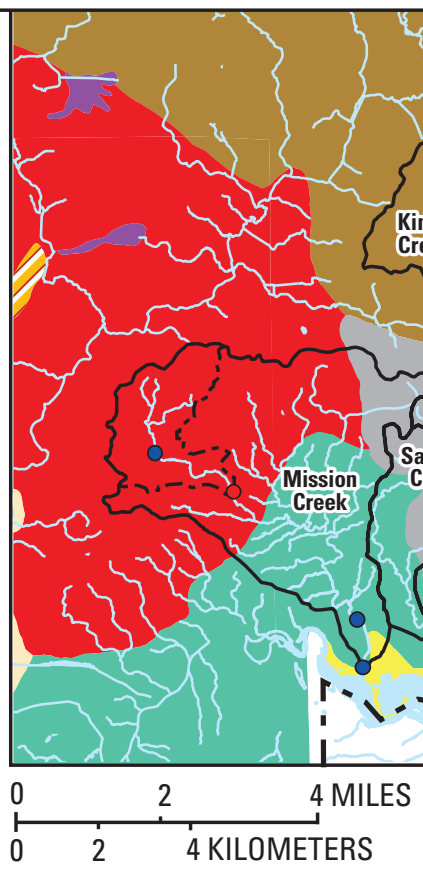

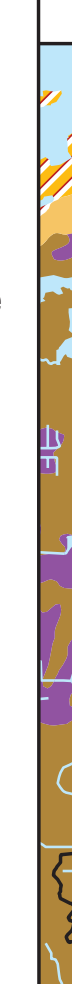

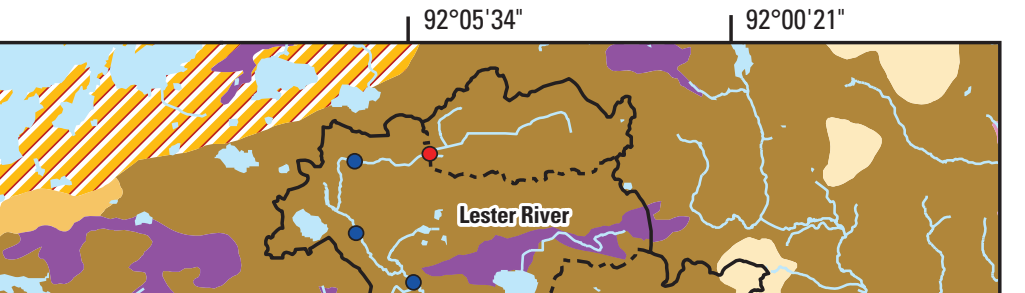




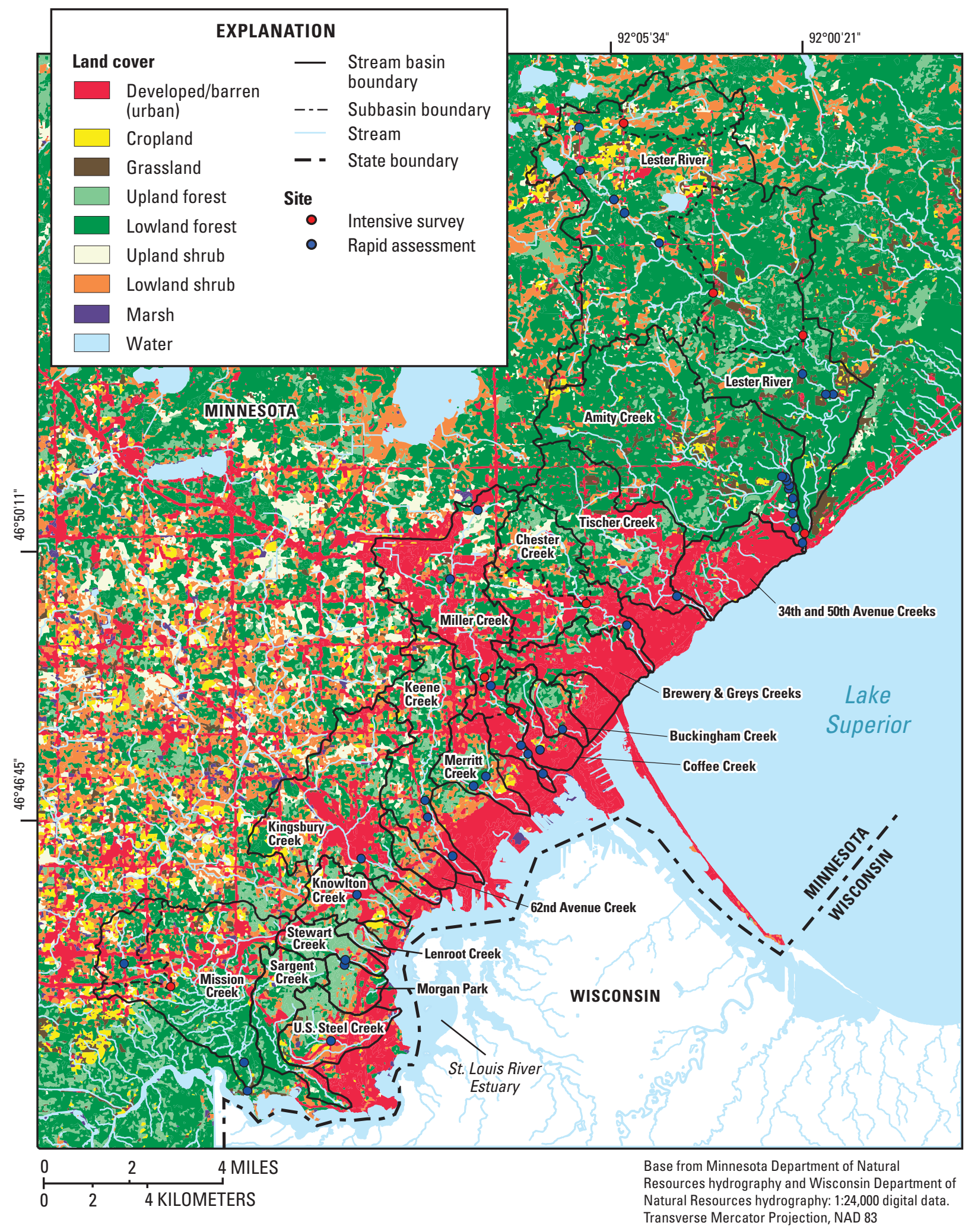

Figure 6. Land cover in the Duluth, Minn., area (from Minnesota Department of Natural Resources, Division of Forestry, 2002). 

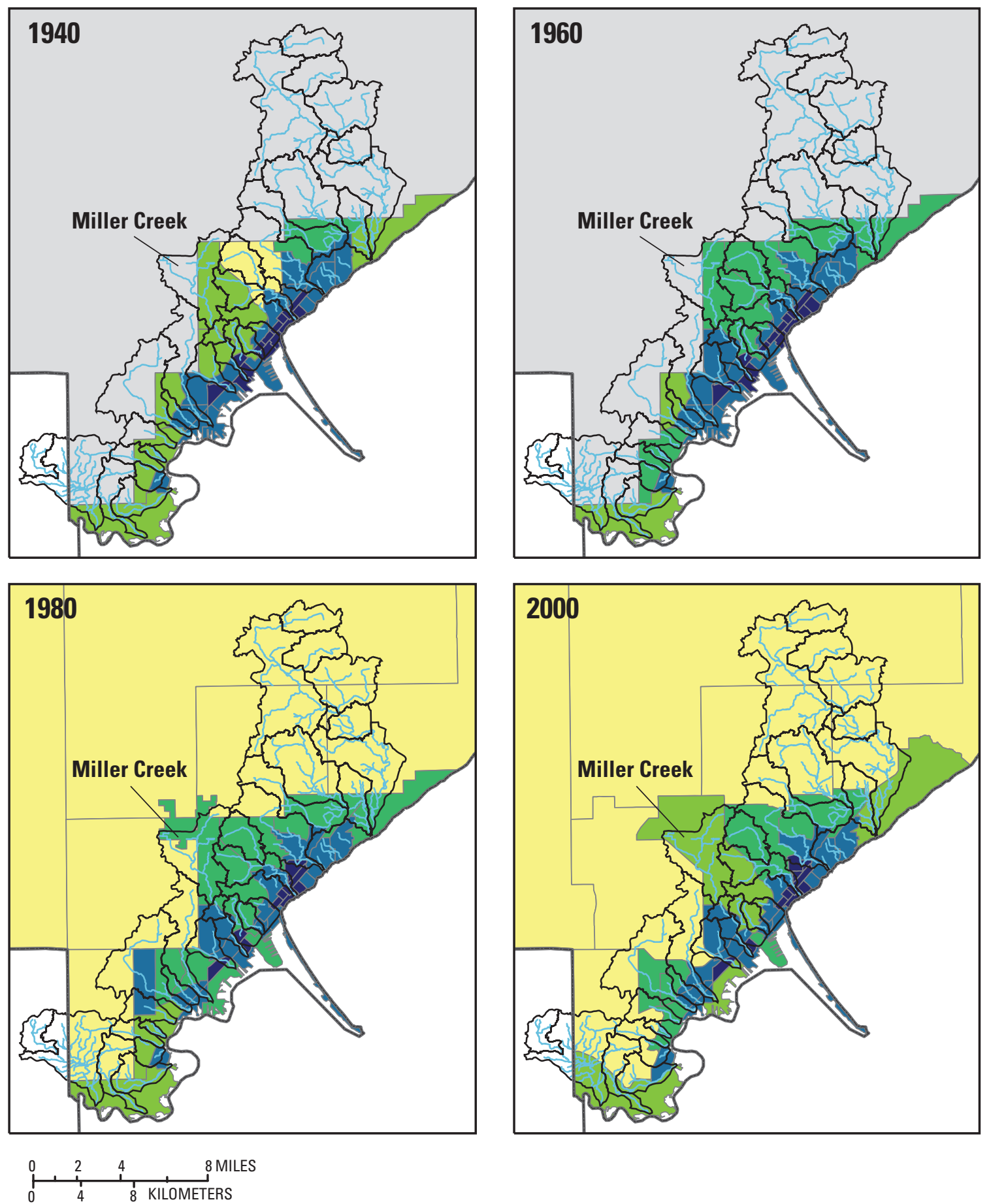

\section{EXPLANATION}

Stream basin
boundary
Stream
- County boundary
Census tract
boundary

Population density in people per square mile

\begin{tabular}{|c|}
\hline $1-250$ \\
\hline $251-500$ \\
\hline $501-1,000$ \\
\hline $1,001-5,000$ \\
\hline Greater than 5,000 \\
\hline
\end{tabular}

Figure 7. Population density in the Duluth, Minn., area, 1940 to 2000 (U.S. Census Bureau, 2000; Van Riper, unpublished data). 


\section{Segment Characterization}

Streams were first divided into segments with similar slope and valley confinement, similar to methods described in Montgomery and Buffington (1998). Slope and valley confinement characterizations initially were based on measurements and observations from USGS 7.5-minute topographic maps. Each segment was assigned a unique identification code. Segments were categorized by slope: less than 0.3 percent, 0.3 to 1 percent, greater than 1 to 2 percent, greater than 2 to 4 percent, greater than 4 to 8 percent, or greater than 8 percent. Each segment was classified as confined (valley width less than 2 times the channel width), moderately confined (valley width between 2 and 4 times the channel width), or unconfined (valley width greater than 4 times the channel width). Valley confinement was difficult to estimate from topographic maps; these initial characterizations were confirmed during the reach field surveys and changed, if necessary. Channelsegment boundaries also were confirmed in the field and changed if necessary.

After the field assessments were done, it was determined that valleys in the Duluth area fit into four groups based on modified categories assigned to valleys for Lake Superior tributaries in the western part of the Upper Peninsula of Michigan (Hack, 1965) (fig. 8). Headwater streams in the upstream parts of the watersheds have no valleys, are unconfined $(\mathrm{N})$, and flow over glacial deposits. As drainage area increases and altitude decreases, the stream valleys are confined (C) V-shaped valleys as they cut through the steep, post-glacial shoreline areas, faults, or bedrock. Continuing downstream, the valleys become well developed but stream meanders follow the valley meanders (E). This type of valley and stream meander pattern was called "entrenched" by Hack (1965). The stream-meander pattern for this valley type tends to be irregular and determined by the intersection of the stream and valley with bedrock outcrops, post-glacial lake shorelines, or glacial meltwater valleys. In entrenched valleys, channels commonly intersect valley sides, which combined with enough flow and shear stress, can cause bluff and terrace erosion. Near the mouths, valleys tend to be wide and unconfined and stream-meander patterns are alluvial (A). The valleys are wider than the meander belt and alluvial stream meanders typically are a reflection of fluvial processes of runoff and sediment transport conditions.

Aerial photographs from 1939-40, 1956, 1997, and 2002 were used to compare historical and current channel and riparian conditions for the stream segments. Channel width, canopy pattern, type and size of riparian vegetation on each stream bank, stability and position of gravel bars, location of eroding banks, wood loading, and channel position were noted. Riparian vegetation composition was described as deciduous (greater than 70 percent deciduous), coniferous (greater than 70 percent coniferous) or mixed. Riparian vegetation age was described as young (less than 10 years old), mature (between 10 and 40 years old), or old (greater than 40 years old). Riparian vegetation density was described as sparse (greater than 50 percent open ground) or dense (greater than 50 percent forested).

Drainage-network position was assigned to each segment based on the longitudinal profiles and 7.5-minute topographic maps. Main stems were divided into upper, middle, and lower categories, corresponding to main-stem length. Tributary categories were assigned based on the category assignment of the main stem at their confluence.

Predominant categories of bedrock type, glacial landforms/deposits, and land cover were assigned to each segment based on the thematic overlays (Miller and others, 2002; University of Minnesota-Duluth Geology Department and others, 1997; Minnesota Department of Natural Resources, Division of Forestry, 2002; respectively). Bedrock types included sedimentary, gabbro rocks, felsic and mafic volcanic rocks, intrusions, and bedrock buried by glacial deposits. In addition to thematic overlay of bedrock geology from Miller and others (2002), bedrock types along segments were visually checked by use of the nondigital 1:24,000-scale maps of bedrock types and outcrops in Schwartz (1949). Glacial landform/deposits categories were supraglacial drift, outwash, ice-contact, glacial lake, post-glacial shoreline/beach, bedrock surface, till plain, and undifferentiated. Dominant land cover included urban, developing urban, agriculture, forest, wetland, grass, shrubland, and roads.

\section{Reach Characterization}

\section{Rapid Field Assessments}

Rapid assessments of geomorphic characteristics and processes (mainly observation-based) were done at 48 sites from July 2003 through May 2004 (fig. 1). These sites were on segments with a range in slope and valley type. Rapid assessments were based on qualitative and quantitative field methods described in Thorne (1998) for identifying geomorphic characteristics and processes 

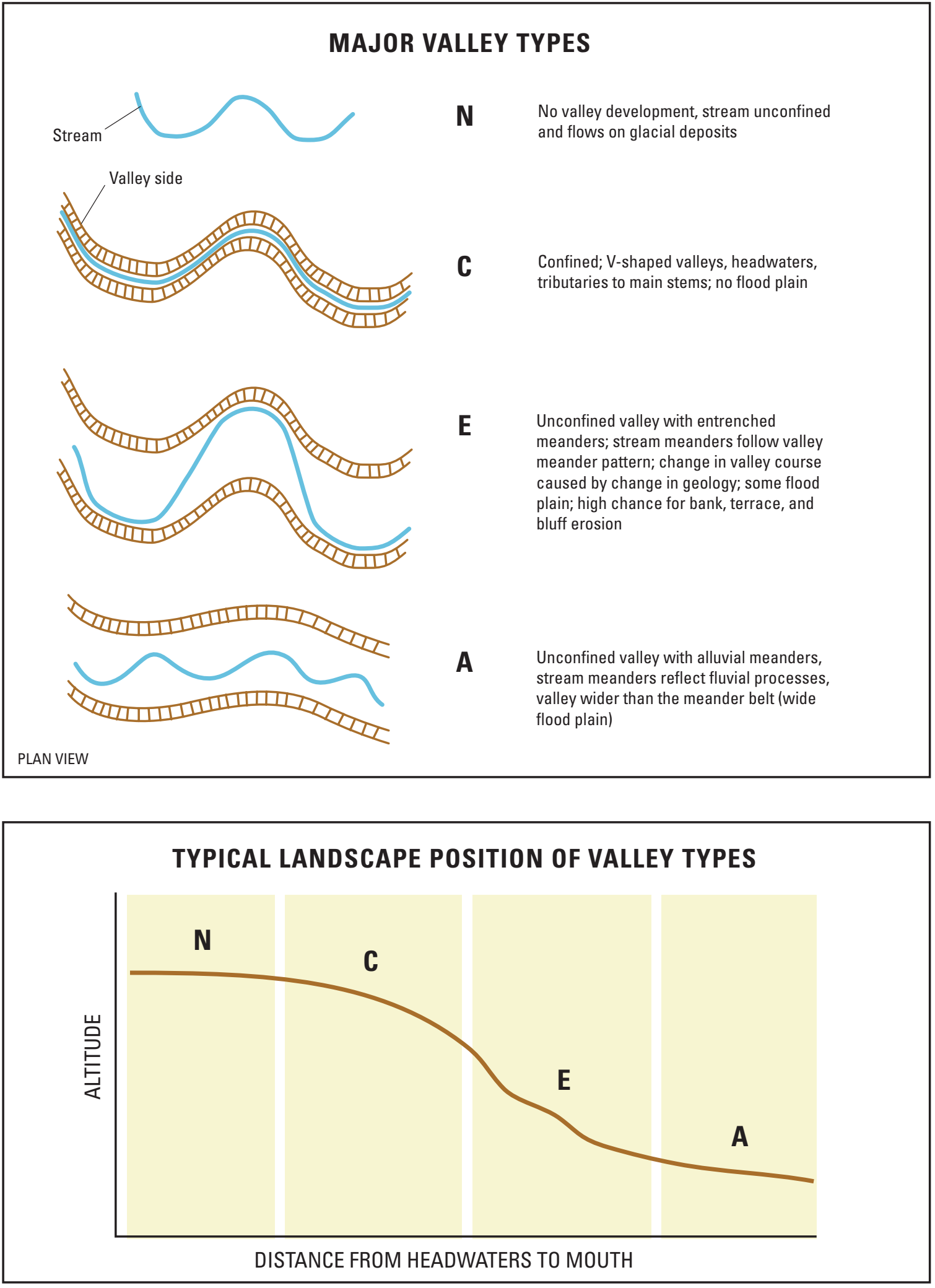

Figure 8. Valley development and valley types for Duluth-area streams, Minn. 
of streams for multi-disciplinary reconnaissance studies. Thorne's (1998) field methods include observations and measurements of riparian vegetation and geology, valley and flood-plain characteristics, and channel characteristics in relation to valley conditions. Channel morphology, bar formation, substrate, and controls on incision and lateral migration were measured and described. Bank conditions and interpretations also were measured and described. Descriptions also included type of road crossing, potential fish-passage problems through culverts, and any other potential local effects to the stream channel. Culverts are common in Duluth-area streams, and if inappropriately constructed, can be obstructions to fish migration (Gibson and others, 2005). Photographs of the streams were taken during the rapid assessments. Rapid assessment data were used to compare observed characteristics to predictions of dominant geomorphic channel processes based on the geomorphic segment classification.

\section{Intensive Field Surveys}

Intensive field surveys were done at nine sites in July, August, and October 2003. These were a subset of sites where rapid field assessments were done (fig. 1). The most common slope and valley types were selected. In addition, sites were concentrated in three watersheds-Mission Creek, Miller Creek, and Lester River-to describe how geomorphic characteristics and processes changed along the longitudinal profiles and also to identify upstream to downstream linkages in geomorphic processes. Assessments included qualitative and quantitative geomorphic assessment of valley, channel, and bank conditions; channel cross-section surveys, substrate measurements, cores of overbank sediment, and measurements of large woody debris (LWD) and pool area. Channel and bank measurements were based on reach-scale descriptions. Reach lengths were based on spacing of riffles, steps, or bedrock falls and ranged from 85 to $273 \mathrm{ft}$ with an average reach length of $170 \mathrm{ft}$. Channel types were identified as bedrock, colluvial, cascade, step-pool, pool-riffle, plane-bed, or wetland based on definitions from Montgomery and Buffington (1998).

Two channel cross sections were surveyed at each intensive site with an automatic level (Harrelson and others, 1994). A global positioning system was used to verify the location of each cross section. Semi-permanent benchmarks (rebar stakes) were established at each cross section. The cross sections were in riffles. Water-surface and channel slope were measured along the stream distance between the two cross sections.

Quantitative measurements of coarse substrate size in riffles were made by means of Wolman pebble counts (Wolman, 1954). One riffle was chosen (out of the two with cross-section surveys) to conduct the survey. Pebble selection techniques followed Fitzpatrick and others (1998) and the diameter of the b-axis of 100 pebbles were measured and recorded in a field book. If sand or smallersized particles were encountered, these particles were tallied as "sand" or "fines".

Overbank sedimentation was measured through exploratory coring with a hand-held soil probe and all cores were described for texture and color in the field by use of the U.S. Department of Agriculture textural triangle and color chart (Munsell Color, 1975; Soil Survey Staff, 1951). Field grading of texture was done by rubbing soil between the fingers (Milfred and others, 1967). Photographs were taken in each reach.

The number, length, and average diameter of woody debris were measured in each reach. Geomorphic function and source for the wood were recorded. Possible functions included pool scour, bank stability, bar stabilizer, sediment trap, or step former. Possible sources for the wood included side slope, upstream, or bank. All wood within the bankfull channel was measured that was larger than 0.1 $\mathrm{ft}$ in diameter and $1 \mathrm{ft}$ in length. There is no minimum size standard for LWD. The relatively small minimum size of wood counted in this study (as compared to other studies) was selected to maximize options for comparing LWD in Duluth streams to LWD surveys from other sources.

The area and depth of pools were measured in each reach. Pools were identified by having relatively deep, slow-moving water, and fine-grained bed material compared to the rest of the reach. Pool function also was recorded and included free, LWD, or bedrock/boulder/bank forced.

Channel widths recorded in Government Land Office (GLO) Survey notes from the Duluth area (1857, 1858, 1868, and 1870) were compared to channel and wetted widths measured in cross-section surveys in 2003 to quantify potential channel-width changes at intensive sites. In the GLO surveys, channel widths at section line crossings were measured in links, and 1 link is equivalent to 0.66 $\mathrm{ft}$. It is best to compare GLO surveyed widths at multiplesection line crossings to obtain a sense of data accuracy. For this study, it was assumed that changes greater than $+3 \mathrm{ft}$ were appreciable. 
Table 1. Land-cover characteristics for Duluth-area watersheds, Minn. (based on Minnesota Gap Analysis Program Classification Level 3; Minnesota Department of Natural Resources, Division of Forestry, 2002).

$[<$, less than $]$

\begin{tabular}{|c|c|c|c|c|c|c|c|c|c|c|}
\hline Watershed & $\begin{array}{c}\text { Drainage } \\
\text { area } \\
\text { (square } \\
\text { miles) }\end{array}$ & $\begin{array}{c}\text { Developed/ } \\
\text { barren } \\
\text { (urban) } \\
\text { (percent) }\end{array}$ & $\begin{array}{l}\text { Cropland } \\
\text { (percent) }\end{array}$ & $\begin{array}{l}\text { Grassland } \\
\text { (percent) }\end{array}$ & $\begin{array}{c}\text { Upland } \\
\text { forest } \\
\text { (percent) }\end{array}$ & $\begin{array}{c}\text { Lowland } \\
\text { forest } \\
\text { (percent) }\end{array}$ & $\begin{array}{c}\text { Upland } \\
\text { shrub } \\
\text { (percent) }\end{array}$ & $\begin{array}{l}\text { Lowland } \\
\text { shrub } \\
\text { (percent) }\end{array}$ & $\begin{array}{c}\text { Marsh } \\
\text { (percent) }\end{array}$ & $\begin{array}{c}\text { Water } \\
\text { (percent) }\end{array}$ \\
\hline Mission Creek & 10.6 & 21 & 4 & 2 & 10 & 46 & 3 & 12 & 1 & $<1$ \\
\hline Sargent Creek & 3.1 & 11 & 1 & 1 & 31 & 46 & 1 & 9 & $<1$ & 0 \\
\hline U.S. Steel Creek & 2.8 & 34 & 3 & 3 & 22 & 16 & $<1$ & 19 & 2 & 0 \\
\hline Morgan Park Creek & 1.3 & 24 & 1 & 1 & 43 & 19 & 0 & 13 & 0 & 0 \\
\hline Stewart Creek & 1.5 & 16 & 1 & 1 & 47 & 25 & 1 & 9 & $<1$ & 0 \\
\hline Lenroot Creek & .4 & 17 & $<1$ & 1 & 62 & 6 & 0 & 15 & 0 & 0 \\
\hline Knowlton Creek & 2.2 & 31 & 3 & 2 & 24 & 18 & 5 & 17 & $<1$ & 0 \\
\hline Kingsbury Creek & 9.0 & 34 & 2 & 1 & 8 & 27 & 8 & 19 & 1 & $<1$ \\
\hline 62nd Avenue Creek & 1.2 & 45 & 1 & 2 & 13 & 14 & 1 & 24 & 1 & 0 \\
\hline Keene Creek & 6.0 & 30 & 1 & 2 & 11 & 32 & 6 & 18 & $<1$ & 0 \\
\hline Merritt Creek & 2.2 & 38 & 1 & 1 & 19 & 24 & 1 & 16 & $<1$ & 0 \\
\hline Miller Creek & 9.3 & 60 & $<1$ & 1 & 3 & 21 & 6 & 8 & $<1$ & $<1$ \\
\hline Buckingham Creek & 1.1 & 54 & 0 & 1 & 17 & 19 & $<1$ & 8 & $<1$ & 0 \\
\hline Coffee Creek & 1.6 & 66 & 1 & 0 & 8 & 20 & $<1$ & 4 & 0 & 0 \\
\hline $\begin{array}{l}\text { Brewery and Greys } \\
\text { Creeks }\end{array}$ & 3.0 & 75 & $<1$ & 0 & 6 & 16 & $<1$ & 3 & 0 & 0 \\
\hline Chester Creek & 6.7 & 33 & 1 & 1 & 9 & 36 & 7 & 12 & 1 & $<1$ \\
\hline Tischer Creek & 7.3 & 34 & $<1$ & 3 & 11 & 44 & 2 & 5 & $<1$ & $<1$ \\
\hline $\begin{array}{l}\text { 34th to 50th Ave. } \\
\text { Creeks }\end{array}$ & 3.6 & 80 & $<1$ & $<1$ & 5 & 11 & $<1$ & 2 & $<1$ & $<1$ \\
\hline Amity Creek & 16.7 & 7 & 2 & 9 & 7 & 69 & 1 & 5 & $<1$ & $<1$ \\
\hline Lester River & 36.0 & 3 & 2 & 5 & 7 & 68 & 2 & 11 & $<1$ & 1 \\
\hline
\end{tabular}




\section{Classification}

The segment-scale classification developed for Duluth-area streams was based in part on Montgomery and Buffington's (1998) classification. Segments were grouped into 15 categories based on drainage-network position, slope, geologic setting (including a combination of bedrock and glacial landform features), valley type, dominant geomorphic processes, and potential responses to disturbance. The classification was based on results from the rapid field assessments and intensive field surveys. Channel types, geomorphic processes, and interpretations of sensitivity to disturbance summarized from the reach data were extrapolated to other segments with similar drainagenetwork position, slope, geologic setting, and disturbance mechanisms. Interpretations of potential for geomorphic change due to disturbance are based on observations and evidence for historical geomorphic change as well broad assumptions about the effects of slope and parent material on geomorphic processes.

\section{Geomorphic Characteristics}

Geomorphic characteristics of Duluth-area streams and the processes responsible for them are determined by topography and slope, drainage-network position, geologic setting (watershed, segment, and reach scales), valley type, base-level controls, vegetation, and human-caused alterations and disturbance. Current (2005) geomorphic processes reflect short- and long-term responses to disturbances. The source for the disturbance may be upstream or downstream of a particular segment or reach of interest.

\section{Watershed Characteristics}

Watershed characteristics help to determine the amount and timing of runoff and sediment from uplands to channels. The 20 studied watersheds are generally small and range in size from less than $1 \mathrm{mi}^{2}$ (Lenroot Creek) to $36 \mathrm{mi}^{2}$ (Lester River) (fig. 1; table 1). All of the streams are underlain by igneous rocks except Mission Creek (fig. 4). Bedrock crops out at the land surface through much of the watersheds but is buried by glacial deposits in the upper parts of the larger watersheds (fig. 5). There is little infiltration and potential for runoff is high where bedrock crops out or is thinly covered by glacial deposits. The Duluth Gabbro complex underlies many of the streams from Stewart Creek northeast to the upper watershed of Tischer Creek. Lester River and Amity Creek are underlain by felsic and mafic volcanic units interspersed with gabbro and mafic intrusions. These bedrock units have different rates of erosion. The sedimentary rocks that underlie the Mission Creek watershed are less resistant to erosion than the volcanics, gabbro complex, or the intrusions. Thus, the Mission Creek drainage pattern is denser and more dendritic than patterns for the other watersheds.

Land cover in the studied watersheds is predominantly composed of urban land (developed/barren), forest, or shrub (fig. 6, table 1). Percentages of urban land range from 3 percent in the Lester River watershed to 80 percent in the 34th to 50th Ave. Creeks watershed. Urban land is most concentrated near the mouths along the shoreline of the St. Louis River estuary and Lake Superior; however, urban land extends throughout many of the watersheds, especially those that are bisected by major transportation corridors along U.S. State Highway 53, U.S. Interstate 35, and U.S. State Highway 2 (figs. 1 and 6). Urban watersheds have higher potential for increased runoff rates and volumes and for changes in sediment inputs compared to forested watersheds. Watersheds with more than 50 percent urban land include Miller Creek, Coffee Creek, Buckingham Creek, Brewery and Greys Creeks, and 34th to 50th Ave. Creeks. Seven urban streams near downtown Duluth run underground at their mouths (table 2). Watersheds that are predominantly forest include Mission Creek, Sargent Creek, Morgan Park Creek, Stewart Creek, Lenroot Creek, Amity Creek, and Lester River (table 1). Watersheds that have had noticeable urban development from 1980 to 2000 include Mission Creek, Morgan Park Creek, Stewart Creek, Lenroot Creek, Miller Creek, and Lester River (fig. 7). Geomorphic effects from urban development are concerns for Miller Creek, Chester Creek, and Amity Creek (Camp Dresser and McKee, Inc., 2000).

Duluth-area main stems have complex longitudinal profiles (fig. 9) that are very different from the typical longitudinal profile shown in figure 2 for geologically old landscapes with homogeneous geologic deposits. Irregularities in the Duluth stream profiles are attributed to differences in the erodibility of geologic materials, extent of landforms, or changes in base level (Hack, 1965). Specifically, Duluth area longitudinal profiles reflect bedrock type and distribution of fault zones, variations in the texture of glacial deposits, glacial landforms (such 

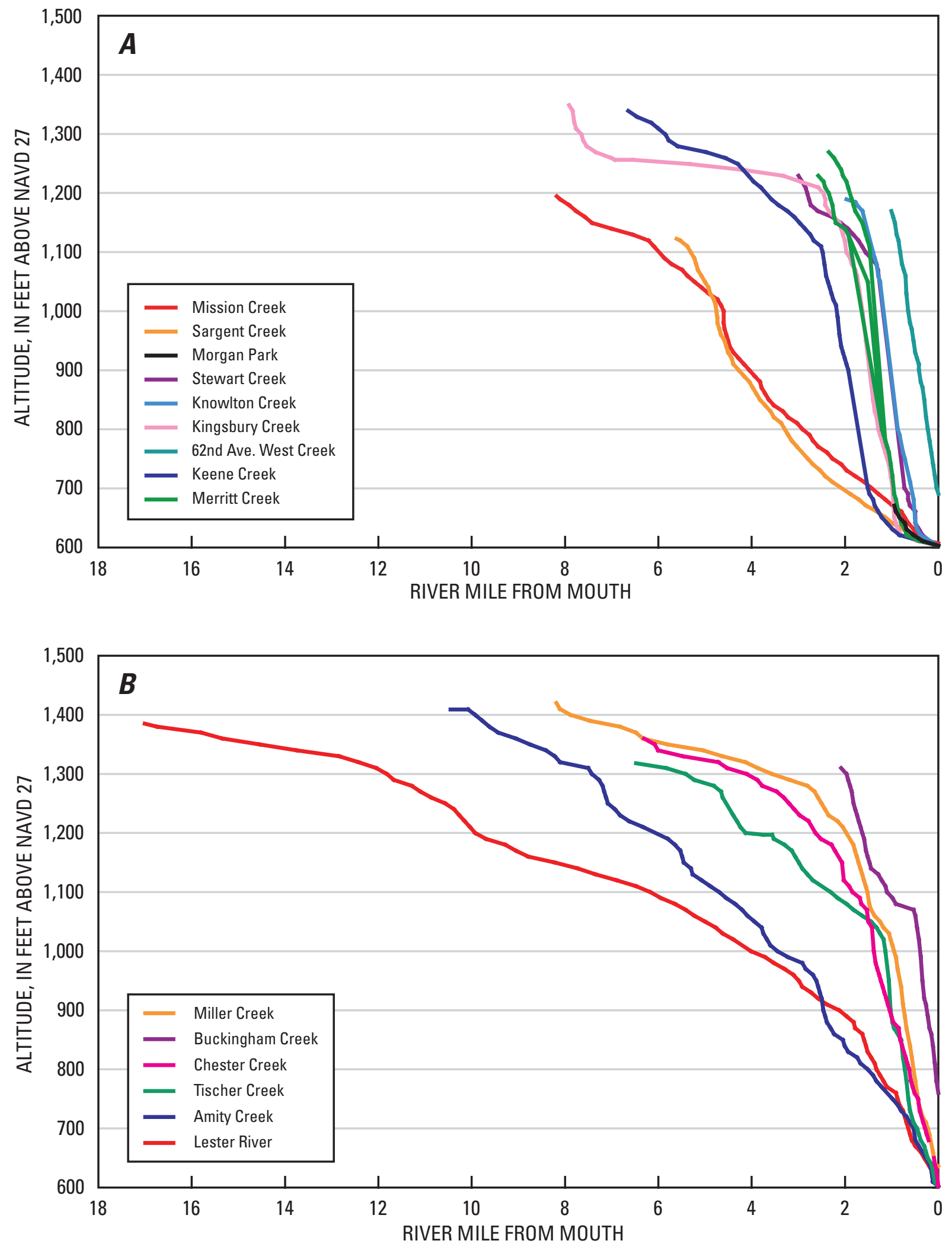

Figure 9. Longitudinal profiles of streams in the $\boldsymbol{A}$, southwestern, and $\boldsymbol{B}$, northeastern parts of the Duluth, Minn., area. 
Table 2. Conditions at the mouths of Duluth-area streams, Minn.

\begin{tabular}{ll}
\hline \multicolumn{1}{c}{ Stream } & \multicolumn{1}{c}{ Conditions } \\
\hline Mission Creek & Channel open to the St. Louis River \\
Sargent Creek & Channel open to the St. Louis River estuary \\
U.S. Steel Creek & Channel open to the St. Louis River estuary \\
Morgan Park Creek & Channel open to the St. Louis River estuary \\
Stewart Creek & Channel open to the St. Louis River estuary \\
Lenroot Creek & Channel open to the St. Louis River estuary \\
Knowlton Creek & Channel open to the St. Louis River estuary \\
Kingsbury Creek & Channel open to the St. Louis River estuary \\
62nd Ave. Creek & Runs underground by Grand Ave. \\
Keene Creek & Channel open to the St. Louis River estuary \\
Merritt Creek & Channel open to the St. Louis River estuary \\
Miller Creek & Runs underground through downtown Duluth \\
Buckingham Creek & Runs underground through downtown Duluth \\
Coffee Creek & Runs underground through downtown Duluth \\
Brewery and Greys Creeks & Runs underground through downtown Duluth \\
Chester Creek & Runs underground in downtown Duluth \\
Tischer Creek & Channel open to Lake Superior \\
34th to 50th Ave. Creeks & Some channels open, others run underground \\
Amity Creek & Channel open to Lake Superior \\
Lester River & Channel open to Lake Superior \\
\hline
\end{tabular}

as moraines and outwash plains), postglacial landforms from beach ridges, shorelines or melt-water valleys, and the present level of Lake Superior. Reaches with steep slopes are prone to long-term erosional conditions, whereas reaches with gentle slopes are prone to long-term depositional conditions (Knighton, 1998). Reaches in a transition from steep to gentle slopes may change from erosional to depositional if upstream inputs of water and sediment change.

The upper main stems tend to have gentle slopes for headwaters in glacial deposits at altitudes higher than about 1,200 ft. Irregularities in the longitudinal profiles are common from about 1,200 to $1,000 \mathrm{ft}$ because of the boundary among glacial deposits, bedrock, and glacial lake shorelines. The steep slopes are on middle and lower main stems and tributaries indicate where the streams intersect bedrock (fig. 4). Streams flowing over the Duluth Complex gabbro intrusions have the steepest slopes. Below 1,100 ft, longitudinal profiles for Mission Creek and Sargent Creek are concave upward and less steep than streams to the northeast because Mission and Sargent Creek intersect the less resistant Animikie sedimentary rocks and glacial lake plain. In the northeastern part of the city, Lester River, Amity Creek, and a portion of Tischer Creek have more gentle slopes than streams in the center of the city but steeper slopes than Mission and Sargent Creeks because the streams intersect Keweenawan volcanic rocks. Only a few streams in the southwestern part of the study area have enough length on till plain or built-out land to have a gentle slope near their mouths in the St. Louis River estuary (fig. 1).

\section{Segment Characteristics}

Streams with gentle slopes (less than 0.3 percent) generally were headwater streams flowing on glacial deposits with no valley development with wetland vegetation (lowland shrub or forest); whereas streams with moderate to steep slopes flowed on bedrock and had confined or entrenched valleys (tables 3 and 4). Alluvial valleys 
Table 3. Number of stream segments in each slope/drainage-network position category for Duluth-area streams, Minn.

$[<$, less than; $>$, greater than $]$

\begin{tabular}{cccccccc}
\hline $\begin{array}{c}\text { Slope category } \\
\text { (percent) }\end{array}$ & $\begin{array}{c}\text { Upper } \\
\text { main stem }\end{array}$ & $\begin{array}{c}\text { Middle } \\
\text { main stem }\end{array}$ & $\begin{array}{c}\text { Lower } \\
\text { main stem }\end{array}$ & $\begin{array}{c}\text { Upper } \\
\text { tributary }\end{array}$ & $\begin{array}{c}\text { Middle } \\
\text { tributary }\end{array}$ & $\begin{array}{c}\text { Lower } \\
\text { tributary }\end{array}$ & Total \\
\hline$<0.3$ & 4 & 0 & 0 & 3 & 0 & 0 & $\mathbf{7}$ \\
$0.3-1$ & 10 & 7 & 8 & 5 & 4 & 1 & $\mathbf{3 5}$ \\
$>1-2$ & 4 & 19 & 6 & 3 & 9 & 1 & $\mathbf{4 2}$ \\
$>2-4$ & 7 & 6 & 10 & 6 & 15 & 10 & $\mathbf{5 4}$ \\
$>4-8$ & 2 & 4 & 9 & 3 & 3 & 10 & $\mathbf{3 1}$ \\
$>8$ & 1 & 7 & 7 & 1 & 2 & 1 & $\mathbf{1 9}$ \\
Total & $\mathbf{2 8}$ & $\mathbf{4 3}$ & $\mathbf{4 0}$ & $\mathbf{2 1}$ & $\mathbf{3 3}$ & $\mathbf{2 3}$ & $\mathbf{1 8 8}$ \\
\hline
\end{tabular}

Table 4. Number of stream segments in each slope/valley type category for Duluth-area streams, Minn.

$[<$, less than; $>$, greater than $]$

\begin{tabular}{ccccccc}
\hline $\begin{array}{c}\text { Slope category } \\
\text { (percent) }\end{array}$ & No valley & $\begin{array}{c}\text { Confined } \\
\text { (origin not } \\
\text { determined) }\end{array}$ & Entrenched & Alluvial & $\begin{array}{c}\text { Bedrock } \\
\text { (confined) }\end{array}$ & Total \\
\hline$<0.3$ & 7 & 0 & 0 & 0 & 0 & $\mathbf{7}$ \\
$0.3-1$ & 26 & 1 & 4 & 4 & 0 & $\mathbf{3 5}$ \\
$>1-2$ & 17 & 10 & 12 & 0 & 3 & $\mathbf{4 2}$ \\
$>2-4$ & 1 & 37 & 10 & 1 & 5 & $\mathbf{5 4}$ \\
$>4-8$ & 0 & 15 & 8 & 0 & 8 & $\mathbf{3 1}$ \\
$>8$ & 0 & 0 & 9 & 0 & 10 & $\mathbf{1 9}$ \\
Total & $\mathbf{5 1}$ & $\mathbf{6 3}$ & $\mathbf{4 3}$ & $\mathbf{5}$ & $\mathbf{2 6}$ & $\mathbf{1 8 8}$ \\
\hline
\end{tabular}

were rare because of the small watershed sizes, bedrock control, and geologically young age of the streams (less than 10,000 years). Main-stem segments with moderate slopes typically were in the boundary zone between glacial deposits and bedrock. Tributaries to middle or lower main stems most commonly had $2-8$ percent slopes in confined valleys. The combination of steep slopes and confined or entrenched valleys are indicative of a high potential for landslides along valley sides, especially if sandy glacial lake shoreline deposits are present. If valley sides are made up of unconsolidated deposits, the potential for landslides, mass wasting, and channel erosion increases if runoff and flood peaks increase (Fitzpatrick and others, 1999).

Characteristics for all the segments are listed in table 5, with a list of abbreviations and definitions in table 6. Drainage-network position, slope, valley type, bedrock type, glacial landform, and dominant land cover are described in table 5 . The table has site identification numbers for segments with rapid assessment or intensive survey sites. Based on field reach-scale data and air photos, characteristics of channel type, dominant substrate, mode of sediment transport, flood attenuation, observed/ expected causes for disturbance, presence of storm drains, and potential for geomorphic change are described for segments with field sites and extrapolated for other segments. Almost all of the segments have one or more observed or expected causes for disturbance, usually land clearing, urban development, road drainage, and inputs from upstream segments. The only exception is six tributary segments near the middle/lower main stem of Mission Creek that have complete forest cover and no roads. 
Table 5. Geomorphic characteristics of stream segments for Duluth-area streams, Minn., 2003-2004.

[See table 6 for explanation of abbreviations. Stream segments are shown on figure 13. Data in italics are extrapolated from reach assessments of sites with the same segment category. Data in bold are rapid assessment or intensive survey sites. USGS, U.S. Geological Survey; ID, identification number; --, no detailed measurements taken; \%, percent; >, greater than; <, less than]

\begin{tabular}{|c|c|c|c|c|c|c|c|c|c|c|c|}
\hline $\begin{array}{l}\text { Segment } \\
\text { ID }\end{array}$ & Stream name & $\begin{array}{l}\text { Topographic } \\
\text { map }\end{array}$ & $\begin{array}{l}\text { USGS } \\
\text { reach } \\
\text { ID }\end{array}$ & $\begin{array}{c}\text { Drainage- } \\
\text { network } \\
\text { position }\end{array}$ & $\begin{array}{c}\text { Slope } \\
\%\end{array}$ & $\begin{array}{l}\text { Valley } \\
\text { type }\end{array}$ & $\begin{array}{l}\text { Bedrock } \\
\text { type }\end{array}$ & $\begin{array}{l}\text { Glacial } \\
\text { landform }\end{array}$ & $\begin{array}{l}\text { Dominant } \\
\text { land cover }\end{array}$ & $\begin{array}{l}\text { Inter- } \\
\text { mittent }\end{array}$ & $\begin{array}{c}\text { Channel } \\
\text { type }\end{array}$ \\
\hline b1 & Buckingham Creek & Duluth & -- & LM & $>8$ & $\mathrm{E}$ & GAB & BED & $\mathrm{U}$ & $\mathrm{N}$ & $\mathrm{BE}$ \\
\hline b2 & Buckingham Creek & Duluth Heights & 8 & MM & $>1-2$ & $\mathbf{N}$ & GAB & BED & $\mathbf{U}$ & $\mathbf{N}$ & PB \\
\hline b3 & Buckingham Creek & Duluth Heights & -- & UT & $>2-4$ & $\mathrm{C}$ & GAB & BED & F & $Y$ & $\mathrm{CO}$ \\
\hline c1 & Chester Creek & Duluth & 9 & LM & $>4-8$ & $\mathbf{E}$ & VOL & BED & $\mathbf{U}$ & $\mathbf{N}$ & BE \\
\hline c2 & Chester Creek & Duluth & 43 & MM & $>1-2$ & $\mathbf{N}$ & GAB & OW/BED & $\mathbf{U} / \mathbf{F}$ & $\mathbf{N}$ & PR/SP \\
\hline c3 & Chester Creek & $\begin{array}{l}\text { Duluth, Duluth } \\
\text { Heights }\end{array}$ & -- & UM & $0.3-1$ & $\mathrm{~N}$ & GAB & SD/BED & $\mathrm{S} / \mathrm{W}$ & $\mathrm{N}$ & WE \\
\hline $\mathrm{c} 4$ & Chester Creek & Duluth & -- & MM & $<0.3$ & $\mathrm{~N}$ & BUR/GAB & $O W$ & $\mathrm{U}$ & $\mathrm{N}$ & $\mathrm{AR}$ \\
\hline $\mathrm{c} 5$ & Chester Creek & $\begin{array}{l}\text { Duluth, Duluth } \\
\text { Heights }\end{array}$ & -- & UM & $>1-2$ & $\mathrm{~N}$ & GAB & SD & $\mathrm{S} / \mathrm{U}$ & $\mathrm{N}$ & WE \\
\hline c6 & Chester Creek & $\begin{array}{l}\text { Duluth, Duluth } \\
\text { Heights }\end{array}$ & -- & UM & $>1-2$ & $\mathrm{~N}$ & GAB & BED & $\mathrm{U}$ & $\mathrm{N}$ & $\mathrm{AR} / \mathrm{PB}$ \\
\hline c7 & Chester Creek & Duluth & -- & LT & $>2-4$ & $\mathrm{C}$ & VOL & BED & $\mathrm{F} / \mathrm{U}$ & $Y$ & $\mathrm{CO}$ \\
\hline $\mathrm{k} 1$ & Keene Creek & West Duluth & -- & LM & $0.3-1$ & $\mathrm{~N}$ & GAB & $\mathrm{TP}$ & $\mathrm{U}$ & $\mathrm{N}$ & AR \\
\hline k2 & Keene Creek & West Duluth & -- & LM & $>2-4$ & $\mathrm{C}$ & GAB & BED & $\mathrm{U}$ & $\mathrm{N}$ & $B E D / C A$ \\
\hline$k 2 a$ & Keene Creek & West Duluth & 42 & LM & $>1-2$ & $\mathbf{N}$ & GAB & TP & $\mathbf{U}$ & $\mathbf{N}$ & $\mathrm{PB} / \mathrm{AR}$ \\
\hline k3 & Keene Creek & $\begin{array}{l}\text { West Duluth, } \\
\text { Duluth Heights }\end{array}$ & -- & MM & $>4-8$ & $\mathrm{E}$ & GAB & BED & $\mathrm{U} / \mathrm{F}$ & $\mathrm{N}$ & $\mathrm{CA} / \mathrm{BE}$ \\
\hline k4 & Keene Creek & Duluth Heights & 6 & MM & $>1-2$ & $\mathbf{E}$ & GAB & BED/SH & $F / U$ & $\mathbf{N}$ & PR/SP \\
\hline $\mathrm{k} 5$ & Keene Creek & Duluth Heights & -- & UM & $0.3-1$ & $\mathrm{~N}$ & GAB & $\mathrm{SD} / \mathrm{BED}$ & F/U/W & $\mathrm{N}$ & $W E$ \\
\hline k6 & Keene Creek trib & Duluth Heights & 5 & MT & $>4-8$ & B & GAB & BED/SH & $\mathbf{U} / \mathbf{F}$ & $\mathbf{N}$ & $\mathrm{CA} / \mathrm{CO}$ \\
\hline $\operatorname{kg} 1$ & Kingsbury Creek & West Duluth & -- & LM & $0.3-1$ & A & BUR & $\mathrm{TP}$ & $\mathrm{U}$ & $\mathrm{N}$ & AR \\
\hline $\mathrm{kg} 2$ & Kingsbury Creek & West Duluth & -- & $\mathrm{LM}$ & $>4-8$ & $\mathrm{C}$ & GAB & $\mathrm{BED} / \mathrm{SH}$ & $\mathrm{U}$ & $\mathrm{N}$ & $\mathrm{BE}$ \\
\hline $\operatorname{kg} 2 \mathrm{a}$ & Kingsbury Creek & West Duluth & -- & LM & $>2-4$ & $\mathrm{C}$ & GAB & BED & $\mathrm{F} / \mathrm{U}$ & $\mathrm{N}$ & $\mathrm{BED} / \mathrm{CA}$ \\
\hline $\mathrm{kg} 2 \mathrm{~b}$ & Kingsbury Creek & West Duluth & -- & LM & $>4-8$ & $\mathrm{C}$ & GAB & BED & $\mathrm{U}$ & $\mathrm{N}$ & $\mathrm{BE}$ \\
\hline $\mathrm{kg} 2 \mathrm{c}$ & Kingsbury Creek & West Duluth & -- & LM & $>8$ & $\mathrm{C}$ & GAB & BED & $\mathrm{U}$ & $\mathrm{N}$ & $\mathrm{BE}$ \\
\hline $\operatorname{kg} 2 d$ & Kingsbury Creek & West Duluth & -- & LM & $>2-4$ & $\mathrm{C}$ & GAB & BED & $\mathrm{U}$ & $\mathrm{N}$ & $\mathrm{BED} / \mathrm{CA}$ \\
\hline kg3 & Kingsbury Creek & West Duluth & 41 & MM & $>2-4$ & B & GAB & $\begin{array}{r}\text { SH/SD/ } \\
\text { BED }\end{array}$ & $\mathbf{U} / \mathbf{F}$ & $\mathbf{N}$ & SP/CA \\
\hline $\mathrm{kg} 4$ & Kingsbury Creek & West Duluth & -- & MM & $0.3-1$ & $\mathrm{~N}$ & GAB & $\mathrm{SD}$ & $\mathrm{W} / \mathrm{U}$ & $\mathrm{N}$ & $\mathrm{AR}$ \\
\hline kg5 & Kingsbury Creek & Duluth Heights & -- & $\mathrm{UM}$ & $<0.3$ & $\mathrm{~N}$ & GAB & SD/BED & $\mathrm{W}$ & $\mathrm{N}$ & $\mathrm{AR} / \mathrm{WE}$ \\
\hline $\operatorname{kg} 6$ & Kingsbury Creek & Duluth Heights & -- & $\mathrm{UM}$ & $0.3-1$ & $\mathrm{~N}$ & GAB & $\mathrm{SD} / \mathrm{BED}$ & W & $\mathrm{N}$ & AR/DW \\
\hline $\mathrm{kg} 7$ & Kingsbury Creek & Duluth Heights & -- & UT & $>1-2$ & $\mathrm{~N}$ & GAB & SD/BED & W & $Y$ & $P R$ \\
\hline $\operatorname{kg} 8$ & Kingsbury Creek & Duluth Heights & -- & UT & $>4-8$ & $\mathrm{C}$ & GAB & $\mathrm{SD} / \mathrm{BED}$ & $\mathrm{F} / \mathrm{U}$ & $Y$ & $\mathrm{CO}$ \\
\hline kw1 & Knowlton Creek & West Duluth & -- & LM & $0.3-1$ & $\mathrm{~N}$ & GAB & $\mathrm{TP}$ & $\mathrm{F} / \mathrm{U}$ & $\mathrm{N}$ & $\mathrm{AR}$ \\
\hline kw2 & Knowlton Creek & West Duluth & 4 & MM & $>4-8$ & $\mathbf{E}$ & GAB & BED & $\mathbf{U}$ & $\mathbf{N}$ & BE/CA \\
\hline kw2a & Knowlton Creek & West Duluth & -- & LM & $>8$ & $\mathrm{E}$ & GAB & BED & $\mathrm{F}$ & $\mathrm{N}$ & $\mathrm{BE}$ \\
\hline $\mathrm{kw} 2 \mathrm{~b}$ & Knowlton Creek & West Duluth & -- & MM & $>4-8$ & $\mathrm{E}$ & GAB & BED & $\mathrm{U} / \mathrm{F}$ & $\mathrm{N}$ & $\mathrm{BE} / \mathrm{CA}$ \\
\hline $\mathrm{kw} 2 \mathrm{c}$ & Knowlton Creek & West Duluth & -- & MM & $>8$ & E & GAB & $\mathrm{BED} / \mathrm{SH}$ & $\mathrm{S} / \mathrm{U}$ & $\mathrm{N}$ & $\mathrm{BE}$ \\
\hline kw3 & Knowlton Creek & West Duluth & -- & UM & $>1-2$ & $\mathrm{~N}$ & GAB & $\mathrm{SD}$ & $\mathrm{U}$ & $\mathrm{N}$ & $\mathrm{AR}$ \\
\hline 11 & Lester River & $\begin{array}{l}\text { Duluth, Lake- } \\
\text { wood }\end{array}$ & 19,31 & $\mathbf{L M}$ & $>2-4$ & $\mathbf{E}$ & VOL & BED & $\mathbf{F}$ & $\mathbf{N}$ & BE \\
\hline
\end{tabular}




\begin{tabular}{|c|c|c|c|c|c|c|c|c|}
\hline $\begin{array}{l}\text { Dominant } \\
\text { substrate }\end{array}$ & $\begin{array}{c}\text { Mode of } \\
\text { sediment transport }\end{array}$ & $\begin{array}{c}\text { Flood } \\
\text { attenuation }\end{array}$ & $\begin{array}{c}\text { Dominant } \\
\text { geomorphic } \\
\text { processes }\end{array}$ & $\begin{array}{c}\text { Observed/expected } \\
\text { causes for } \\
\text { disturbance }\end{array}$ & $\begin{array}{c}\text { Storm- } \\
\text { drain } \\
\text { outfalls }\end{array}$ & $\begin{array}{c}\text { Potential } \\
\text { geomorphic } \\
\text { changes }\end{array}$ & $\begin{array}{c}\begin{array}{c}\text { Sensitivity } \\
\text { to } \\
\text { disturbance }\end{array} \\
\end{array}$ & $\begin{array}{c}\text { Geomorphic } \\
\text { segment } \\
\text { category }\end{array}$ \\
\hline $\mathrm{BE}$ & $\mathrm{T}$, SIp & NO & EX & UP, HI, UR & Y & $\mathrm{Y}$ & LO & B \\
\hline GV & SIa & FP & $\mathbf{A}$ & UP, CA, UR, GV, CL & $\mathbf{N}$ & $\mathbf{Y}$ & MOD & M1 \\
\hline $\mathrm{CO} / \mathrm{BO}$ & SOi, SOw & NO & $\mathrm{I}, \mathrm{W}$ & $\mathrm{CL}, \mathrm{CA}, \mathrm{RO}, \mathrm{GV}$ & $\mathrm{N}$ & $\mathrm{Y}$ & MOD & $\mathrm{T} 2$ \\
\hline BO/BE & $\mathrm{T}, \mathrm{SOb}$ & NO & EX, EBL & UP, HI, UR & $\mathbf{Y}$ & $\mathbf{Y}$ & LO & B \\
\hline GV & $\begin{array}{l}\text { SOi, SOw, T, SIc, } \\
\text { SIp }\end{array}$ & $\mathbf{F P}$ & I, EBA, W, LM, BF & UP, UR, RO, CL & $\mathbf{Y}$ & $\mathbf{Y}$ & HI & M1 \\
\hline$G V$ & SOw, SOi, T, SIa & WE & I, W, EBA, A & $\mathrm{CL}, \mathrm{UR}, \mathrm{RO}, \mathrm{CA}$ & $\mathrm{N}$ & $\mathrm{Y}$ & MOD & W.3 \\
\hline FINES & $\mathrm{T}, S O w$ & NO & EBA, LM, $W, \mathrm{~A}$ & $\mathrm{UR}, \mathrm{CA}, \mathrm{RO}, \mathrm{CL}$ & $\mathrm{Y}$ & $\mathrm{Y}$ & MOD & $\mathrm{W}$ \\
\hline$G V$ & SOw, SOi & $F P$ & I, W, EBA & CL, UR, RO & $\mathrm{Y}$ & $\mathrm{Y}$ & $\mathrm{HI}$ & $\mathrm{U} 1$ \\
\hline $\mathrm{CO}$ & SOb, SOi, SOw, T & NO & I, W, EBA, LM & $\mathrm{UR}, \mathrm{RO}, \mathrm{CA}, \mathrm{CL}$ & $\mathrm{Y}$ & $\mathrm{Y}$ & $\mathrm{HI}$ & $\mathrm{U} 1$ \\
\hline$B O$ & $\mathrm{SO}$, SOb, SOw & NO & I, W, EBA, EBL, EX & UR, RO, CL & $\mathrm{Y}$ & $\mathrm{Y}$ & MOD & LT \\
\hline$B O$ & $\mathrm{~T}$, SIa & NO & $A$ & UP, UR, RO, CA & $\mathrm{N}$ & $\mathrm{Y}$ & LO & A \\
\hline$B O$ & SOb, SOw, T & NO & W, EBA, EBL, EX & UP, RO, UR & $\mathrm{Y}$ & $\mathrm{Y}$ & MOD & L2 \\
\hline BO & $\mathbf{T}$ & NO & ST & UP, UR, RO, CA & $\mathbf{Y}$ & $\mathbf{Y}$ & LO & L1 \\
\hline BO & $\mathrm{T}, \mathrm{SOw}, \mathrm{SOb}$ & NO & W, EX, EBL, EBA & UR, RO, UP, GV, CL & $\mathrm{Y}$ & $\mathrm{Y}$ & MOD & B \\
\hline $\mathrm{BO} / \mathrm{CO}$ & T, SOw, SOi & NO & $\mathrm{W}, \mathbf{E B A}, I, L M$ & UP, GV, UR, RO, CL & $\mathbf{N}$ & $\mathbf{Y}$ & HI & M1 \\
\hline SA/FINES & SOw, SOi, SIa & WE & W, EBA, I, $A$ & UR, RO, GV & $\mathrm{N}$ & $\mathrm{Y}$ & MOD & W. 3 \\
\hline BO & $\mathbf{T}$ & NO & $\mathbf{E X}$ & UR, RO, GV & $\mathbf{N} ?$ & $\mathbf{Y}$ & MOD & BT \\
\hline BO & T, SIa & NO & $S T, A$ & UP, RO, CL & $\mathrm{Y}$ & $\mathrm{Y}$ & LO & A \\
\hline $\mathrm{BE}$ & SOw, SOi, SIc, T & NO & W, EBA, EX, BF & UR, RO, UP & $\mathrm{Y}$ & Y & MOD & B \\
\hline$B O / B E$ & SOw, T & NO & W, EBA, EX, BF & UP, RO, UR & $\mathrm{N}$ & $\mathrm{Y}$ & LO & L2 \\
\hline $\mathrm{BE}$ & SOw, T & NO & W, EBA, EX, BF & UR, RO, UP & $\mathrm{N}$ & $\mathrm{Y}$ & LO & B \\
\hline $\mathrm{BE}$ & $\mathrm{SOb}, \mathrm{SOw}, \mathrm{T}$ & NO & EX & UP, UR, RO & $\mathrm{Y}$ & $\mathrm{Y}$ & LO & B \\
\hline$B O$ & $S O b, \mathrm{~T}, \mathrm{SOw}, \mathrm{SIc}$ & $\mathrm{NO}$ & EX, EBA, W, BF & UP, UR, RO & $\mathrm{Y}$ & $\mathrm{Y}$ & MOD & L2 \\
\hline BO & SOb, SOw, T, SIc & NO & $\begin{array}{l}\text { EBL, EBA, LM, } \\
\text { BF, W }\end{array}$ & UP, RO, UR & $\mathbf{N}$ & $\mathbf{Y}$ & HI & M2 \\
\hline SA/FINES & SOb, T, SOw, SIc & WE & EBA, I, $W, \mathrm{~A}$ & UP, CA, UR, RO, CL & $\mathrm{N}$ & $\mathrm{Y}$ & MOD & M.3 \\
\hline FINES & SOw, T, SIa & WE & EBA, LM, $I$, A & $\mathrm{CA}, \mathrm{RO}, \mathrm{CL}$ & $\mathrm{N}$ & $\mathrm{Y}$ & MOD & $\mathrm{W}$ \\
\hline SA/FINES & SOw, SIa, T & WE & $\mathrm{A}, I, W$ & $\mathrm{UP}, \mathrm{RO}, \mathrm{CA}, \mathrm{CL}$ & $\mathrm{N}$ & $\mathrm{Y}$ & LO & W.3 \\
\hline $\mathrm{CO}$ & SOw, SOi & NO & I & CL, UR, RO & $\mathrm{N}$ & $\mathrm{Y}$ & MOD/HI & $\mathrm{T} 1$ \\
\hline$B O$ & SOw, SOi & $\mathrm{NO}$ & I & $\mathrm{UR}, \mathrm{RO}, \mathrm{CL}$ & $\mathrm{N}$ & $\mathrm{Y}$ & $\mathrm{MOD} / \mathrm{HI}$ & BT \\
\hline SA/FINES & T, SIa, SOb & FP & A & UP, CA, RO & $\mathrm{Y}$ & $\mathrm{Y}$ & MOD & A \\
\hline BO & $\mathrm{T}, \mathrm{SO} w, \mathrm{SOb}$ & NO & EX, EBL, EBA & UP, RO, UR, CA & $\mathbf{N}$ & $\mathbf{Y}$ & MOD & B \\
\hline $\mathrm{BE}$ & $\mathrm{T}$ & $\mathrm{NO}$ & EX & UP, RO, UR & $\mathrm{N}$ & $\mathrm{Y}$ & LO & B \\
\hline $\mathrm{BO}$ & $\mathrm{T}$ & NO & EX & UP, RO, UR & $\mathrm{N}$ & $\mathrm{Y}$ & LO & B \\
\hline $\mathrm{BE}$ & $\mathrm{T}$ & $\mathrm{NO}$ & EX & UP, RO, UR & $\mathrm{N}$ & $\mathrm{Y}$ & LO & B \\
\hline$P B$ & $\mathrm{~T}, \mathrm{SOw}$ & NO & A & CA, RO, UR & $\mathrm{N}$ & $\mathrm{Y}$ & $\mathrm{HI}$ & U1 \\
\hline BE & T, SIp & NO & EX, W, BF, EBL & UP, HI & $\mathbf{Y}$ & $\mathbf{Y}$ & LO & L2 \\
\hline
\end{tabular}


Table 5. Geomorphic characteristics of stream segments for Duluth-area streams, Minn., 2003-2004—Continued

[See table 6 for explanation of abbreviations. Stream segments are shown on figure 13. Data in italics are extrapolated from reach assessments of sites with the same segment category. Data in bold are rapid assessment or intensive survey sites. USGS, U.S. Geological Survey; ID, identification number; --, no detailed measurements taken; \%, percent; >, greater than; <, less than]

\begin{tabular}{|c|c|c|c|c|c|c|c|c|c|c|c|}
\hline $\begin{array}{l}\text { Segment } \\
\text { ID }\end{array}$ & Stream name & $\begin{array}{l}\text { Topographic } \\
\text { map }\end{array}$ & $\begin{array}{l}\text { USGS } \\
\text { reach } \\
\text { ID }\end{array}$ & $\begin{array}{c}\text { Drainage- } \\
\text { network } \\
\text { position }\end{array}$ & $\begin{array}{c}\text { Slope } \\
\%\end{array}$ & $\begin{array}{c}\text { Valley } \\
\text { type }\end{array}$ & $\begin{array}{c}\text { Bedrock } \\
\text { type }\end{array}$ & $\begin{array}{l}\text { Glacial } \\
\text { landform }\end{array}$ & $\begin{array}{l}\text { Dominant } \\
\text { land cover }\end{array}$ & $\begin{array}{l}\text { Inter- } \\
\text { mittent }\end{array}$ & $\begin{array}{c}\text { Channel } \\
\text { type }\end{array}$ \\
\hline 110 & Amity Creek & Arnold, Duluth & -- & MM & $0.3-1$ & $\mathrm{~N}$ & GAB & SD & $\mathrm{A} / \mathrm{F}$ & $\mathrm{N}$ & $P R$ \\
\hline 111 & Amity Creek & Arnold, Duluth & -- & MT & $>1-2$ & $\mathrm{~N}$ & GAB & SD & $\mathrm{AF}$ & $\mathrm{N}$ & $P R$ \\
\hline 112 & Amity Creek & Arnold & -- & MM & $>1-2$ & $\mathrm{~N}$ & BUR/GAB & SD & $\mathrm{W}$ & $\mathrm{N}$ & $P R$ \\
\hline 113 & Amity Creek & Arnold & -- & UM & $<0.3$ & $\mathrm{~N}$ & BUR/GAB & SD & $\mathrm{W}$ & $\mathrm{N}$ & $W E$ \\
\hline 114 & Amity Creek & Arnold & -- & UM & $0.3-1$ & $\mathrm{~N}$ & BUR/GAB & SD & WF & $\mathrm{N}$ & $W E / P R$ \\
\hline 115 & Amity Creek & Arnold & -- & UT & $>1-2$ & $\mathrm{C}$ & BUR/GAB & SD & $\mathrm{F}$ & $\mathrm{N}$ & $P R$ \\
\hline $116 \mathrm{a}$ & Amity Creek & Arnold & -- & UM & $>1-2$ & $\mathrm{~N}$ & BUR/GAB & SD & F/A/W & $\mathrm{N}$ & $P R$ \\
\hline $116 \mathrm{~b}$ & Lester River & $\begin{array}{l}\text { French River, } \\
\text { Arnold }\end{array}$ & -- & MT & $>2-4$ & $\mathrm{C}$ & VOL/IN & SD & F/A & $\mathrm{N}$ & $C A$ \\
\hline 117 & Amity Creek & Arnold & -- & UT & $<0.3$ & $\mathrm{~N}$ & BUR/GAB & SD & W & $\mathrm{N}$ & WE \\
\hline 118 & Amity Creek & Arnold & -- & UT & $>1-2$ & $\mathrm{~N}$ & BUR/GAB & SD & F/W & $Y$ & $P R$ \\
\hline 119 & Lester River & French River & -- & MM & $0.3-1$ & $\mathrm{E}$ & IN & $\mathrm{TP}$ & F/A & $\mathrm{N}$ & PR \\
\hline 119a & Lester River & French River & 20 & MM & $>1-2$ & $\mathbf{E}$ & VOL/IN & BED & F/A & $\mathbf{N}$ & PB/SP \\
\hline 12 & Amity Creek & Duluth & 11 & $\mathbf{L M}$ & $>2-4$ & $\mathbf{E}$ & VOL & BED & $F / U$ & $\mathbf{N}$ & BE \\
\hline 120 & Lester River & $\begin{array}{l}\text { French River, } \\
\text { Arnold }\end{array}$ & 23 & MM & $0.3-1$ & $\mathbf{E}$ & BUR/IN & SH & $\mathbf{F}$ & $\mathbf{N}$ & PR \\
\hline 121 & Lester River & French River & 21 & MM & $>2-4$ & B & IN & BED/SH & F/A & $\mathbf{N}$ & PB \\
\hline 122 & Lester River & Arnold & 22 & MM & $>1-2$ & $\mathbf{N}$ & BUR/IN & SD/SH & $\mathbf{F}$ & $\mathbf{N}$ & PB \\
\hline 123 & Lester River & Arnold & -- & UT & $0.3-1$ & $\mathrm{~N}$ & BUR/IN & $\mathrm{SD}$ & W & $\mathrm{N}$ & WE \\
\hline 124 & Lester River & Arnold & -- & MT & $>2-4$ & $\mathrm{C}$ & BUR/IN & SD & F/A & $\mathrm{N}$ & $P B$ \\
\hline $125 \mathrm{a}$ & Lester River & Arnold & -- & MT & $0.3-1$ & $\mathrm{~N}$ & BUR/IN & $\mathrm{SD}$ & $\mathrm{W}$ & $\mathrm{N}$ & $W E / P R$ \\
\hline $125 \mathrm{~b}$ & Lester River & Arnold & -- & MT & $>1-2$ & $\mathrm{~N}$ & BUR/IN & IC & F/A & $\mathrm{N}$ & $P B$ \\
\hline 126 & Lester River & Arnold & -- & MT & $0.3-1$ & $\mathrm{~N}$ & BUR/IN & UN & F/A & $\mathrm{N}$ & AR/PR \\
\hline 127 & Lester River & Arnold & -- & MT & $0.3-1$ & $\mathrm{~N}$ & BUR/GAB & OW/SD & FW & $\mathrm{N}$ & WE \\
\hline 128 & Lester River & Arnold & 30 & MM & $>1-2$ & $\mathbf{E}$ & IN & IC & $\mathbf{A F}$ & $\mathbf{N}$ & PB \\
\hline 129 & Lester River & Arnold & -- & MT & $>1-2$ & $\mathrm{~N}$ & IN & $\mathrm{SD}$ & $\mathrm{W}$ & $\mathrm{N}$ & WE \\
\hline 13 & Amity Creek & Duluth & -- & LM & $>1-2$ & $\mathrm{C}$ & IN & $\begin{array}{c}\mathrm{BED} / \mathrm{SD} / \\
\mathrm{SH}\end{array}$ & $\mathrm{F}$ & $\mathrm{N}$ & $C A$ \\
\hline 130 & Lester River & Arnold & -- & MT & $0.3-1$ & $\mathrm{~N}$ & IN & $\mathrm{SD}$ & $\mathrm{W}$ & $\mathrm{N}$ & WE \\
\hline 131 & Lester River & Arnold & -- & MT & $0.3-1$ & $\mathrm{~N}$ & IN & $\mathrm{SD}$ & $\mathrm{W}$ & $\mathrm{N}$ & WE \\
\hline 132 & Lester River & Arnold & 24 & UM & $0.3-1$ & $\mathbf{N}$ & BUR/GAB & OW & $\mathrm{W} / \mathrm{F}$ & $\mathbf{N}$ & PR/PB \\
\hline 133 & Lester River & Arnold & & UT & $0.3-1$ & $\mathrm{~N}$ & BUR/GAB & $\mathrm{SD}$ & $\mathrm{W} / \mathrm{F}$ & $\mathrm{N}$ & WE \\
\hline 134 & Lester River & Arnold & $\begin{array}{c}25,26 \\
27,28 \\
29\end{array}$ & UM & $<0.3$ & $\mathbf{N}$ & BUR/GAB & SD & $\mathbf{W} / \mathbf{F}$ & $\mathbf{N}$ & WE \\
\hline 135 & Lester River & Arnold & -- & UT & $<0.3$ & $\mathrm{~N}$ & BUR/GAB & SD & $\mathrm{W}$ & $\mathrm{N}$ & WE \\
\hline 136 & Lester River & Arnold & -- & UT & $<0.3$ & $\mathrm{~N}$ & BUR/GAB & $\mathrm{SD}$ & $\mathrm{W}$ & $\mathrm{N}$ & WE \\
\hline 137 & Lester River & Duluth & -- & $\mathrm{LT}$ & $>4-8$ & $\mathrm{C} / \mathrm{E}$ & IN & BED & $\mathrm{F}$ & $\mathrm{Y}$ & $C O / B E$ \\
\hline 138 & Amity Creek & Duluth & -- & LT & $>2-4$ & $\mathrm{C}$ & IN & SD & F/A & Y & $\mathrm{CO}$ \\
\hline 139 & Amity Creek & Duluth & -- & $\mathrm{LT}$ & $>2-4$ & $\mathrm{C}$ & IN & SD & $\mathrm{F} / \mathrm{U}$ & $\mathrm{Y}$ & $\mathrm{CO}$ \\
\hline
\end{tabular}




\begin{tabular}{|c|c|c|c|c|c|c|c|c|}
\hline $\begin{array}{l}\text { Dominant } \\
\text { substrate }\end{array}$ & $\begin{array}{c}\text { Mode of } \\
\text { sediment transport }\end{array}$ & $\begin{array}{c}\text { Flood } \\
\text { attenuation }\end{array}$ & $\begin{array}{l}\text { Dominant } \\
\text { geomorphic } \\
\text { processes }\end{array}$ & $\begin{array}{c}\text { Observed/expected } \\
\text { causes for } \\
\text { disturbance }\end{array}$ & $\begin{array}{l}\text { Storm- } \\
\text { drain } \\
\text { outfalls }\end{array}$ & $\begin{array}{l}\text { Potential } \\
\text { geomorphic } \\
\text { changes }\end{array}$ & $\begin{array}{c}\text { Sensitivity } \\
\text { to } \\
\text { disturbance }\end{array}$ & $\begin{array}{c}\text { Geomorphic } \\
\text { segment } \\
\text { category }\end{array}$ \\
\hline$G V$ & $\mathrm{SOb}, \mathrm{SIc}$ & FP & EBA, W, LM, OS, BF & UP & $\mathrm{N}$ & $\mathrm{Y}$ & MOD/HI & M.3 \\
\hline$G V$ & $\mathrm{SOb}$ & FP & EBA, W, LM, OS, BF & $\mathrm{CL}$ & $\mathrm{N}$ & $\mathrm{Y}$ & MOD/HI & $\mathrm{T} 1$ \\
\hline$G V$ & Sob, SIb & WE & EBA, W, LM, OS & UP & $\mathrm{N}$ & $\mathrm{Y}$ & MOD & M1 \\
\hline FINES & SIa & WE & A & $\mathrm{CA}$ & $\mathrm{N}$ & $\mathrm{Y}$ & LO & W \\
\hline FINES & SIa & WE & A & UP, CA, CL & $\mathrm{N}$ & $\mathrm{Y}$ & LO & W.3 \\
\hline$G V$ & SOi, SOw & NO & I, EBA & $\mathrm{CL}, \mathrm{RO}$ & $\mathrm{N}$ & $\mathrm{Y}$ & MOD & $\mathrm{T} 1$ \\
\hline$G V$ & SOi, SOw & FP/WE & I, EBA & $\mathrm{RO}, \mathrm{UR}, \mathrm{CL}$ & $\mathrm{N}$ & $\mathrm{Y}$ & $\mathrm{HI}$ & U1 \\
\hline BO & SOi, SOw, SOb & NO & $\begin{array}{l}\text { I, EBA, EBL, W, LM, } \\
\text { BF }\end{array}$ & CL, UR, RO & $\mathrm{N}$ & $\mathrm{Y}$ & $\mathrm{HI}$ & $\mathrm{T} 2$ \\
\hline FINES & SIa & NO & I, EBA & UP, CA & $\mathrm{N}$ & Y & LO & W \\
\hline GV & SOi, SOw & WE & A & $\mathrm{CL}$ & $\mathrm{N}$ & $\mathrm{Y}$ & MOD & $\mathrm{T} 1$ \\
\hline $\mathrm{CO} / \mathrm{GV}$ & T, SOb, SOw, SIc & NO & $\begin{array}{l}\text { EBA, EBL, W, LM, } \\
\quad \text { BF }\end{array}$ & UP, CL, RO & $\mathrm{N}$ & $\mathrm{Y}$ & MOD & M.3 \\
\hline $\mathrm{CO} / \mathrm{BO}$ & T, SOb, SOw & NO & $\begin{array}{l}\text { EBA, EBL, W, LM, } \\
\text { BF }\end{array}$ & UP, CL, RO & $\mathbf{N}$ & $\mathbf{Y}$ & MOD & M1 \\
\hline $\mathbf{B E}$ & T, SIp & NO & $\mathbf{E X}, \mathbf{W}$ & UP, HI & $\mathbf{Y}$ & $\mathbf{Y}$ & LO & $\mathbf{L 2}$ \\
\hline GV & T, SOb, SOw & NO & W, EBA, EBL & UP & $\mathbf{N}$ & $\mathbf{Y}$ & HI & M.3 \\
\hline GV & T, SOb & NO & W, EX, EBA & UP, CL, UR, RO & $\mathbf{N}$ & $\mathbf{Y}$ & MOD & M2 \\
\hline GV & T, SIa & WE & $\mathbf{A}$ & UP, CL, UR, RO & $\mathbf{N}$ & $\mathbf{Y}$ & MOD & M1 \\
\hline FINES & SIa, SIb & WE & A & $\mathrm{CL}, \mathrm{RO}$ & $\mathrm{N}$ & $\mathrm{Y}$ & LO & W.3 \\
\hline$B O$ & SOw, SOi & NO & I, EBA, W & UP, CL, UR & $\mathrm{N}$ & $\mathrm{Y}$ & MOD & M2 \\
\hline FINES & SOw, SIa & NO & A & CL, CA, UR & $\mathrm{N}$ & $\mathrm{Y}$ & LO & W.3 \\
\hline$G V$ & T, SOw & NO & EBA, W, LM, OS & CL, GV & $\mathrm{N}$ & $\mathrm{Y}$ & MOD & $\mathrm{T} 1$ \\
\hline GV/FINES & T, SOw, SIa & WE & W, EBA & $\mathrm{CL}, \mathrm{RO}, \mathrm{CA}, \mathrm{GV}$ & $\mathrm{N}$ & $\mathrm{Y}$ & MOD & W. 3 \\
\hline FINES & SIa & WE & A & $\mathrm{CL}$ & $\mathrm{N}$ & $\mathrm{Y}$ & LO & W.3 \\
\hline $\mathrm{CO} / \mathrm{BO}$ & SOw, SOb, T & NO & W, EBA, EBL & UP, CL & $\mathbf{N}$ & $\mathbf{Y}$ & HI & M1 \\
\hline FINES & SIa & WE & A & $\mathrm{CL}$ & $\mathrm{N}$ & $\mathrm{Y}$ & LO & $\mathrm{T} 1$ \\
\hline BO & $T, S I c$ & NO & EX, W, EBL, LM, BF & UP & $\mathrm{N}$ & $\mathrm{Y}$ & MOD/HI & $\mathrm{L} 1$ \\
\hline FINES & SIa & WE & A & UP, CL, CA & $\mathrm{N}$ & $\mathrm{Y}$ & LO & W.3 \\
\hline FINES & SIa & WE & A & $\mathrm{CL}, \mathrm{RO}$ & $\mathrm{N}$ & $\mathrm{Y}$ & LO & W.3 \\
\hline $\mathrm{CO}$ & SIa & WE & ST & UP, GV, CL & $\mathbf{N}$ & $\mathbf{Y}$ & MOD & W.3 \\
\hline FINES & SIa & WE & $\mathrm{ST}$ & $\mathrm{RO}, \mathrm{UR}, \mathrm{CL}$ & $\mathrm{N}$ & $\mathrm{Y}$ & MOD & W.3 \\
\hline FINES & SOi, SIa & WE & I, W, EBA, A & RO, UR, CL & $\mathbf{N}$ & $\mathbf{Y}$ & LO & W \\
\hline FINES & SIa & WE & I, W, EBA, A & UR & $\mathrm{N}$ & $\mathrm{Y}$ & LO & W \\
\hline FINES & SOi, SIa & WE & I, W, EBA, A & UR, GV & $\mathrm{N}$ & $\mathrm{Y}$ & LO & W \\
\hline$B O / B E$ & $\mathrm{~T}, \mathrm{SOw}$ & NO & W, EX & CL, UR, RO & $\mathrm{N}$ & $\mathrm{Y}$ & MOD & BT \\
\hline $\mathrm{CO}$ & SOi, SOw, SOb & NO & I, EBL, EBA & $\mathrm{CL}$ & $\mathrm{N}$ & $\mathrm{Y}$ & $\mathrm{HI}$ & LT \\
\hline $\mathrm{CO}$ & SOi, SOw, SOb & NO & I, EBL, EBA & $\mathrm{CL}$ & $\mathrm{N}$ & $\mathrm{Y}$ & $\mathrm{HI}$ & LT \\
\hline
\end{tabular}


Table 5. Geomorphic characteristics of stream segments for Duluth-area streams, Minn., 2003-2004—Continued

[See table 6 for explanation of abbreviations. Stream segments are shown on figure 13. Data in italics are extrapolated from reach assessments of sites with the same segment category. Data in bold are rapid assessment or intensive survey sites. USGS, U.S. Geological Survey; ID, identification number; --, no detailed measurements taken; \%, percent; >, greater than; <, less than]

\begin{tabular}{|c|c|c|c|c|c|c|c|c|c|c|c|}
\hline $\begin{array}{l}\text { Segment } \\
\text { ID }\end{array}$ & Stream name & $\begin{array}{l}\text { Topographic } \\
\text { map }\end{array}$ & $\begin{array}{c}\text { USGS } \\
\text { reach } \\
\text { ID }\end{array}$ & $\begin{array}{c}\text { Drainage- } \\
\text { network } \\
\text { position }\end{array}$ & $\begin{array}{c}\text { Slope } \\
\%\end{array}$ & $\begin{array}{l}\text { Valley } \\
\text { type }\end{array}$ & $\begin{array}{c}\text { Bedrock } \\
\text { type }\end{array}$ & $\begin{array}{l}\text { Glacial } \\
\text { landform }\end{array}$ & $\begin{array}{l}\text { Dominant } \\
\text { land cover }\end{array}$ & $\begin{array}{l}\text { Inter- } \\
\text { mittent }\end{array}$ & $\begin{array}{c}\text { Channel } \\
\text { type }\end{array}$ \\
\hline $13 \mathbf{a}$ & Amity Creek & Duluth & $\begin{array}{c}12,13 \\
14,15 \\
16\end{array}$ & LM & $>1-2$ & $\mathbf{E}$ & VOL & BED & $\mathbf{F}$ & $\mathbf{N}$ & $\mathrm{PB} / \mathrm{BE}$ \\
\hline L3b & Amity Creek & Duluth & 17,18 & $\mathbf{L M}$ & $>2-4$ & $\mathbf{E}$ & IN & BED & $\mathbf{F}$ & $\mathbf{N}$ & BE \\
\hline 14 & Amity Creek & Duluth & -- & MM & $>1-2$ & $\mathrm{C}$ & IN & $\mathrm{BED} / \mathrm{SD}$ & F/A & $\mathrm{N}$ & $C A$ \\
\hline 140 & Amity Creek & Duluth & -- & LT & $>4-8$ & $\mathrm{C}$ & IN & SD & $\mathrm{F} / \mathrm{A} / \mathrm{U}$ & Y & $\mathrm{CO}$ \\
\hline 141 & Amity Creek & Duluth & -- & LT & $>4-8$ & $\mathrm{C}$ & IN & SD & F/A & $\mathrm{Y}$ & $\mathrm{CO}$ \\
\hline 142 & Amity Creek & Duluth & -- & LT & $>4-8$ & $\mathrm{C}$ & VOL & $\mathrm{BED} / \mathrm{TP}$ & $\mathrm{F} / \mathrm{U}$ & Y & $\mathrm{CO}$ \\
\hline 143 & Amity Creek & Duluth & -- & LT & $>4-8$ & $\mathrm{C}$ & VOL & BED/TP & $\mathrm{U} / \mathrm{F}$ & $\mathrm{Y}$ & $\mathrm{CO}$ \\
\hline 144 & Amity Creek & Duluth & -- & LT & $>2-4$ & $\mathrm{C}$ & IN & $\mathrm{SD} / \mathrm{SH}$ & A & Y & $\mathrm{CO}$ \\
\hline 145 & Amity Creek & Duluth & -- & LT & $>2-4$ & $\mathrm{C}$ & IN & $\begin{array}{c}\mathrm{BED} / \mathrm{SD} / \\
\mathrm{SH}\end{array}$ & A & Y & $\mathrm{CO}$ \\
\hline 146 & Amity Creek & Duluth & -- & LT & $>4-8$ & $\mathrm{C}$ & IN & $\begin{array}{c}\mathrm{BED} / \mathrm{SD} / \\
\mathrm{SH}\end{array}$ & $\mathrm{A} / \mathrm{F}$ & Y & $\mathrm{CO}$ \\
\hline 147 & Amity Creek & Duluth & -- & $\mathrm{LT}$ & $>4-8$ & $\mathrm{C}$ & IN & BED & $\mathrm{F}$ & $\mathrm{N}$ & $C O / C A$ \\
\hline 148 & Lester River & Duluth & -- & LT & $>2-4$ & $\mathrm{C}$ & VOL & $\mathrm{BED} / \mathrm{TP}$ & F/A & Y & $\mathrm{CO}$ \\
\hline 149 & Lester River & Lakewood, Duluth & -- & LT & $>4-8$ & $\mathrm{C}$ & VOL & $\mathrm{BED} / \mathrm{SH}$ & $\mathrm{A} / \mathrm{F}$ & $\mathrm{Y}$ & $\mathrm{CO}$ \\
\hline 15 & Amity Creek & Duluth & -- & MM & $0.3-1$ & $\mathrm{E}$ & IN & $\mathrm{BED} / \mathrm{SH}$ & $\mathrm{F} / \mathrm{U}$ & $\mathrm{N}$ & $P R$ \\
\hline 16 & Amity Creek & Duluth & -- & MM & $>1-2$ & $\mathrm{C}$ & GAB & BED & $\mathrm{F}$ & $\mathrm{N}$ & $P B$ \\
\hline 17 & Amity Creek & Duluth & -- & MM & $>2-4$ & $\mathrm{C}$ & GAB & BED & $\mathrm{F}$ & $\mathrm{N}$ & $C A$ \\
\hline 18 & Amity Creek & Duluth, Arnold & -- & $\mathrm{UM}$ & $0.3-1$ & $\mathrm{~N}$ & BUR/GAB & SD & F/A & $\mathrm{N}$ & $P R / A R$ \\
\hline 19 & Lester River & Duluth & -- & MM & $>1-2$ & E & GAB & $\mathrm{SD} / \mathrm{SH}$ & F/A & $\mathrm{N}$ & $P R$ \\
\hline m1 & Miller Creek & Duluth Heights & 44 & MM & $>4-8$ & B & GAB & BED/SH & $\mathbf{U}$ & $\mathbf{N}$ & CA \\
\hline m1a & Miller Creek & Duluth Heights & 36 & LM & $0.3-1$ & $\mathbf{E}$ & GAB & TP & $\mathbf{U}$ & $\mathbf{N}$ & PB/AR \\
\hline $\mathrm{m} 1 \mathrm{~b}$ & Miller Creek & Duluth Heights & -- & LM & $>8$ & $\mathrm{E}$ & GAB & BED & $\mathrm{U}$ & $\mathrm{N}$ & $\mathrm{BE}$ \\
\hline $\mathrm{m} 1 \mathrm{c}$ & Miller Creek & Duluth Heights & -- & LM & $>4-8$ & $\mathrm{E}$ & GAB & BED & $\mathrm{U}$ & $\mathrm{N}$ & $\mathrm{BE} / \mathrm{CA}$ \\
\hline m1d & Miller Creek & Duluth Heights & 7,37 & $\mathbf{L M}$ & $>8$ & B & GAB & BED & $\mathbf{U}$ & $\mathbf{N}$ & BE \\
\hline $\mathrm{mle}$ & Miller Creek & Duluth Heights & -- & LM & $>2-4$ & E & GAB & BED & $\mathrm{U}$ & $\mathrm{N}$ & $C A / S P$ \\
\hline $\mathbf{m} 2$ & Miller Creek & Duluth Heights & 35 & MM & $>1-2$ & B & GAB & SD/BED & $\mathbf{U}$ & $\mathbf{N}$ & PR/SP \\
\hline m3 & Miller Creek & Duluth Heights & 34 & MM & $0.3-1$ & $\mathbf{N}$ & GAB & SD/LA & $\mathbf{F} / \mathbf{U}$ & $\mathbf{N}$ & PR \\
\hline $\mathrm{m} 4$ & Miller Creek & Duluth Heights & 32,33 & UM & $0.3-1$ & $\mathbf{N}$ & BUR/GAB & SD & U/W & $\mathbf{N}$ & WE/AR \\
\hline $\mathrm{m} 5$ & Miller Creek & Duluth Heights & -- & UT & $0.3-1$ & $\mathrm{~N}$ & GAB & SD & $\mathrm{U}$ & $\mathrm{Y}$ & W/AR \\
\hline $\mathrm{m} 6$ & Miller Creek & Duluth Heights & -- & UT & $0.3-1$ & $\mathrm{~N}$ & GAB & SD & $\mathrm{U}$ & $\mathrm{Y}$ & $\mathrm{AR}$ \\
\hline $\mathbf{m} 7$ & Coffee Creek & Duluth Heights & 46 & $\mathbf{L T}$ & $>8$ & B & GAB & BED & $\mathbf{U}$ & $\mathbf{N}$ & $\mathrm{CO} / \mathrm{BE}$ \\
\hline $\mathrm{m} 8$ & Miller Creek & Duluth Heights & -- & $\mathrm{LT}$ & $>2-4$ & $\mathrm{C}$ & GAB & $\mathrm{BED} / \mathrm{SH}$ & $\mathrm{U}$ & $\mathrm{N}$ & $\mathrm{CO}$ \\
\hline m8a & Miller Creek & Duluth Heights & -- & LT & $>1-2$ & $\mathrm{C}$ & GAB & BED & $\mathrm{U}$ & $\mathrm{N}$ & $\mathrm{CO}$ \\
\hline $\mathrm{mp} 1$ & U.S. Steel Creek & West Duluth & -- & LM & $0.3-1$ & A & BUR & $\mathrm{TP}$ & $\mathrm{W} / \mathrm{U}$ & $\mathrm{N}$ & $\mathrm{AR} / \mathrm{WE}$ \\
\hline $\mathrm{mp} 2$ & U.S. Steel Creek & West Duluth & -- & MM & $>1-2$ & $\mathrm{C}$ & GAB & $\mathrm{TP}$ & $\mathrm{F} / \mathrm{U}$ & $\mathrm{N}$ & $\mathrm{PR} / S P$ \\
\hline $\mathrm{mp} 3$ & U.S. Steel Creek & West Duluth, Esko & -- & MM & $>2-4$ & $\mathrm{C}$ & GAB & $\mathrm{LA} / \mathrm{SH}$ & $\mathrm{F} / \mathrm{U}$ & $\mathrm{N}$ & $S P$ \\
\hline mp3a & U.S. Steel Creek & Esko & -- & $\mathrm{UM}$ & $>8$ & $\mathrm{C}$ & GAB/VOL & BED & $\mathrm{S} / \mathrm{F}$ & $Y$ & $\mathrm{BE}$ \\
\hline
\end{tabular}




\begin{tabular}{|c|c|c|c|c|c|c|c|c|}
\hline $\begin{array}{l}\text { Dominant } \\
\text { substrate }\end{array}$ & $\begin{array}{c}\text { Mode of } \\
\text { sediment transport }\end{array}$ & $\begin{array}{l}\text { Flood } \\
\text { attenuation }\end{array}$ & $\begin{array}{c}\text { Dominant } \\
\text { geomorphic } \\
\text { processes }\end{array}$ & $\begin{array}{c}\text { Observed/expected } \\
\text { causes for } \\
\text { disturbance }\end{array}$ & $\begin{array}{c}\text { Storm- } \\
\text { drain } \\
\text { outfalls }\end{array}$ & $\begin{array}{l}\text { Potential } \\
\text { geomorphic } \\
\text { changes }\end{array}$ & $\begin{array}{c}\text { Sensitivity } \\
\text { to } \\
\text { disturbance }\end{array}$ & $\begin{array}{c}\text { Geomorphic } \\
\text { segment } \\
\text { category }\end{array}$ \\
\hline $\mathrm{BO} / \mathrm{BE}$ & T, SIc & NO & EX, W & UP, HI & $\mathbf{N}$ & $\mathbf{Y}$ & LO & $\mathbf{L 1}$ \\
\hline BE & T, SIp & No & $\mathbf{E X}, \mathbf{W}$ & UP, HI & $\mathbf{N}$ & $\mathbf{Y}$ & LO & L2 \\
\hline$B O$ & $\mathrm{~T}, \mathrm{SIc}, \mathrm{SOw}, \mathrm{SOb}$ & NO & EX, W & UP, HI, RO, CL & $\mathrm{N}$ & $\mathrm{Y}$ & $H I / M O D$ & M1 \\
\hline BO & SOi, SOw, SOc & NO & I, EBL, EBA & CL, UR, RO & $\mathrm{N}$ & Y & $\mathrm{HI}$ & BT \\
\hline$B O$ & SOi, SOw, SOc & NO & I, EBL, EBA & CL, UR, RO & $\mathrm{N}$ & Y & MOD & BT \\
\hline$B O$ & SOi, SOw, SOc & NO & I, EBL, EBA & UR, RO & $\mathrm{Y}$ & $\mathrm{Y}$ & MOD & BT \\
\hline BO & SOi, SOw, SOc & NO & I, EBL, EBA & UR, RO & $\mathrm{Y}$ & $\mathrm{Y}$ & MOD & BT \\
\hline $\mathrm{CO}$ & SOw, SOi & NO & I, EBA, LM & CL, UR, RO & $\mathrm{N}$ & $\mathrm{Y}$ & MOD/HI & LT \\
\hline $\mathrm{CO}$ & SOw, SOi & NO & I, EBA, LM & CL, UR, RO & $\mathrm{N}$ & Y & MOD/HI & LT \\
\hline$B O$ & SOw & NO & I, EBA & $\mathrm{CL}$ & $\mathrm{N}$ & $\mathrm{Y}$ & MOD & $\mathrm{BT}$ \\
\hline$B O$ & SOw & NO & EX, W & $\mathrm{CL}$ & $\mathrm{N}$ & $\mathrm{Y}$ & MOD & BT \\
\hline$B O$ & SOw, SOi, SOb & $\mathrm{NO}$ & I, EBA, LM, W & $\mathrm{CL}$ & $\mathrm{N}$ & $\mathrm{Y}$ & $\mathrm{HI}$ & LT \\
\hline$B O / B E$ & SOw, SOi, SOb & NO & I, EBA, LM, W & $\mathrm{CL}$ & $\mathrm{N}$ & $\mathrm{Y}$ & MOD & $\mathrm{BT}$ \\
\hline$G V$ & T, SOw, SOb & $F P$ & I, EBL, EBA & UP, GV & $\mathrm{N}$ & $\mathrm{Y}$ & $H I$ & M.3 \\
\hline $\mathrm{CO} / \mathrm{BO}$ & $\mathrm{T}$ & NO & EX, EBA, W & UP & $\mathrm{N}$ & $\mathrm{Y}$ & MOD & M1 \\
\hline$B O$ & $T$ & NO & EX, EBA, W & $\mathrm{UP}, \mathrm{CL}$ & $\mathrm{N}$ & $\mathrm{Y}$ & MOD & M2 \\
\hline$G V$ & SOw & NO & EBA, W, LM & $\mathrm{CL}, \mathrm{CA}$ & $\mathrm{N}$ & Y & $M O D / L O$ & W. 3 \\
\hline$G V$ & SOw, SOb & NO & EBA, EBL, W, LM & UP, CA & $\mathrm{N}$ & Y & $H I / M O D$ & M1 \\
\hline BO/BE & $\mathrm{T}, \mathrm{SOb}, \mathrm{SO}$ & No & EBL, W, EX, BF & UP, UR, HI, RO, CL & $\mathbf{Y}$ & $\mathbf{Y}$ & MOD & B \\
\hline BO & $\mathbf{T}$ & NO & ST & UP & $\mathbf{Y}$ & $\mathbf{Y}$ & LO & $\mathbf{A}$ \\
\hline $\mathrm{BE}$ & $\mathrm{T}, \mathrm{SOb}$ & NO & EBL, EX & UR, UP, HI & $\mathrm{Y}$ & $\mathrm{Y}$ & LO & B \\
\hline $\mathrm{BE} / \mathrm{BO}$ & $\mathrm{T}, \mathrm{SOb}$ & NO & EBL, EX & UR, UP, HI & $\mathrm{Y}$ & $\mathrm{Y}$ & LO & B \\
\hline BE & T, SOb, SOi & NO & EBL, EX, I & UR, UP, HI & $\mathbf{Y}$ & $\mathbf{Y}$ & LO & B \\
\hline $\mathrm{BO}$ & $\mathrm{T}, \mathrm{SOb}$ & NO & EBL, EX & UP, UR, HI & $\mathrm{Y}$ & Y & LO & L2 \\
\hline BO & SOw, T, SIc & NO & EBA, W, LM & UP, UR, RO, CL & $\mathbf{Y}$ & $\mathbf{Y}$ & MOD & M1 \\
\hline BO/GV & $\begin{array}{l}\text { SOw, T, SOi, SIc, } \\
\text { SIb }\end{array}$ & NO & $\begin{array}{l}\text { EBA, W, LM, I, BF, } \\
\quad \text { EBL }\end{array}$ & UP, UR, RO, CA, CL & $\mathbf{Y}$ & $\mathbf{Y}$ & HI & M.3 \\
\hline SA/FINES & SOw, SOi, SIa & WE & EBA, W, A, I & UR, RO, CA & $\mathbf{Y}$ & $\mathbf{Y}$ & MOD & W.3 \\
\hline SA/FINES & SOw, SOi, SIa & WE & EBA, W, A, I & $\mathrm{UR}, \mathrm{RO}, \mathrm{CA}, \mathrm{CL}$ & $\mathrm{N}$ & $\mathrm{Y}$ & MOD & W.3 \\
\hline SA/FINES & SOw, SOi, SIa & WE & EBA, W, A, I & $\mathrm{UR}, \mathrm{RO}, \mathrm{CA}, \mathrm{CL}$ & $\mathrm{N}$ & $\mathrm{Y}$ & MOD & W.3 \\
\hline BE & T, SOb & No & EX, EBL, W & UP, UR, HI & $\mathbf{Y}$ & $\mathbf{Y}$ & LO & BT \\
\hline BO & SOw & NO & $\begin{array}{l}\mathrm{EX}, \mathrm{EBA}, \mathrm{W}, \mathrm{LM}, \\
\quad E B L\end{array}$ & GV, UR, RO & $\mathrm{N}$ & $\mathrm{Y}$ & $\mathrm{HI}$ & $\mathrm{LT}$ \\
\hline $\mathrm{CO}$ & SOw, T, SIp & NO & W, EBA, LM, $B F$ & $\mathrm{CL}, \mathrm{UR}, \mathrm{CA}$ & $\mathrm{N}$ & $\mathrm{Y}$ & MOD & LT \\
\hline $\mathrm{SA} / F I N E S$ & T, SIa, SOw & WE/FP & $\mathrm{A}, E B A, W$ & UP, RO, UR, CA & $\mathrm{Y}$ & $\mathrm{Y}$ & MOD & A \\
\hline $\mathrm{BO} / G V$ & $\mathrm{~T}, S O w, S O b$ & NO & $\mathrm{W}, E B A, E B L, L M$ & UP, UR, RO & $\mathrm{Y}$ & Y & MOD & M1 \\
\hline$B O$ & T, SOw, SOb & NO & EBL, EBA, LM, BF & UP, RO, GV, UR & $\mathrm{N}$ & $\mathrm{Y}$ & $\mathrm{HI}$ & M2 \\
\hline $\mathrm{BE}$ & SOw & $\mathrm{NO}$ & EX & UP, UR, RO & $\mathrm{N}$ & $\mathrm{Y}$ & LO & $\mathrm{B}$ \\
\hline
\end{tabular}


Table 5. Geomorphic characteristics of stream segments for Duluth-area streams, Minn., 2003-2004—Continued

[See table 6 for explanation of abbreviations. Stream segments are shown on figure 13. Data in italics are extrapolated from reach assessments of sites with the same segment category. Data in bold are rapid assessment or intensive survey sites. USGS, U.S. Geological Survey; ID, identification number; --, no detailed measurements taken; \%, percent; >, greater than; <, less than]

\begin{tabular}{|c|c|c|c|c|c|c|c|c|c|c|c|}
\hline $\begin{array}{l}\text { Segment } \\
\text { ID }\end{array}$ & Stream name & $\begin{array}{l}\text { Topographic } \\
\text { map }\end{array}$ & $\begin{array}{c}\text { USGS } \\
\text { reach } \\
\text { ID }\end{array}$ & $\begin{array}{c}\text { Drainage- } \\
\text { network } \\
\text { position }\end{array}$ & $\begin{array}{c}\text { Slope } \\
\%\end{array}$ & $\begin{array}{c}\text { Valley } \\
\text { type }\end{array}$ & $\begin{array}{c}\text { Bedrock } \\
\text { type }\end{array}$ & $\begin{array}{c}\text { Glacial } \\
\text { landform }\end{array}$ & $\begin{array}{l}\text { Dominant } \\
\text { land cover }\end{array}$ & $\begin{array}{c}\text { Inter- } \\
\text { mittent }\end{array}$ & $\begin{array}{c}\text { Channel } \\
\text { type }\end{array}$ \\
\hline mp4 & U.S. Steel Creek & West Duluth & -- & MT & $>4-8$ & $\mathrm{C}$ & BUR/GAB & BED & S & $\mathrm{N}$ & $\mathrm{AR}$ \\
\hline mp5 & U.S. Steel Creek & West Duluth & 45 & MM & $>1-2$ & B & GAB & BED & $\mathbf{U} / \mathbf{F}$ & $\mathbf{N}$ & PB/AR \\
\hline mp6 & U.S. Steel Creek & West Duluth & -- & UT & $>2-4$ & $\mathrm{C}$ & GAB & BED & F & $\mathrm{N}$ & $\mathrm{CA} / \mathrm{SP}$ \\
\hline $\mathrm{mp} 7$ & U.S. Steel Creek & West Duluth & -- & UT & $>4-8$ & $\mathrm{C}$ & GAB & BED & F & $\mathrm{N}$ & $\mathrm{CO} / B E$ \\
\hline mp8 & U.S. Steel Creek & West Duluth & -- & UM & $>2-4$ & $\mathrm{C}$ & GAB & BED & F & $\mathrm{N}$ & $P B$ \\
\hline mp9 & U.S. Steel Creek & West Duluth & -- & UT & $>8$ & $\mathrm{C}$ & VOL & BED & F & $\mathrm{N}$ & $\mathrm{BE}$ \\
\hline ms1 & Mission Creek & Esko & 38 & LM & $0.3-1$ & $\mathbf{A}$ & SED & $\mathbf{L A}$ & G & $\mathbf{N}$ & PB/AR \\
\hline $\mathrm{ms} 10$ & Mission Creek & Esko & -- & MT & $>1-2$ & $\mathrm{E}$ & SED & BED & F & Y & $P B$ \\
\hline $\mathrm{ms} 11$ & Mission Creek & Esko & -- & MT & $>2-4$ & $\mathrm{E}$ & SED & $\mathrm{LA} / \mathrm{SH}$ & $F / R$ & $\mathrm{Y}$ & $\mathrm{CO}$ \\
\hline ms11a & Mission Creek & Esko & -- & UT & $>2-4$ & $\mathrm{C}$ & VOL & $\mathrm{BED} / \mathrm{SH}$ & $\mathrm{R}$ & Y & $P B / A R$ \\
\hline $\mathrm{ms} 12$ & Mission Creek & Esko & -- & MT & $>2-4$ & $\mathrm{C}$ & SED & $\mathrm{SH}$ & $\mathrm{R}$ & $\mathrm{Y}$ & $\mathrm{CO}$ \\
\hline $\mathrm{ms} 13$ & Mission Creek & Esko & -- & MT & $>2-4$ & $\mathrm{C}$ & SED & $\mathrm{SH}$ & $\mathrm{R}$ & $\mathrm{Y}$ & $\mathrm{CO}$ \\
\hline $\mathrm{ms} 14$ & Mission Creek & Esko & -- & MT & $>2-4$ & $\mathrm{C}$ & SED & $\mathrm{SH}$ & F & $\mathrm{Y}$ & $\mathrm{CO}$ \\
\hline $\mathrm{ms} 15$ & Mission Creek & Esko & -- & MT & $>2-4$ & $\mathrm{C}$ & SED & $\mathrm{SH}$ & $\mathrm{R}$ & $\mathrm{Y}$ & $\mathrm{CO}$ \\
\hline $\mathrm{ms} 16$ & Mission Creek & Esko & -- & UT & $>2-4$ & $\mathrm{C}$ & SED & $\mathrm{SH}$ & $S / R$ & $\mathrm{Y}$ & $\mathrm{CO}$ \\
\hline $\mathrm{ms} 17$ & Mission Creek & Esko & -- & MT & $>2-4$ & $\mathrm{E}$ & SED & $\mathrm{BED} / \mathrm{SH}$ & $\mathrm{F} / \mathrm{R}$ & $\mathrm{Y}$ & $P B$ \\
\hline $\mathrm{ms} 18$ & Mission Creek & Esko & -- & MT & $>2-4$ & $\mathrm{C}$ & SED & SH & $\mathrm{F} / \mathrm{R}$ & $\mathrm{Y}$ & $\mathrm{CO}$ \\
\hline $\mathrm{ms} 19$ & Mission Creek & Esko & -- & MT & $>2-4$ & $\mathrm{E}$ & SED & $\mathrm{BED} / \mathrm{SH}$ & F & Y & $P B$ \\
\hline $\mathrm{ms} 2$ & Mission Creek & Esko & -- & MM & $>1-2$ & $\mathrm{E}$ & SED & BED/LA & F & $\mathrm{N}$ & $P B$ \\
\hline $\mathrm{ms} 20$ & Mission Creek & Esko & -- & MT & $>2-4$ & $\mathrm{C}$ & SED & $\mathrm{BED} / \mathrm{SH}$ & F & $\mathrm{Y}$ & $\mathrm{CO}$ \\
\hline $\mathrm{ms} 21$ & Mission Creek & Esko & -- & MT & $>2-4$ & $\mathrm{C}$ & SED & $\mathrm{BED} / \mathrm{SH}$ & $\mathrm{F}$ & Y & $\mathrm{CO}$ \\
\hline $\mathrm{ms} 22$ & Mission Creek & Esko & -- & MT & $>4-8$ & $\mathrm{C}$ & SED & BED & F & $\mathrm{Y}$ & $\mathrm{CO}$ \\
\hline $\mathrm{ms} 23$ & Mission Creek & Esko & -- & MT & $>1-2$ & $\mathrm{C}$ & SED & $\mathrm{BED} / \mathrm{SH}$ & F & Y & $\mathrm{CO}$ \\
\hline $\mathrm{ms} 24$ & Mission Creek & Esko & -- & MT & $>2-4$ & $\mathrm{E}$ & SED & $\mathrm{BED} / \mathrm{SH}$ & $\mathrm{F}$ & $\mathrm{Y}$ & $P B$ \\
\hline $\mathrm{ms} 25$ & Mission Creek & Esko & -- & LT & $>2-4$ & $\mathrm{C}$ & SED & $\mathrm{BED} / \mathrm{SH}$ & $F / R$ & $\mathrm{Y}$ & $\mathrm{CO}$ \\
\hline $\mathrm{ms} 26$ & Mission Creek & Esko & -- & LT & $>2-4$ & $\mathrm{C}$ & SED & BED & F & $\mathrm{Y}$ & $\mathrm{CO}$ \\
\hline $\mathrm{ms} 27$ & Mission Creek & Esko & -- & $\mathrm{LT}$ & $>2-4$ & $\mathrm{C}$ & SED & BED & F & $\mathrm{Y}$ & $\mathrm{CO}$ \\
\hline $\mathrm{ms} 2 \mathrm{a}$ & Mission Creek & Esko & -- & LM & $>1-2$ & $\mathrm{E}$ & SED & $\mathrm{BED} / \mathrm{LA}$ & $\mathrm{F}$ & $\mathrm{N}$ & $\mathrm{PR} / \mathrm{PB}$ \\
\hline $\mathrm{ms} 2 \mathrm{~b}$ & Mission Creek & Esko & 39 & LM & $>2-4$ & $\mathbf{A}$ & SED & BED & $\mathbf{F}$ & $\mathbf{N}$ & PB/PR \\
\hline $\mathrm{ms} 3$ & Mission Creek & Esko & -- & MM & $>2-4$ & $\mathrm{E}$ & SED & BED & $\mathrm{F}$ & $\mathrm{N}$ & $P B / P R$ \\
\hline ms4 & Mission Creek & Esko & 1 & MM & $>1-2$ & $\mathbf{E}$ & SED & SH//TP & F/S & $\mathbf{N}$ & PR \\
\hline ms5 & Mission Creek & Esko & 40 & UM & $0.3-1$ & $\mathbf{N}$ & BUR/SED & $\mathbf{T P}$ & $\mathbf{R} / \mathrm{S} / \mathbf{F}$ & $\mathbf{N}$ & WE \\
\hline ms6 & Mission Creek & Esko & -- & UT & $0.3-1$ & $\mathrm{~N}$ & SED & TI & $\mathrm{R} / \mathrm{S} / \mathrm{F}$ & $\mathrm{Y}$ & WE \\
\hline ms7 & Mission Creek & Esko & -- & UT & $0.3-1$ & $\mathrm{~N}$ & SED & $\mathrm{TI}$ & $\mathrm{R} / \mathrm{S} / \mathrm{F}$ & $\mathrm{Y}$ & WE \\
\hline $\mathrm{ms} 8$ & Mission Creek & Esko & -- & MT & $>1-2$ & $\mathrm{C}$ & SED & SH & $\mathrm{F} / \mathrm{S}$ & $\mathrm{Y}$ & $P B$ \\
\hline $\mathrm{ms} 9$ & Mission Creek & Esko & -- & MT & $>1-2$ & $\mathrm{E}$ & SED & BED & $\mathrm{F}$ & $\mathrm{Y}$ & $P B$ \\
\hline o1 & Merritt Creek & Duluth Heights & -- & LM & $>1-2$ & $\mathrm{~N}$ & GAB & $\mathrm{TP}$ & $\mathrm{U}$ & $\mathrm{N}$ & $\mathrm{PB} / \mathrm{AR}$ \\
\hline $\mathrm{o} 2$ & Merritt Creek & Duluth Heights & -- & LM & $>4-8$ & $\mathrm{C}$ & GAB & BED & $\mathrm{U}$ & $\mathrm{N}$ & $\mathrm{CA}$ \\
\hline 03 & Merritt Creek & Duluth Heights & -- & MM & $>8$ & $\mathrm{E}$ & GAB & BED & S & $\mathrm{N}$ & $\mathrm{BE}$ \\
\hline
\end{tabular}




\begin{tabular}{|c|c|c|c|c|c|c|c|c|}
\hline $\begin{array}{l}\text { Dominant } \\
\text { substrate }\end{array}$ & $\begin{array}{c}\text { Mode of } \\
\text { sediment transport }\end{array}$ & $\begin{array}{l}\text { Flood } \\
\text { attenuation }\end{array}$ & $\begin{array}{l}\text { Dominant } \\
\text { geomorphic } \\
\text { processes }\end{array}$ & $\begin{array}{l}\text { Observed/expected } \\
\text { causes for } \\
\text { disturbance }\end{array}$ & $\begin{array}{c}\text { Storm- } \\
\text { drain } \\
\text { outfalls }\end{array}$ & $\begin{array}{l}\text { Potential } \\
\text { geomorphic } \\
\text { changes }\end{array}$ & $\begin{array}{c}\text { Sensitivity } \\
\text { to } \\
\text { disturbance }\end{array}$ & $\begin{array}{c}\text { Geomorphic } \\
\text { segment } \\
\text { category }\end{array}$ \\
\hline $\mathrm{CA} / \mathrm{BE}$ & SOw, SOi & NO & EBA, I, W, BF & $\mathrm{GV}, \mathrm{CA}, \mathrm{UR}$ & $\mathrm{N}$ & $\mathrm{Y}$ & MOD & BT \\
\hline BE/BO & T, SOw & No & EX, EBA & UP, RO, GV, CA & $\mathbf{N}$ & $\mathbf{Y}$ & MOD & M1 \\
\hline$B O$ & SOi, SOw & NO & EBA, I, W & UR, RO & $\mathrm{N}$ & Y & MOD & $\mathrm{T} 2$ \\
\hline $\mathrm{BO} / B E$ & SOi, SOw & NO & EX, EBA, I, W & RO & $\mathrm{N}$ & $\mathrm{Y}$ & MOD & BT \\
\hline$B O$ & T, SOw, SOb & NO & EBA, W, I, EBL & $\mathrm{RO}, \mathrm{CL}$ & $\mathrm{N}$ & $\mathrm{Y}$ & MOD & $\mathrm{U} 2$ \\
\hline $\mathrm{BE}$ & SOw, SOi & NO & $\mathrm{EX}, E B A, W$ & $\mathrm{CL}$ & $\mathrm{N}$ & $\mathrm{Y}$ & LO & BT \\
\hline $\mathrm{CO}$ & $\mathbf{T}$ & NO & W, EBA & $\mathbf{U P}$ & $\mathbf{Y}$ & $\mathbf{Y}$ & LO & $\mathbf{A}$ \\
\hline $\mathrm{CO} / \mathrm{BO}$ & SOi, SOb, SOw & NO & I, W, EBA, EBL & $\mathrm{CL}$ & $\mathrm{N}$ & $\mathrm{Y}$ & HI & $\mathrm{T} 1$ \\
\hline $\mathrm{CO}$ & SOi, SOb, SOw & NO & I, W, EBA, EBL & $\mathrm{CL}, \mathrm{RO}$ & $\mathrm{N}$ & $\mathrm{Y}$ & HI & $\mathrm{T} 2$ \\
\hline $\mathrm{BO} / \mathrm{CO}$ & SOi, SOb, SOw & NO & I, W, EBA, EBL & $\mathrm{RO}$ & $\mathrm{N}$ & Y & HI & $\mathrm{T} 2$ \\
\hline $\mathrm{CO}$ & SOi, SOb, SOw & NO & I, W, EBA, EBL & RO & $\mathrm{N}$ & $\mathrm{Y}$ & HI & $\mathrm{T} 2$ \\
\hline $\mathrm{CO}$ & SOi, SOb, SOw & NO & I, W, EBA, EBL & $\mathrm{RO}$ & $\mathrm{N}$ & $\mathrm{Y}$ & $\mathrm{HI}$ & $\mathrm{T} 2$ \\
\hline $\mathrm{CO}$ & SOi, SOb, SOw & NO & I, W, EBA, EBL & CL, RO & $\mathrm{N}$ & Y & MOD & $\mathrm{T} 2$ \\
\hline $\mathrm{CO}$ & SOi, SOb, SOw & NO & I, EBL, EBA & $\mathrm{CL}, \mathrm{RO}$ & $\mathrm{N}$ & $\mathrm{Y}$ & $\mathrm{HI}$ & $\mathrm{T} 2$ \\
\hline $\mathrm{CO}$ & SOi, SOb, SOw & NO & I, EBL, EBA & CL, RO & $\mathrm{N}$ & $\mathrm{Y}$ & $\mathrm{HI}$ & $\mathrm{T} 2$ \\
\hline $\mathrm{CO}$ & SOi, SOb, SOw & NO & I, EBL, EBA & $\mathrm{CL}, \mathrm{RO}$ & $\mathrm{N}$ & Y & $\mathrm{HI}$ & $\mathrm{T} 2$ \\
\hline $\mathrm{CO}$ & SOi, SOb, SOw & NO & I, EBL, EBA & $\mathrm{CL}, \mathrm{RO}$ & $\mathrm{N}$ & Y & $\mathrm{HI}$ & $\mathrm{T} 2$ \\
\hline $\mathrm{CO}$ & SOi, SOb, SOw & NO & I, EBL, EBA & UP, CL, UR & $\mathrm{N}$ & Y & MOD & $\mathrm{T} 2$ \\
\hline$G V$ & $\mathrm{~T}$ & NO & $\begin{array}{l}\text { EBL, EBA, W, LM, } \\
\text { BF }\end{array}$ & UP & $\mathrm{N}$ & Y & MOD & M1 \\
\hline $\mathrm{CO}$ & SOi, SOb, SOw & NO & I, EBL, EBA, LM & UP, CL, RO, UR & $\mathrm{N}$ & $\mathrm{Y}$ & $\mathrm{HI}$ & $\mathrm{T} 2$ \\
\hline $\mathrm{CO}$ & SOi, SOb, SOw & NO & I, EBL, EBA, LM & $\mathrm{CL}, \mathrm{RO}, \mathrm{UR}$ & $\mathrm{N}$ & $\mathrm{Y}$ & $\mathrm{HI}$ & $\mathrm{T} 2$ \\
\hline$B O$ & $\mathrm{~T}, \mathrm{SOb}, \mathrm{SOw}$ & NO & EBA, EBL & $\mathrm{NO}$ & $\mathrm{N}$ & $\mathrm{N}$ & MOD & $\mathrm{BT}$ \\
\hline $\mathrm{CO} / \mathrm{BO}$ & SOi, SOb, SOw & NO & EBA, EBL & NO & $\mathrm{N}$ & $\mathrm{N}$ & MOD & $\mathrm{T} 1$ \\
\hline $\mathrm{CO}$ & $\mathrm{SOi}, \mathrm{SOb}, \mathrm{SOw}$ & NO & I, EBA, EBL & $\mathrm{NO}$ & $\mathrm{N}$ & $\mathrm{N}$ & MOD & $\mathrm{T} 2$ \\
\hline $\mathrm{CO}$ & SOi, SOb, SOw & NO & I, EBA, EBL & $\mathrm{NO}$ & $\mathrm{N}$ & $\mathrm{N}$ & MOD/HI & LT \\
\hline $\mathrm{CO}$ & SOi, SOb, SOw & NO & I, EBA, EBL & NO & $\mathrm{N}$ & $\mathrm{N}$ & MOD/HI & $\mathrm{LT}$ \\
\hline $\mathrm{CO}$ & SOi, SOb, SOw & NO & I, EBA, EBL & $\mathrm{NO}$ & $\mathrm{N}$ & $\mathrm{N}$ & $\mathrm{MOD} / \mathrm{HI}$ & LT \\
\hline GV & T, SIc, SOw & NO & $\mathrm{W}, \mathrm{EBA}, \mathrm{BF}$ & UP & $\mathrm{N}$ & $\mathrm{Y}$ & MOD & $\mathrm{L} 1$ \\
\hline CO/GV & T, SIc & FP & W, EBA, BF & UP & $\mathbf{N}$ & $\mathbf{Y}$ & MOD & $\mathbf{L 2}$ \\
\hline$C O / G V$ & T, $S O w, S O b$ & NO & $E B L, E B A, E X$ & UP & $\mathrm{N}$ & $\mathrm{Y}$ & $\mathrm{HI}$ & M2 \\
\hline GV & SOb, SOw, T & FP,NO & EBL, EBA, W, LM, I & UP, RO & $\mathbf{N}$ & $\mathbf{Y}$ & HI & M1 \\
\hline GV & SOi & WE & I, W, EBA, A & CL, RO & $\mathbf{N}$ & $\mathbf{Y}$ & MOD & W.3 \\
\hline GV/FINES & $\mathrm{SO}$ & WE & I, W, EBA, A & $\mathrm{CL}, \mathrm{RO}$ & $\mathrm{N}$ & $\mathrm{Y}$ & MOD & W.3 \\
\hline GV/FINES & SOi & WE & I, W, EBA, A & $\mathrm{CL}, \mathrm{RO}$ & $\mathrm{N}$ & $\mathrm{Y}$ & MOD & W.3 \\
\hline $\mathrm{CO} / \mathrm{BO}$ & SOi, SOb, SOw & NO & I, W, EBL, EBA & CL, RO & $\mathrm{N}$ & $\mathrm{Y}$ & $\mathrm{HI}$ & $\mathrm{T} 1$ \\
\hline $\mathrm{CO} / \mathrm{BO}$ & $\mathrm{SOi}, \mathrm{SOb}, \mathrm{SOw}$ & NO & I, W, EBL, EBA & $\mathrm{CL}, \mathrm{RO}$ & $\mathrm{N}$ & $\mathrm{Y}$ & $\mathrm{HI}$ & $\mathrm{T} 1$ \\
\hline$B O$ & $\mathrm{~T}$ & NO & W, $E B A, E B L$ & UP, RO, UR & $\mathrm{Y}$ & $\mathrm{Y}$ & LO & L1 \\
\hline BO & T, SOw, SOb & NO & EX & UP, RO, UR, CL & $\mathrm{Y}$ & $\mathrm{Y}$ & LO & B \\
\hline $\mathrm{BE}$ & T, SOw, SOb & NO & EX & UP, RO, CL, UR & $\mathrm{Y}$ & $\mathrm{Y}$ & LO & $\mathrm{B}$ \\
\hline
\end{tabular}


Table 5. Geomorphic characteristics of stream segments for Duluth-area streams, Minn., 2003-2004-Continued

[See table 6 for explanation of abbreviations. Stream segments are shown on figure 13. Data in italics are extrapolated from reach assessments of sites with the same segment category. Data in bold are rapid assessment or intensive survey sites. USGS, U.S. Geological Survey; ID, identification number; --, no detailed measurements taken; \%, percent; $>$, greater than; $<$, less than]

\begin{tabular}{|c|c|c|c|c|c|c|c|c|c|c|c|}
\hline $\begin{array}{l}\text { Segment } \\
\text { ID }\end{array}$ & Stream name & $\begin{array}{l}\text { Topographic } \\
\text { map }\end{array}$ & $\begin{array}{l}\text { USGS } \\
\text { reach } \\
\text { ID }\end{array}$ & $\begin{array}{c}\text { Drainage- } \\
\text { network } \\
\text { position }\end{array}$ & $\begin{array}{c}\text { Slope } \\
\%\end{array}$ & $\begin{array}{c}\text { Valley } \\
\text { type }\end{array}$ & $\begin{array}{c}\text { Bedrock } \\
\text { type }\end{array}$ & $\begin{array}{l}\text { Glacial } \\
\text { landform }\end{array}$ & $\begin{array}{l}\text { Dominant } \\
\text { land cover }\end{array}$ & $\begin{array}{l}\text { Inter- } \\
\text { mittent }\end{array}$ & $\begin{array}{c}\text { Channel } \\
\text { type }\end{array}$ \\
\hline 04 & Merritt Creek & Duluth Heights & 48 & MM & $>8$ & $\mathbf{E}$ & GAB & BED & $\mathbf{U}$ & $\mathbf{N}$ & BE \\
\hline o5 & Merritt Creek & Duluth Heights & -- & UM & $>2-4$ & E & GAB & SH/BED & $\mathrm{U}$ & $\mathrm{N}$ & PB \\
\hline 06 & Merritt Creek & Duluth Heights & 47 & MT & $>8$ & $\mathbf{E}$ & GAB & BED & $\mathbf{F}$ & $\mathbf{N}$ & BE \\
\hline 07 & Merritt Creek & Duluth Heights & -- & UT & $>2-4$ & $\mathrm{C}$ & GAB & SH/BED & $\mathrm{F} / \mathrm{U}$ & $Y$ & $\mathrm{~PB} / \mathrm{SP}$ \\
\hline s1 & Stewart Creek & West Duluth & -- & LM & $>1-2$ & $\mathrm{~N}$ & GAB & $\mathrm{TP}$ & $\mathrm{W} / \mathrm{U}$ & $\mathrm{N}$ & $A R / P B$ \\
\hline s2 & Stewart Creek & West Duluth & 3 & LM & $>8$ & B & GAB & BED/SH & $F / U$ & $\mathbf{N}$ & $\mathrm{BE} / \mathrm{CA}$ \\
\hline s3 & Stewart Creek & West Duluth & -- & MM & $>2-4$ & $\mathrm{C}$ & GAB & BED & F & $\mathrm{N}$ & $P B$ \\
\hline s4 & Stewart Creek & West Duluth & -- & MT & $>2-4$ & $\mathrm{C}$ & GAB & $B E D / T P$ & $\mathrm{~F}$ & $\mathrm{~N}$ & $\mathrm{CO}$ \\
\hline s5 & Stewart Creek & West Duluth, Esko & -- & UM & $>4-8$ & $\mathrm{E}$ & GAB & $B E D / T P$ & $\mathrm{~F}$ & $\mathrm{~N}$ & $C A / A R$ \\
\hline $\mathrm{s} 5 \mathrm{a}$ & Stewart Creek & West Duluth, Esko & -- & UT & $>2-4$ & $\mathrm{~N}$ & VOL & $B E D / T P$ & $\mathrm{U} / \mathrm{F}$ & $\mathrm{Y}$ & $P B / S P$ \\
\hline s6 & Stewart Creek & West Duluth & 2 & LT & $>4-8$ & B & GAB & BED/SH & $\mathbf{F}$ & $\mathbf{N}$ & $\mathrm{CO}$ \\
\hline $\operatorname{sg} 1$ & Sargent Creek & West Duluth & -- & LM & $0.3-1$ & A & SED & LA & $\mathrm{F} / \mathrm{U}$ & $\mathrm{N}$ & $P R$ \\
\hline $\operatorname{sg} 2$ & Sargeant Creek & West Duluth, Esko & -- & MM & $>1-2$ & $\mathrm{C}$ & SED/VOL & $\mathrm{LA} / \mathrm{SH}$ & $\mathrm{F}$ & $\mathrm{N}$ & $\mathrm{PR} / \mathrm{SP}$ \\
\hline $\operatorname{sg} 2 \mathrm{a}$ & Sargeant Creek & West Duluth, Esko & -- & MM & $>1-2$ & E & SED & LA & $\mathrm{F} / \mathrm{U}$ & $\mathrm{N}$ & PR \\
\hline $\operatorname{sg} 2 b$ & Sargent Creek & West Duluth, Esko & -- & LM & $0.3-1$ & $\mathrm{C}$ & SED & LA & $\mathrm{F} / \mathrm{U}$ & $\mathrm{N}$ & $\mathrm{PR}$ \\
\hline $\operatorname{sg} 3$ & Sargeant Creek & Esko & -- & UM & $>2-4$ & $\mathrm{C}$ & VOL & LA & $\mathrm{F}$ & $\mathrm{N}$ & $P B / S P$ \\
\hline $\operatorname{sg} 4$ & Sargeant Creek & Esko & -- & MT & $>2-4$ & $\mathrm{C}$ & VOL & LA & $\mathrm{F} / \mathrm{U}$ & $\mathrm{Y}$ & $C A / S P$ \\
\hline $\operatorname{sg} 5$ & Sargeant Creek & Esko & -- & MT & $>4-8$ & $\mathrm{C}$ & VOL & LA & $\mathrm{U} / \mathrm{F}$ & $\mathrm{Y}$ & $B E / C O$ \\
\hline $\operatorname{sg} 6$ & Sargeant Creek & Esko & -- & MT & $>8$ & $\mathrm{C}$ & VOL & $\mathrm{BED} / \mathrm{SH}$ & $\mathrm{F}$ & $\mathrm{Y}$ & $\mathrm{BE}$ \\
\hline svla & $\begin{array}{l}\text { Lenroot St Creek, } \\
\text { Gogebic St Creek, } \\
\text { 84th Ave W } \\
\text { Creek, 85th Ave } \\
\text { W Creek }\end{array}$ & West Duluth & -- & LM & $>2-4$ & $\mathrm{C}$ & BUR & $\mathrm{TP}$ & $\mathrm{F} / \mathrm{U}$ & $\mathrm{N}$ & $A R / P B$ \\
\hline sv1b & $\begin{array}{l}\text { Lenroot St Creek, } \\
\text { Gogebic St Creek, } \\
\text { 84th Ave W } \\
\text { Creek, 85th Ave } \\
\text { W Creek }\end{array}$ & West Duluth & -- & MM & $>8$ & $\mathrm{C}$ & GAB & BED & $\mathrm{F} / \mathrm{U}$ & $\mathrm{N}$ & $B E$ \\
\hline sv2 & $\begin{array}{l}\text { Lenroot St Creek, } \\
\text { Gogebic St Creek, } \\
\text { 84th Ave W } \\
\text { Creek, 85th Ave } \\
\text { W Creek }\end{array}$ & West Duluth & -- & UM & $>2-4$ & $\mathrm{C}$ & GAB & SH/BED & $\mathrm{F}$ & $\mathrm{Y}$ & $P B$ \\
\hline sv3a & $\begin{array}{l}\text { Lenroot St Creek, } \\
\text { Gogebic St Creek, } \\
\text { 84th Ave W } \\
\text { Creek, 85th Ave } \\
\text { W Creek }\end{array}$ & West Duluth & -- & LM & $>2-4$ & $\mathrm{C}$ & BUR & $\mathrm{TP}$ & $\mathrm{F} / \mathrm{U}$ & $\mathrm{N}$ & $A R / P B$ \\
\hline $\mathrm{sv} 3 \mathrm{~b}$ & $\begin{array}{l}\text { Lenroot St Creek, } \\
\text { Gogebic St Creek, } \\
\text { 84th Ave W } \\
\text { Creek, 85th Ave } \\
\text { W Creek }\end{array}$ & West Duluth & -- & MM & $>8$ & C & GAB & BED & F & $\mathrm{N}$ & $\mathrm{BE}$ \\
\hline
\end{tabular}




\begin{tabular}{|c|c|c|c|c|c|c|c|c|}
\hline $\begin{array}{l}\text { Dominant } \\
\text { substrate }\end{array}$ & $\begin{array}{c}\text { Mode of } \\
\text { sediment transport }\end{array}$ & $\begin{array}{c}\text { Flood } \\
\text { attenuation }\end{array}$ & $\begin{array}{c}\text { Dominant } \\
\text { geomorphic } \\
\text { processes }\end{array}$ & $\begin{array}{l}\text { Observed/expected } \\
\text { causes for } \\
\text { disturbance }\end{array}$ & $\begin{array}{l}\text { Storm- } \\
\text { drain } \\
\text { outfalls }\end{array}$ & $\begin{array}{l}\text { Potential } \\
\text { geomorphic } \\
\text { changes }\end{array}$ & $\begin{array}{c}\text { Sensitivity } \\
\text { to } \\
\text { disturbance }\end{array}$ & $\begin{array}{c}\text { Geomorphic } \\
\text { segment } \\
\text { category }\end{array}$ \\
\hline BE & T, SOw & NO & EX, W, EBA, EBL & UP, UR, RO, HI & $\mathbf{N}$ & $\mathbf{Y}$ & LO & B \\
\hline BO & SOi, SOw & NO & I, W, EBA, EBF & UR, RO & $\mathrm{N}$ & Y & MOD & $\mathrm{U} 2$ \\
\hline BE & T, SOw & NO & $\mathrm{EX}, \mathbf{W}, \mathbf{E B A}, E B L$ & UP, UR, RO, HI & $\mathbf{N}$ & $\mathbf{Y}$ & LO & BT \\
\hline $\mathrm{BO} / \mathrm{CO}$ & $\mathrm{SOw}, \mathrm{SO}, \mathrm{SOb}$ & NO & W, EBA, $E B F, I, A$ & UR, RO & $\mathrm{Y}$ & $\mathrm{Y}$ & MOD & $\mathrm{T} 2$ \\
\hline $\mathrm{BO} / \mathrm{CO}$ & $\mathrm{T}, \mathrm{A}$ & NO & $\mathrm{EX}, A, W$ & UP, RO, CA & $\mathrm{Y}$ & $\mathrm{Y}$ & $\mathrm{LO}$ & L1 \\
\hline BE/BO & $\mathrm{T}, \mathrm{SOb}$ & NO & EBL, EX & UP, RO & $\mathbf{N}$ & $\mathbf{Y}$ & LO & B \\
\hline $\mathrm{CO}$ & $\mathrm{T}, \mathrm{SO} w, S O b$ & WE & $E B L, E B A, E X$ & UP, CL & $\mathrm{N}$ & $\mathrm{Y}$ & MOD & M2 \\
\hline $\mathrm{CO}$ & SOw, SOi & WE & $\mathrm{EX}, \mathrm{I}, \mathrm{W}, E B A$ & UR, RO & $\mathrm{N}$ & $\mathrm{Y}$ & MOD & $\mathrm{T} 2$ \\
\hline$B O$ & $\mathrm{~T}, \mathrm{SO} w, \mathrm{SOb}$ & WE & $\mathrm{EX}, E B A, E B F$ & UP, RO, CL & $\mathrm{N}$ & Y & MOD & B \\
\hline$B O$ & $\mathrm{SO} i, \mathrm{SO} w, \mathrm{SOb}$ & WE & $\mathrm{I}, \mathrm{A}, W, \mathrm{EBA}, E B L$ & UR, RO & $\mathrm{N}$ & $\mathrm{Y}$ & HI & $\mathrm{T} 2$ \\
\hline BO & none & NO & ST & NO & $\mathbf{N}$ & $\mathbf{N}$ & MOD & BT \\
\hline$G V$ & T, SOw, SIb, SIa & FP & $\mathrm{W}, I, \mathrm{EBA}, \mathrm{LM}, O S, A$ & UP, RO, GV, UR & $\mathrm{Y}$ & $\mathrm{Y}$ & HI & A \\
\hline $\mathrm{CO}$ & $\begin{array}{l}\text { SOi, SOb, SOw, } \\
\text { T, SIc }\end{array}$ & NO & $\begin{array}{l}\text { I, EBL, EBA, W, LM, } \\
\text { BF }\end{array}$ & UP, RO, GV & $\mathrm{N}$ & $\mathrm{Y}$ & HI & M1 \\
\hline$C O / G V$ & $\mathrm{SOb}, \mathrm{SOw}, \mathrm{T}$ & FP & $\begin{array}{l}\text { EBA, EBL, W, LM, } \\
\quad \text { BF }\end{array}$ & UP, RO, GV & $\mathrm{N}$ & $\mathrm{Y}$ & HI & M1 \\
\hline$G V$ & SOb, SOw, T & NO & $\begin{array}{l}\text { EBL, EBA, W, LM, } \\
\text { BF }\end{array}$ & UP, RO, GV, UR & $\mathrm{N}$ & $\mathrm{Y}$ & HI & A \\
\hline $\mathrm{CO}$ & T, SOw, SIc & NO & EBA, W, LM, BF & $\mathrm{RO}, \mathrm{GV}, \mathrm{UR}$ & $\mathrm{N}$ & $\mathrm{Y}$ & MOD & $\mathrm{U} 2$ \\
\hline $\mathrm{CO}$ & $\mathrm{T}, \mathrm{SOw}$ & NO & $E B A, W, I$ & $\mathrm{RO}, \mathrm{GV}$ & $\mathrm{N}$ & $\mathrm{Y}$ & MOD & $\mathrm{T} 2$ \\
\hline $\mathrm{BO}$ & T, SOw, SOi & NO & $I, E B A, W$ & $\mathrm{GV}, \mathrm{RO}, \mathrm{CL}$ & $\mathrm{N}$ & $\mathrm{Y}$ & MOD & BT \\
\hline$B E / B O$ & $\mathrm{SOi}$ & NO & $I, E X$ & $\mathrm{GV}, \mathrm{CL}$ & $\mathrm{N}$ & $\mathrm{Y}$ & LO & BT \\
\hline $\mathrm{CO}$ & $\mathrm{T}, \mathrm{SOw}$ & NO & $E B A, W$ & UP, RO, UR & $\mathrm{N}$ & $\mathrm{Y}$ & MOD & L2 \\
\hline$B E$ & $\mathrm{~T}, S O b$ & NO & $\mathrm{EX}, E B L$ & UP, RO & $\mathrm{N}$ & $\mathrm{Y}$ & $\mathrm{LO}$ & B \\
\hline$B O$ & $S O i, S O w, S O b$ & NO & $I, E B A, E B L$ & $\mathrm{CL}, \mathrm{RO}, \mathrm{HI}$ & $\mathrm{N}$ & $\mathrm{Y}$ & MOD & $\mathrm{U} 2$ \\
\hline $\mathrm{CO}$ & $\mathrm{T}, S O w$ & $N O$ & $E B A, W$ & $\mathrm{UP}, \mathrm{RO}, \mathrm{CL}$ & $\mathrm{N}$ & $\mathrm{Y}$ & MOD & L2 \\
\hline$B E$ & $\mathrm{~T}$ & $N O$ & $E X, E B L$ & UP, RO, HI & $\mathrm{N}$ & $\mathrm{Y}$ & LO & B \\
\hline
\end{tabular}


Table 5. Geomorphic characteristics of stream segments for Duluth-area streams, Minn., 2003-2004—Continued

[See table 6 for explanation of abbreviations. Stream segments are shown on figure 13. Data in italics are extrapolated from reach assessments of sites with the same segment category. Data in bold are rapid assessment or intensive survey sites. USGS, U.S. Geological Survey; ID, identification number; --, no detailed measurements taken; \%, percent; $>$, greater than; <, less than]

\begin{tabular}{|c|c|c|c|c|c|c|c|c|c|c|c|}
\hline $\begin{array}{c}\text { Segment } \\
\text { ID }\end{array}$ & Stream name & $\begin{array}{l}\text { Topographic } \\
\text { map }\end{array}$ & $\begin{array}{l}\text { USGS } \\
\text { reach } \\
\text { ID }\end{array}$ & $\begin{array}{c}\text { Drainage- } \\
\text { network } \\
\text { position }\end{array}$ & $\begin{array}{c}\text { Slope } \\
\%\end{array}$ & $\begin{array}{l}\text { Valley } \\
\text { type }\end{array}$ & $\begin{array}{c}\text { Bedrock } \\
\text { type }\end{array}$ & $\begin{array}{c}\text { Glacial } \\
\text { landform }\end{array}$ & $\begin{array}{l}\text { Dominant } \\
\text { land cover }\end{array}$ & $\begin{array}{l}\text { Inter- } \\
\text { mittent }\end{array}$ & $\begin{array}{c}\text { Channel } \\
\text { type }\end{array}$ \\
\hline sv4 & $\begin{array}{l}\text { Lenroot St Creek, } \\
\text { Gogebic St Creek, } \\
\text { 84th Ave W } \\
\text { Creek, 85th Ave } \\
\text { W Creek }\end{array}$ & West Duluth & -- & MM & $>8$ & $\mathrm{C}$ & GAB & $\mathrm{BED} / \mathrm{SH}$ & $\mathrm{F}$ & $\mathrm{N}$ & $\mathrm{BE}$ \\
\hline sv5 & $\begin{array}{l}\text { Lenroot St Creek, } \\
\text { Gogebic St Creek, } \\
\text { 84th Ave W } \\
\text { Creek, 85th Ave } \\
\text { W Creek }\end{array}$ & West Duluth & -- & UM & $>4-8$ & $\mathrm{C}$ & GAB & $\mathrm{BED} / \mathrm{SH}$ & $\mathrm{F}$ & $\mathrm{N}$ & $\mathrm{CA} / \mathrm{BE}$ \\
\hline t1 & Tischer Creek & Duluth & -- & LM & $>4-8$ & $\mathrm{E}$ & IN & BED/TP & $\mathrm{U}$ & $\mathrm{N}$ & $\mathrm{BE}$ \\
\hline $\mathrm{t} 2$ & Tischer Creek & Duluth & -- & MM & $>1-2$ & $\mathrm{~N}$ & IN & BED & $\mathrm{U}$ & $\mathrm{N}$ & $P R$ \\
\hline $\mathrm{t} 3$ & Tischer Creek & Duluth & -- & MM & $>1-2$ & $\mathrm{C}$ & GAB & BED & $\mathrm{U}$ & $\mathrm{N}$ & $P R$ \\
\hline t4 & Tischer Creek & Duluth & -- & MM & $0.3-1$ & $\mathrm{~N}$ & GAB & BED & S & $\mathrm{N}$ & AR \\
\hline t5 & Tischer Creek & Duluth & -- & MM & $>2-4$ & $\mathrm{C}$ & GAB & BED & $\mathrm{U}$ & $\mathrm{N}$ & $P B$ \\
\hline t6 & Tischer Creek & Duluth & -- & $\mathrm{UM}$ & $0.3-1$ & $\mathrm{~N}$ & GAB & BED & $\mathrm{F} / \mathrm{S} / \mathrm{U}$ & $\mathrm{N}$ & AR \\
\hline t7 & Tischer Creek & Duluth & -- & MT & $>1-2$ & $\mathrm{C}$ & GAB & BED & $\mathrm{U} / \mathrm{F}$ & $\mathrm{Y}$ & $P B$ \\
\hline t8 & Tischer Creek & Duluth & -- & MT & $>1-2$ & E & VOL & BED & $\mathrm{U} / \mathrm{F}$ & $\mathrm{N}$ & $A R / P R$ \\
\hline t9 & Tischer Creek & Duluth & 10 & LM & $>8$ & $\mathbf{E}$ & IN & BED & $\mathbf{U}$ & $\mathbf{N}$ & $\mathbf{B E}$ \\
\hline u1 & 34th Ave E Creek & Duluth & -- & LM & $>4-8$ & $\mathrm{E}$ & IN & BED & $\mathrm{U}$ & $\mathrm{N}$ & $\mathrm{BE}$ \\
\hline u2 & 38th Ave E Creek & Duluth & -- & LM & $>4-8$ & $\mathrm{C}$ & VOL & BED & $\mathrm{U}$ & $\mathrm{N}$ & $\mathrm{BE}$ \\
\hline u3 & 43rd Ave E Creek & Duluth & -- & LM & $>4-8$ & $\mathrm{C}$ & VOL & BED & $\mathrm{U}$ & $\mathrm{N}$ & $\mathrm{BE}$ \\
\hline u4 & 43rd Ave E Creek & Duluth & -- & MM & $>8$ & $\mathrm{E}$ & VOL & BED & $\mathrm{U}$ & $\mathrm{N}$ & $\mathrm{BE}$ \\
\hline u5 & 43rd Ave E Creek & Duluth & -- & UM & $>2-4$ & $\mathrm{C}$ & VOL/IN & BED & $\mathrm{U}$ & $\mathrm{N}$ & AR \\
\hline u6 & 50th Ave E Creek & Duluth & -- & MT & $>2-4$ & $\mathrm{C}$ & VOL & BED & $\mathrm{U}$ & $\mathrm{Y}$ & $\mathrm{CA}$ \\
\hline u7 & 40th Ave E Creek & Duluth & -- & UT & $>4-8$ & $\mathrm{C}$ & VOL/IN & BED & $\mathrm{U}$ & $\mathrm{Y}$ & $\mathrm{CO}$ \\
\hline
\end{tabular}




\begin{tabular}{ccccccccc}
\hline $\begin{array}{c}\text { Dominant } \\
\text { substrate }\end{array}$ & $\begin{array}{c}\text { Mode of } \\
\text { sediment transport }\end{array}$ & $\begin{array}{c}\text { Flood } \\
\text { attenuation }\end{array}$ & $\begin{array}{c}\text { Dominant } \\
\text { geomorphic } \\
\text { processes }\end{array}$ & $\begin{array}{c}\text { Observed/expected } \\
\text { causes for } \\
\text { disturbance }\end{array}$ & $\begin{array}{c}\text { Storm- } \\
\text { drain } \\
\text { outfalls }\end{array}$ & $\begin{array}{c}\text { Potential } \\
\text { geomorphic } \\
\text { changes }\end{array}$ & $\begin{array}{c}\text { Sensitivity } \\
\text { to } \\
\text { disturbance }\end{array}$ & $\begin{array}{c}\text { Geomorphic } \\
\text { segment } \\
\text { category }\end{array}$ \\
\hline $\mathrm{BE}$ & $\mathrm{T}$ & $\mathrm{NO}$ & $E X, E B L$ & $\mathrm{UP}, \mathrm{RO}, \mathrm{HI}$ & $\mathrm{N}$ & $\mathrm{Y}$ & $\mathrm{LO}$ & $\mathrm{B}$
\end{tabular}

\begin{tabular}{|c|c|c|c|c|c|c|c|c|}
\hline $\mathrm{BE} / \mathrm{BO}$ & SOw & NO & $S T$ & $\mathrm{RO}, \mathrm{CL}, \mathrm{HI}$ & $\mathrm{N}$ & $\mathrm{Y}$ & LO & B \\
\hline $\mathrm{BE} / \mathrm{BO}$ & $\mathrm{T}, \mathrm{SOb}$ & NO & EX, EBL & HI, UP, RO & $\mathrm{Y}$ & $\mathrm{Y}$ & LO & $\mathrm{B}$ \\
\hline$G V$ & SOi, SOw, T & NO & W, EBA, LM & UP, UR, RO & $\mathrm{Y}$ & $\mathrm{Y}$ & HI & M1 \\
\hline$G V$ & SOi, SOw, SIc, T & NO & W, EBA, LM, BF & UP, UR, RO & $\mathrm{Y}$ & $\mathrm{Y}$ & HI & M1 \\
\hline FINES & SIa & FP/WE & A & UP, UR, CA & $\mathrm{Y}$ & $\mathrm{Y}$ & LO & M.3 \\
\hline $\mathrm{BO} / \mathrm{CO}$ & SOi, SOw, T, SIc & NO & $\mathrm{W}, \mathrm{EBA}, I, \mathrm{LM}, \mathrm{BF}$ & UP, UR, CL & $\mathrm{Y}$ & $\mathrm{Y}$ & HI & M2 \\
\hline GV/FINES & SOi, SOw & WE/NO & W, EBA, A, I & CA, UR, RO, CL & $\mathrm{N}$ & $\mathrm{Y}$ & MOD & W.3 \\
\hline $\mathrm{CO}$ & SOi, SOw, SIc & NO & W, EBA, I, BF & $\mathrm{CL}, \mathrm{CA}, \mathrm{UR}$ & $\mathrm{Y}$ & $\mathrm{Y}$ & HI & $\mathrm{T} 1$ \\
\hline GV/CO & SOi, SOw, T & NO & W, EBA, I & $\mathrm{CL}, \mathrm{A}, \mathrm{UR}, \mathrm{RO}$ & $\mathrm{Y}$ & $\mathrm{Y}$ & HI & $\mathrm{T} 1$ \\
\hline $\mathrm{BE} / \mathrm{BO}$ & $\mathrm{T}, \mathrm{SOb}$ & No & EX, EBL & HI, UP & $\mathbf{Y}$ & $\mathbf{Y}$ & LO & B \\
\hline $\mathrm{BE} / \mathrm{BO}$ & T, SOi, SOb, SOw & NO & EX, I, EBL & UR & $\mathrm{Y}$ & $\mathrm{Y}$ & MOD & B \\
\hline $\mathrm{BE} / \mathrm{BO}$ & T, SOi, SOb, SOw & NO & EX, I, EBL & UR & $\mathrm{Y}$ & $\mathrm{Y}$ & LO & $\mathrm{B}$ \\
\hline $\mathrm{BE} / \mathrm{BO}$ & T, SOi, SOb, SOw & NO & EX, I, EBL & UR & $\mathrm{Y}$ & $\mathrm{Y}$ & LO & B \\
\hline $\mathrm{BE} / \mathrm{BO}$ & T, SOi, SOb, SOw & NO & EX, I, EBL & UR & $\mathrm{Y}$ & Y & LO & B \\
\hline $\mathrm{CO}$ & SOi, SOw & $F P$ & W, I, EBL, A & CA, UR, CL & $\mathrm{Y}$ & $\mathrm{Y}$ & MOD & $\mathrm{U} 2$ \\
\hline$B O$ & T, SOi, SOb, SOw & NO & $\mathrm{W}, E X, \mathrm{I}$ & UR & $\mathrm{N}$ & $\mathrm{Y}$ & MOD & $\mathrm{T} 2$ \\
\hline $\mathrm{BE} / \mathrm{BO}$ & T, SOi, SOb, SOw & NO & EX, I, LM, W & $\mathrm{UR}, \mathrm{CL}$ & $\mathrm{Y}$ & $\mathrm{Y}$ & MOD & BT \\
\hline
\end{tabular}


Table 6. Explanation of abbreviations used in tables for segment and reach characteristics in this study of Duluth-area streams, Minn.

\begin{tabular}{ll}
\hline & \multicolumn{1}{c}{ Drainage-network position } \\
\hline UM & Upper main stem \\
MM & Middle main stem \\
LM & Lower main stem \\
UT & Upper main-stem tributary \\
MT & Middle main-stem tributary \\
LT & lower main-stem tributary \\
\end{tabular}

\begin{tabular}{ll}
\hline N & \multicolumn{1}{c}{ Valley type/stream meandering } \\
C & $\begin{array}{c}\text { No valley development, stream unconfined and flows } \\
\text { Confined, V-shaped valleys with no flood plain, bed- } \\
\text { rock, or colluvial }\end{array}$ \\
E & $\begin{array}{c}\text { Unconfined valley, entrenched meanders; stream me- } \\
\text { anders follow valley course, affected by geologic } \\
\text { setting; some flood plain }\end{array}$ \\
& $\begin{array}{l}\text { Unconfined valley with alluvial meanders, stream } \\
\text { meanders reflect fluvial processes; valley wider }\end{array}$ \\
& $\quad$ than meander belt, wide flood plain \\
B & Confined valley formed in bedrock, no flood plain \\
& \multicolumn{1}{c}{ Dominant bedrock type for segment } \\
\hline SED & Slate/sedimentary \\
GAB & Duluth gabbro complex \\
VOL & Felsic and mafic volcanics \\
IN & Intrusions \\
BUR & Bedrock buried by glacial deposits \\
& \\
\hline & $\quad$ Glacial landform/deposits \\
\hline SD & Supraglacial drift \\
OW & Outwash \\
IC & Ice contact \\
LA & Glacial lake \\
SH & Post-glacial shoreline/beach \\
BED & Bedrock surface \\
Till plain & Undifferentiated \\
\hline &
\end{tabular}

\begin{tabular}{ll}
\hline \multicolumn{1}{c}{ Dominant watershed land cover } \\
\hline U & Urban \\
D & Developing urban \\
A & Agriculture \\
F & Forest \\
W & Wetland \\
G & Grass \\
S & Shrubland \\
R & Roads \\
& \\
\hline & \\
\hline CO & Colluvial \\
BE & Bedrock \\
CA & Cascade \\
SP & Step-pool \\
PB & Plane-bed \\
PR & Pool-riffle \\
AR & Artificial \\
WE & Wetland \\
& \\
\hline & \\
\hline BD & Bedrock \\
BO & Boulder \\
CO & Cobble \\
GV & Gravel \\
SA & Sand \\
FINES & Fines/Organic debris \\
AR & Artificial - concrete and others \\
\hline &
\end{tabular}


Table 6. Explanation of abbreviations used in tables for segment and reach characteristics in this study of Duluth-area streams, Minn.-Continued.

\begin{tabular}{ll}
\hline & \multicolumn{1}{c}{ Mode of sediment transport } \\
\hline SOi & Source-incision \\
SOb & Source-bluff erosion \\
SOw & Source-bank erosion, widening \\
T & Transfer \\
SIc & Sink—-lateral and mid-channel sand/gravel bars \\
SIb & Sink—overbank sedimentation \\
SIa & Sink—aggradation \\
SIp & Sink—-fines in pools \\
\end{tabular}

\section{Flood attenuation}

\begin{tabular}{ll}
\hline NO & None \\
FP & Flood-plain attenuation \\
WE & Wetland storage
\end{tabular}

\begin{tabular}{ll}
\hline & \multicolumn{1}{c}{ Dominant geomorphic processes } \\
\hline H & Headcutting \\
I & Incision \\
EBL & Bluff/terrace erosion \\
EBA & Bank erosion \\
W & Widening \\
LM & Lateral migration \\
OS & Overbank sedimentation/levee formation \\
BF & Mid-channel/lateral bar formation \\
A & Aggradation \\
EX & Expansion of flow area \\
ST & Stable \\
& \\
\hline & $\quad$ Causes for geomorphic disturbance \\
\hline CL & Logging, agriculture, clearing \\
UP & Upstream changes in sediment/water inputs \\
UR & Urban development \\
CA & Man-made channel alterations \\
RO & Road drainage \\
DI & Ditching \\
HI & Hiking trails \\
GV & Gravel pits \\
NO & None \\
\hline
\end{tabular}

\begin{tabular}{|c|c|}
\hline \multicolumn{2}{|r|}{ Potential changes in sediment/water inputs } \\
\hline $\mathrm{Y}$ & Yes \\
\hline $\mathrm{N}$ & No \\
\hline \multicolumn{2}{|r|}{ Potential sensitivity to change } \\
\hline LO & $\begin{array}{l}\text { Low-little or no change in overall channel morphol- } \\
\text { ogy expected }\end{array}$ \\
\hline MO & $\begin{array}{l}\text { Moderate-minor changes in substrate size, width, } \\
\text { pool depth }\end{array}$ \\
\hline HI & $\begin{array}{l}\text { High-substantial, readily detectable changes in } \\
\text { channel morphology with a change in an important } \\
\text { factor }\end{array}$ \\
\hline
\end{tabular}




\section{Reach Characteristics and Processes}

Reach characteristics for the 48 rapid assessment and intensive survey sites are summarized in table 7 . The table contains a list of the dominant geomorphic processes at each site; modes of sediment erosion, transport, and deposition; geomorphic sensitivity to change; and remarks on pool and LWD source and function. The rapid assessment and intensive survey sites were in a variety of segment categories of slope, drainage-network position, and valley types (table 5). All but four sites were on main stems and all but one had perennial streamflow.

The intensive sites mainly were in two of the largest watersheds in the Duluth area-Miller Creek $\left(9.3 \mathrm{mi}^{2}\right)$ and Lester River $\left(36 \mathrm{mi}^{2}\right)$. This design allowed for an understanding of the downstream progression of geomorphic conditions and processes, including linkages among sediment erosion, transport, and deposition. Miller Creek was chosen for more detailed study because of past and ongoing urban development in its upper watershed and erosion-resistant gabbro bedrock. The Lester River watershed had mainly rural land and contained volcanic and intrusive rocks. An intensive site was measured along Mission Creek in a transitional reach near a glacial lake shoreline between glacial deposits and bedrock (site 1), which has urban development upstream and future potential for valley side failures. An intensive site also was in a transitional reach along Chester Creek (site 43) between glacial deposits and bedrock. Both of these sites are in reaches near the upstream boundary between glacial deposits, glacial lake shorelines, and bedrock. These two sites had similar geologic settings to site 23 on the Lester River and site 34 on Miller Creek (figs. 1, 4, and 5).

Most of the channel types described by Montgomery and Buffington (1998) were present in Duluth-area streams except for dune-ripple types (fig. 10, table 8). Fitting possible channel characteristics into categories for the segments was sometimes difficult for reaches in slope transition zones where channel conditions changed rapidly. Also, it was difficult to distinguish whether some streams were flowing on alluvium, colluvium, or glacial outwash deposits. Wetland channels and artificial ditches most commonly had slopes of less than 0.3 percent but some had slopes of 0.3 to 1 percent. Wetland ditches contained fine-grained deposits of organic-rich material during low flow that was most likely transported downstream during runoff events. Plane-bed channels were in middle main-stem transition areas between glacial deposits and exposed bedrock, in lower main-stem transition areas where streams flowed from steep bedrock to gentle till

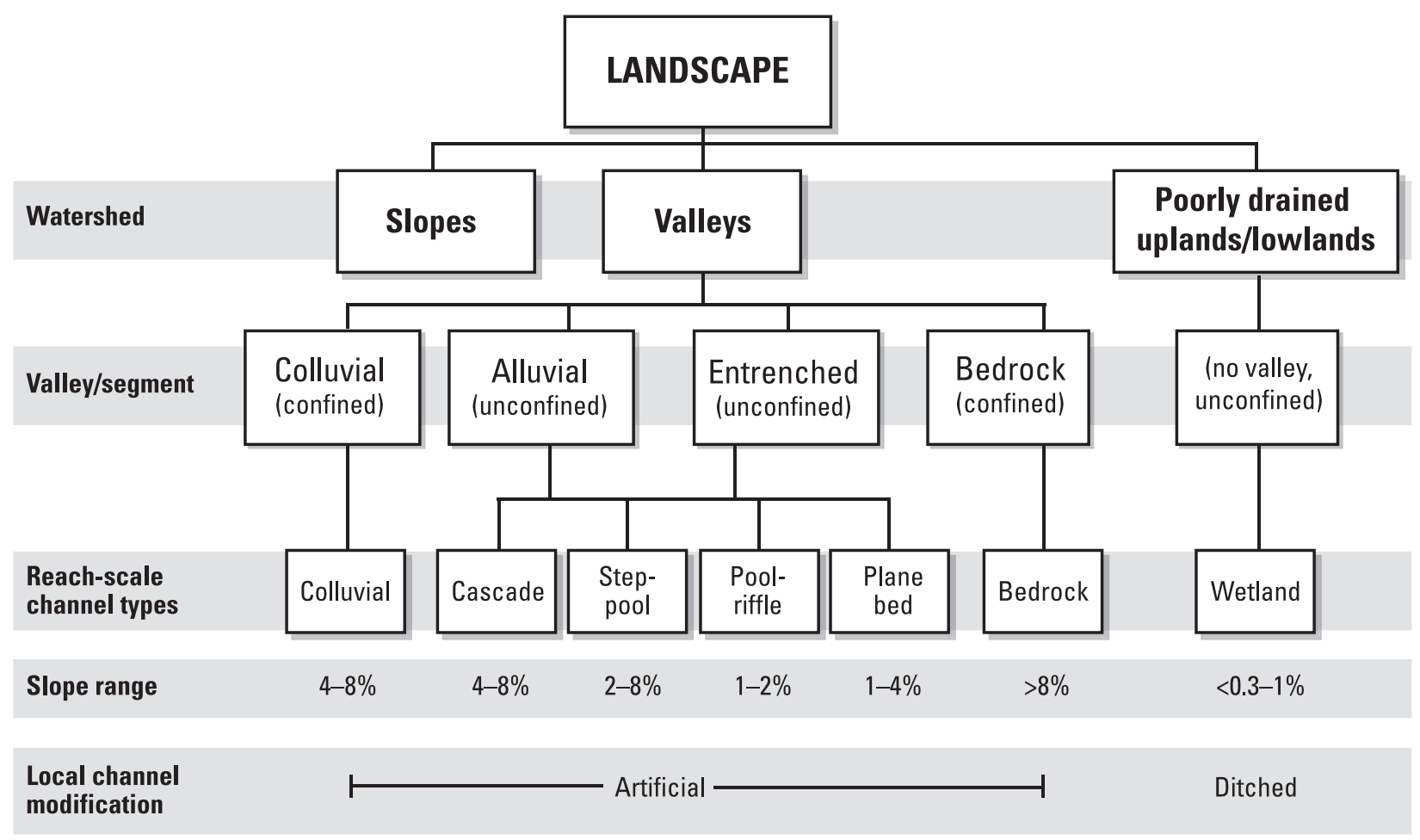

Figure 10. Hierarchical classification of channel types in the Duluth, Minn., area (modified from Montgomery and Buffington, 1993; 1998). (\%, percent; $>$, greater than; $<$, less than) 
plain or landfill. Plane-bed channels generally had slopes from about 1 to 4 percent. Pool-riffle channels had slopes of about 1 to 2 percent and generally were in streams intersecting glacial deposits. Step-pool channels were mainly in middle main stems with slopes greater than 2 percent where glacial deposits transitioned to bedrock at the land surface. At slopes greater than 4 percent, channel types on main stems were cascade or bedrock and on tributaries were colluvial or bedrock.

Geomorphic characteristics plotted on longitudinal profiles for Lester River (fig. 11A) and Miller Creek (fig. $11 B$ ) illustrate how segment and reach characteristics progressively change from upstream to downstream. In the Lester River watershed, the upper main stem flows through wetlands, with gentle slopes and ditches (site 25). Progressing downstream at site 24 , the slope remains gentle as the stream flows across supraglacial drift. At site 24 , there is no valley, runoff and sediment inputs are low, and LWD is rare. Farther downstream, the entrenched valley is cut into glacial outwash and ice contact deposits and the slope increases (site 30). Evidence of valley side failures or landslides is present (accentuated by the narrow valley and erodible glacial deposits) and bank erosion is common along straight stretches and bends, indicating channel widening. It is likely that, at about this point along the main stem, tributaries become sediment sources to the main stem. Overbank sedimentation is occurring on the flood plain. Farther downstream at site 23 (fig. 1), the slope lessens where the bedrock changes from a gabbro intrusion to volcanic rocks. Sediment deposition in the channel and in overbank areas is more common than at site 30, along with LWD jams. The channel shows signs of widening and bank erosion is common but there is less lateral migration and valley side failures are less common than upstream. At site 20, the slope increases where the stream intersects a gabbro intrusion and the channel type is step-pool. Farther downstream at site 31, the steep bedrock channel shows scour along channel margins, an indication of channel expansion. Bank erosion is more likely to occur where banks are composed of alluvium instead of bedrock or colluvium. The reach at site 31 functions as a transfer zone, and sediment and LWD are transported through the reach and downstream. There is little or no overbank sedimentation. LWD originating from tree-lined banks or valley side slopes is transported downstream. Falls or rapids are at intersections with erosion-resistant felsic rocks, or rocks with less jointing or air pockets, whereas pools or cascades are at intersections with mafic rocks, which are less resistant to erosion. Pools are free-formed below falls; pool levels are sometimes raised by human modification (stacking rocks at the lower end) for swimming. Bluff and gully erosion is common along hiking trails and near storm drain outfalls that run along the valley top because they tend to concentrate runoff. Landslides also are frequent where runoff becomes concentrated. Feeder tributaries in this reach are prone to gully erosion and provide local sources of sediment to the main stem.

Similar to Lester River, the upper main stem of Miller Creek begins with wetland ditches with gentle slopes (site 33 , fig. 11B). Site 34 is downstream of an area with extensive shopping development and upstream of the transition from glacial deposits to bedrock at the land surface. The channel type at site 34 is pool-riffle with evidence for recent (within 5 years) channel widening, avulsion (sudden channel movement), and flood scour. The flood plain has about $1.5 \mathrm{ft}$ of post-settlement alluvium. LWD is common and provides some habitat and pools. Downstream, in the steep reach affected by the Duluth-complex gabbro rocks (site 44), the cascade-type channel shows signs of channel expansion in areas with no flood plain or widening in areas with flood plain. There is minimal to no overbank sedimentation through the reach at site 34 . This site functions as a transfer zone for upstream sources of sediment and upstream or bank-derived LWD. Site 37 downstream is an example of a bedrock channel with a slope of greater than 8 percent. Similar to Lester River, bedrock channels on Miller Creek show evidence of channel expansion (scoured bedrock along channel margins and trees toppling into the channel) and sediment inputs from landslides and gully development along the tops of valley sides related to storm drains and hiking trails. Downstream of the bedrock-controlled zone, the channel is an artificial plane-bed at site 36 .

No intensive sites were on tributaries, but observations and photographs from tributaries or small main stems with rapid assessments indicated that middle and lower tributaries with relatively steep slopes had colluvial, cascade, or bedrock channel types (fig. 12). For example, site 46 on Coffee Creek (considered a tributary to the lower main stem of Miller Creek), was ephemeral and had a mix of colluvial/bedrock channel types (fig. 12A). Tributaries to Merritt, Stewart, and Keene Creeks were perennial (figs. $12 B-D$ ). The Stewart Creek tributary (site 2) had a small colluvial channel that was hidden by vegetation. The Merritt Creek tributary (site 47) had a bedrock channel. The Keene Creek tributary (site 5) had a cascade channel. The amount of scour in the channels was attributed to upstream land cover and effects on runoff and flood peaks. Coffee Creek had an urban watershed; Stewart Creek had a forested watershed. Merritt Creek was forested at the site but 
Table 7. Reach characteristics at rapid assessment and intensive survey field sites for Duluth-area streams, Minn., 2003-2004.

[USGS, U.S. Geological Survey; mi, mile; ft, foot; LWD, large woody debris; cfs, cubic foot per second; ’, degree; '. minute; ", second; mi², square mile; $\%$, percent; gv, gravel; co, cobble; FP, flood plain; m, medium; c, coarse; ns, not sampled; bf, bankfull; w, width; d, depth; bo, boulder; ds, downstream; us, upstream; , approximately; sed, sediment; <, less than; occas., occasional; ns, not surveyed; accum, accumulation; trib, tributary]

\begin{tabular}{|c|c|c|c|c|c|c|c|}
\hline $\begin{array}{l}\text { USGS } \\
\text { field } \\
\text { number }\end{array}$ & $\begin{array}{l}\text { Stream } \\
\text { name }\end{array}$ & Latitude & Longitude & $\begin{array}{l}\text { Drainage } \\
\text { area } \\
\left(\mathrm{mi}^{2}\right)\end{array}$ & $\begin{array}{l}\text { Measured } \\
\text { reach } \\
\text { slope }(\%)\end{array}$ & Channel geomorphic setting & Pool notes \\
\hline 1 & $\begin{array}{l}\text { Mission Creek at } \\
\text { Stenman Rd. }\end{array}$ & $46^{\circ} 41^{\prime} 43^{\prime \prime}$ & $92^{\circ} 18^{\prime} 42^{\prime \prime}$ & 2.12 & 1.6 & $\begin{array}{l}\text { Slightly incised? Widening. Large gv/co load } \\
\text { carried by stream, bars mobilized during } \\
\text { floods, accumulations around LWD; large } \\
\text { trees on bank falling in. Boulder zones may } \\
\text { be related to glacial lake shoreline or road } \\
\text { construction. }\end{array}$ & $\begin{array}{l}\text { Pool habitat caused } \\
\text { by three main } \\
\text { sources. }\end{array}$ \\
\hline 2 & $\begin{array}{r}\text { Stewart Creek } \\
\text { Tributary at } \\
\text { Skyline Rd. }\end{array}$ & $46^{\circ} 42^{\prime} 07^{\prime \prime}$ & $92^{\circ} 13^{\prime} 43^{\prime \prime}$ & ns & no & $\begin{array}{l}\text { Bedrock controlled channel, no evidence of } \\
\text { incision or widening. }\end{array}$ & ns \\
\hline 3 & $\begin{array}{l}\text { Stewart Creek at } \\
\text { Skyline Rd. }\end{array}$ & $46^{\circ} 42^{\prime} 13^{\prime \prime}$ & $92^{\circ} 13^{\prime} 41^{\prime \prime}$ & $\mathrm{ns}$ & no & $\begin{array}{l}\text { Bedrock controlled channel, increases in bf } \\
\mathrm{w} \text { and d due to increased flow, no apparent } \\
\text { incision or widening. }\end{array}$ & $\mathrm{ns}$ \\
\hline 4 & $\begin{array}{l}\text { Knowlton Creek } \\
\text { at South } \\
\text { Boundary Ac- } \\
\text { cess Rd. }\end{array}$ & $46^{\circ} 43^{\prime} 30^{\prime \prime}$ & $92^{\circ} 13 ' 20^{\prime \prime}$ & ns & no & $\begin{array}{l}\text { Bedrock controlled channel, increases in bf } \\
\text { w and d due to increased flow, no incision } \\
\text { or widening, human hardening of channel } \\
\text { location. }\end{array}$ & $\begin{array}{l}\text { Pools caused by } \\
\text { scour at base of } \\
\text { falls. }\end{array}$ \\
\hline 5 & $\begin{array}{l}\text { Keene Creek } \\
\text { Tributary at } \\
\text { Skyline Rd./ } \\
\text { Getchell Rd. }\end{array}$ & $46^{\circ} 45^{\prime} 01^{\prime \prime}$ & $92^{\circ} 11^{\prime} 17^{\prime \prime}$ & $\mathrm{ns}$ & no & $\begin{array}{l}\text { Rocks scoured, bo/gr banks are stable, road } \\
\text { runoff a problem from Skyline Rd. }\end{array}$ & Pools rare. \\
\hline 6 & $\begin{array}{l}\text { Keene Creek at } \\
\text { Skyline Rd. }\end{array}$ & $46^{\circ} 45^{\prime} 21^{\prime \prime}$ & $92^{\circ} 11^{\prime} 21 "$ & ns & no & ns & ns \\
\hline 7 & $\begin{array}{l}\text { Miller Creek at } \\
\text { Skyline Rd. }\end{array}$ & $46^{\circ} 46^{\prime} 25^{\prime \prime}$ & $92^{\circ} 08^{\prime} 35^{\prime \prime}$ & ns & no & $\mathrm{ns}$ & $\mathrm{ns}$ \\
\hline 8 & $\begin{array}{l}\text { Buckingham } \\
\text { Creek at } \\
\text { Skyline Rd. }\end{array}$ & $46^{\circ} 46^{\prime} 43^{\prime \prime}$ & $92^{\circ} 07^{\prime} 23^{\prime \prime}$ & ns & no & $\mathrm{ns}$ & ns \\
\hline 9 & $\begin{array}{l}\text { Chester Creek at } \\
\text { Skyline Rd. }\end{array}$ & $46^{\circ} 48^{\prime} 46^{\prime \prime}$ & $92^{\circ} 05^{\prime} 30^{\prime \prime}$ & ns & no & $\mathrm{ns}$ & ns \\
\hline 10 & $\begin{array}{l}\text { Tischer Cr. at } \\
\text { Skyline Rd./ } \\
\text { Vermillion Rd. }\end{array}$ & $46^{\circ} 50^{\prime} 48^{\prime \prime}$ & $92^{\circ} 03^{\prime} 57^{\prime \prime}$ & ns & no & $\mathrm{ns}$ & ns \\
\hline 11 & $\begin{array}{l}\text { Amity Creek at } \\
\text { Occidnetal } \\
\text { Rd., Lester } \\
\text { Park }\end{array}$ & $46^{\circ} 50^{\prime} 39^{\prime \prime}$ & $92^{\circ} 00^{\prime} 37^{\prime \prime}$ & ns & no & $\begin{array}{l}\text { Bedrock channel, rocks scoured, evidence of } \\
\text { increased bankfull width and depth. }\end{array}$ & $\begin{array}{l}\text { Pools caused by } \\
\text { scour at base of } \\
\text { falls. }\end{array}$ \\
\hline 12 & $\begin{array}{l}\text { Amity Creek at } \\
\text { Occidental } \\
\text { Rd., Amity } \\
\text { Creek Park, } \\
\text { \# } 1\end{array}$ & $46^{\circ} 50^{\prime} 56^{\prime \prime}$ & $92^{\circ} 00^{\prime} 41^{\prime \prime}$ & ns & no & Bedrock controlled channel. & ns \\
\hline 13 & $\begin{array}{l}\text { Amity Creek at } \\
\text { Occidental } \\
\text { Rd., Amity } \\
\text { Creek Park, } \\
\text { \# } 2\end{array}$ & $46^{\circ} 51^{\prime} 14^{\prime \prime}$ & $92^{\circ} 00^{\prime} 41^{\prime \prime}$ & ns & no & Bedrock controlled channel. & ns \\
\hline
\end{tabular}




\begin{tabular}{|c|c|c|c|c|}
\hline LWD notes & $\begin{array}{c}\text { Overbank } \\
\text { sedimentation }\end{array}$ & $\begin{array}{l}\text { Estimate of } \\
\text { streamflow } \\
\quad \text { (cfs) }\end{array}$ & Sensitivity to change & Additional notes \\
\hline $\begin{array}{l}\text { Large trees in flood plain are } \\
\text { source for LWD. Fallen trees } \\
\text { starting to hang up on banks } \\
\text { above water surface. Channel } \\
\text { avulsion occurring, LWD may } \\
\text { not stay in place. }\end{array}$ & $\begin{array}{l}\text { FP on inside of bend has } \\
1-1.5 \mathrm{ft} \text { organic rich } \mathrm{m} / \mathrm{c} \\
\text { sandy loam to sand over } \\
\text { gv/co. The surficial A } \\
\text { horizon is about } 1 \mathrm{ft} \text { thick. } \\
\text { Sediment accum. is slow. }\end{array}$ & 0.5 & $\begin{array}{l}\text { High-increases in runoff or sediment } \\
\text { loads will affect habitat. Reach affected } \\
\text { by Highway } 35 \text { runoff. Potential to } \\
\text { migrate into valley sides. Potential loss } \\
\text { of LWD function and pools. Sensitive to } \\
\text { road runoff, gullying from road runoff, } \\
\text { ditch construction, and landslides. }\end{array}$ & $\begin{array}{l}\text { Anecdotal evidence that the } \\
\text { reach previously had brook } \\
\text { trout, which disappeared after } \\
\text { Highway } 35 \text { was built. Culvert } \\
\text { at Stenman Rd. blocks fish } \\
\text { passage. }\end{array}$ \\
\hline ns & ns & $<.25$ & $\begin{array}{l}\text { Sensitive to road runoff-related } \\
\text { landslides, and ditching. }\end{array}$ & $\begin{array}{l}\text { Boulder bottom, moss-covered } \\
\text { rocks, minimal bank erosion, in- } \\
\text { cipient channel flowing around } \\
\text { boulders, occas. exposed roots. }\end{array}$ \\
\hline $\mathrm{ns}$ & ns & $2-3$ & $\begin{array}{l}\text { Sensitive to road runoff-related } \\
\text { landslides, ditch construction. }\end{array}$ & $\begin{array}{l}\text { Air photos show landslides in } \\
\text { the vicinity. }\end{array}$ \\
\hline $\begin{array}{l}\text { LWD absent, any wood would } \\
\text { have to come from us and is } \\
\text { likely transported through this } \\
\text { reach. }\end{array}$ & ns & $.25-.5$ & $\begin{array}{l}\text { Sensitivity to road runoff, increases in } \\
\text { bankfull width/depth. }\end{array}$ & $\begin{array}{l}\text { S. Boundary Rd. culvert blocks } \\
\text { fish passage and is grade con- } \\
\text { trol, site near transition in slope. }\end{array}$ \\
\hline LWD absent. & ns & .5 & $\begin{array}{l}\text { Sensitivity to road runoff, increases in } \\
\text { bankfull width/depth. }\end{array}$ & $\begin{array}{l}\text { Culvert blocks fish passage, } \\
\text { marked as ephemeral on topo- } \\
\text { graphic map but has base flow. }\end{array}$ \\
\hline ns & ns & ns & $\mathrm{ns}$ & none \\
\hline ns & ns & ns & ns & none \\
\hline $\mathrm{ns}$ & ns & ns & $\mathrm{ns}$ & $\begin{array}{l}\text { Between Twin Lakes. Quarries } \\
\text { common. Golf course us. Upper } \\
\text { valley runs between ridges of } \\
\text { gabbro. }\end{array}$ \\
\hline $\mathrm{ns}$ & ns & ns & $\mathrm{ns}$ & Narrow, confined valley in park. \\
\hline ns & ns & ns & $\mathrm{ns}$ & none \\
\hline $\begin{array}{l}\text { LWD rare, most transported } \\
\text { through reach. }\end{array}$ & None, outcrop. & 4 & $\begin{array}{l}\text { Sensitivity to road runoff, increases in } \\
\text { bankfull width/depth, local erosion from } \\
\text { hiking trail. }\end{array}$ & $\begin{array}{l}\text { Lester Park is heavily used, } \\
\text { erosion coincident with trails. }\end{array}$ \\
\hline ns & ns & ns & ns & none \\
\hline ns & ns & ns & $\mathrm{ns}$ & none \\
\hline
\end{tabular}


Table 7. Reach characteristics at rapid assessment and intensive survey field sites for Duluth-area streams, Minn., 2003-2004 -Continued.

[USGS, U.S. Geological Survey; mi, mile; ft, foot; LWD, large woody debris; cfs, cubic foot per second; ${ }^{\circ}$, degree; '. minute; ", second; mi², square mile; $\%$, percent; gv, gravel; co, cobble; FP, flood plain; m, medium; c, coarse; ns, not sampled; bf, bankfull; w, width; d, depth; bo, boulder; ds, downstream; us, upstream; , approximately; sed, sediment; <, less than; occas., occasional; ns, not surveyed; accum, accumulation; trib, tributary]

\begin{tabular}{|c|c|c|c|c|c|c|c|}
\hline $\begin{array}{l}\text { USGS } \\
\text { field } \\
\text { number }\end{array}$ & $\begin{array}{c}\text { Stream } \\
\text { name }\end{array}$ & Latitude & Longitude & $\begin{array}{l}\text { Drainage } \\
\text { area } \\
\left(\mathrm{mi}^{2}\right)\end{array}$ & $\begin{array}{l}\text { Measured } \\
\text { reach } \\
\text { slope }(\%)\end{array}$ & Channel geomorphic setting & Pool notes \\
\hline 14 & $\begin{array}{l}\text { Amity Creek at } \\
\text { Occidental } \\
\text { Rd., Amity } \\
\text { Creek Park, } \\
\text { \# } 3\end{array}$ & $46^{\circ} 51^{\prime} 25^{\prime \prime}$ & $92^{\circ} 00^{\prime} 48^{\prime \prime}$ & ns & no & ns & ns \\
\hline 15 & $\begin{array}{l}\text { Amity Creek at } \\
\text { Occidental } \\
\text { Rd., Amity } \\
\text { Creek Park, } \\
\text { \# } 4\end{array}$ & $46^{\circ} 51^{\prime 2} 29^{\prime \prime}$ & $92^{\circ} 00^{\prime} 47^{\prime \prime}$ & ns & no & ns & ns \\
\hline 16 & $\begin{array}{l}\text { Amity Creek at } \\
\text { Occidental } \\
\text { Rd., Amity } \\
\text { Creek Park, } \\
\text { \# } 5\end{array}$ & $46^{\circ} 51^{\prime} 35^{\prime \prime}$ & $92^{\circ} 00^{\prime} 51^{\prime \prime}$ & ns & no & Bedrock controlled channel. & ns \\
\hline 17 & $\begin{array}{l}\text { Amity Creek at } \\
\text { Occidental } \\
\text { Rd., Amity } \\
\text { Creek Park, } \\
\text { \# } 6\end{array}$ & $46^{\circ} 51^{\prime} 39^{\prime \prime}$ & $92^{\circ} 00^{\prime} 53^{\prime \prime}$ & ns & no & Bedrock controlled channel. & $\begin{array}{l}\text { Pools caused by } \\
\text { scour at base of } \\
\text { falls. }\end{array}$ \\
\hline 18 & $\begin{array}{l}\text { Amity Creek at } \\
\text { Skyline Rd. }\end{array}$ & $46^{\circ} 51^{\prime} 40^{\prime \prime}$ & $92^{\circ} 00^{\prime} 59^{\prime \prime}$ & ns & no & Bedrock controlled channel. & ns \\
\hline 19 & $\begin{array}{l}\text { Lester River at } \\
\text { Superior St. }\end{array}$ & $46^{\circ} 50^{\prime} 21^{\prime \prime}$ & $92^{\circ} 00^{\prime} 25^{\prime \prime}$ & $\mathrm{ns}$ & no & Bedrock controlled channel. & $\begin{array}{l}\text { Pools caused by } \\
\text { scour at base of } \\
\text { falls. }\end{array}$ \\
\hline 20 & $\begin{array}{l}\text { Lester River at } \\
\text { Strand Rd. }\end{array}$ & $46^{\circ} 53^{\prime} 16^{\prime \prime}$ & $91^{\circ} 59^{\prime} 29^{\prime \prime}$ & $\mathrm{ns}$ & no & $\begin{array}{l}\text { Channel appears entrenched but also has some } \\
\text { depositional features. }\end{array}$ & ns \\
\hline 21 & $\begin{array}{l}\text { Lester River } \\
\text { Tributary at } \\
\text { Strand Rd. }\end{array}$ & $46^{\circ} 53^{\prime} 16^{\prime \prime}$ & $91^{\circ} 59^{\prime} 42^{\prime \prime}$ & $\mathrm{ns}$ & no & ns & ns \\
\hline 22 & $\begin{array}{l}\text { Lester River } \\
\text { Tributary at } \\
\text { Beyer Rd. }\end{array}$ & $46^{\circ} 53^{\prime} 41^{\prime \prime}$ & $92^{\circ} 00^{\prime} 21^{\prime \prime}$ & ns & no & Ditch-like, transitional/depositional? & ns \\
\hline 23 & $\begin{array}{l}\text { Lester River at } \\
\text { North Tischer } \\
\text { Rd. }\end{array}$ & $46^{\circ} 54^{\prime} 27^{\prime \prime}$ & $92^{\circ} 00^{\prime} 20^{\prime \prime}$ & 28.51 & yes & $\begin{array}{l}\text { Bluffs/valley sides are currently stable, } \\
\text { widening a problem, accum. of fines in pools, } \\
\text { unstable banks but some toe protection from } \\
\text { co/bo in terrace deposits (glacial meltwater } \\
\text { related); pre-settlement tree trunks exposed } \\
\text { along banks. Sediment accumulation around } \\
\text { fallen trees. }\end{array}$ & $\begin{array}{l}\text { Fine sediment } \\
\text { accumulation. }\end{array}$ \\
\hline 24 & $\begin{array}{l}\text { Lester River } \\
\text { at Hwy 43, } \\
\text { Lismore Rd. }\end{array}$ & $46^{\circ} 56^{\prime} 18^{\prime \prime}$ & $92^{\circ} 04^{\prime} 26^{\prime \prime}$ & ns & no & Stable grassy banks, base flow driven flow. & $\begin{array}{l}\text { Pool on ds side } \\
\text { culvert. }\end{array}$ \\
\hline 25 & $\begin{array}{l}\text { Lester River at } \\
\text { Arnold Rd., } \\
\text { \#1 }\end{array}$ & $46^{\circ} 58^{\prime} 40^{\prime \prime}$ & $92^{\circ} 05^{\prime} 26^{\prime \prime}$ & 2.94 & no & $\begin{array}{l}\text { Depositional, organic debris accumulation } \\
\text { during low flow, but scour down to clay during } \\
\text { floods. }\end{array}$ & $\begin{array}{l}\text { Scour pool at tribu- } \\
\text { tary confluence. }\end{array}$ \\
\hline
\end{tabular}




\begin{tabular}{lcccc}
\hline LWD notes & Overbank & $\begin{array}{c}\text { Estimate of } \\
\text { streamflow } \\
\text { (cfs) }\end{array}$ & Sensitivity to change & Additional notes \\
\hline $\mathrm{ns}$ & $\mathrm{ns}$ & $\mathrm{ns}$ & $\mathrm{ns}$ & $\begin{array}{l}\text { Local erosion, potential for } \\
\text { landslides. }\end{array}$ \\
& & & &
\end{tabular}

$\begin{array}{lllll}\mathrm{ns} & \mathrm{ns} & \mathrm{ns} & \text { none } \\ & & & & \\ & & & & \\ \mathrm{ns} & \mathrm{ns} & \mathrm{ns} & \mathrm{ns} & \text { none }\end{array}$

\begin{tabular}{|c|c|c|c|c|}
\hline ns & ns & ns & ns & none \\
\hline LWD rare. & None, outcrop. & ns & $\begin{array}{l}\text { Local human activity—rearranging } \\
\text { rocks to deepen pools. }\end{array}$ & $\begin{array}{l}\text { Park/trails along both sides, } \\
\text { local erosion from trail runoff, } \\
\text { potential landslides. }\end{array}$ \\
\hline LWD occas. & ns & ns & Bank erosion, bluff erosion. & none \\
\hline ns & ns & ns & ns & Alder thicket. \\
\hline ns & ns & $<.25$ & ns & Alder thicket. \\
\hline $\begin{array}{l}\text { Us source for LWD. Large } \\
\text { log jam ds of bridge and } \\
\text { above sampled reach-some } \\
\text { of it hanging above chan- } \\
\text { nel. Source for LWD in } \\
\text { reach from trees falling in } \\
\text { from banks from widening. } \\
\text { Appears that most LWD is } \\
\text { transported ds during floods. }\end{array}$ & $\begin{array}{l}\text { Abandoned channel at or } \\
\text { slightly lower in elevation } \\
\text { than modern channel. } \\
\text { Abandoned channel } \\
\text { filled with wood and } 4 \mathrm{ft} \\
\text { loam/fines. Buried soil at } \\
\text { about } 3 \mathrm{ft} \text { below surface. } \\
\text { On FP, } 1 \mathrm{ft} \text { sand/loam } \\
\text { over gravel. }\end{array}$ & $4-5$ & $\begin{array}{l}\text { High-potential to migrate or widen } \\
\text { into valley sides and increase erosion/ } \\
\text { sediment input. }\end{array}$ & $\begin{array}{l}\text { Bridge is not blocking fish } \\
\text { passage. }\end{array}$ \\
\hline None apparent. & ns & $3-4$ & ns & $\begin{array}{l}\text { Culvert does not block fish } \\
\text { passage. }\end{array}$ \\
\hline $\begin{array}{l}\text { LWD from snags on banks, } \\
\text { some sediment trapping } \\
\text { function. }\end{array}$ & $\begin{array}{l}2 \mathrm{ft} \text { organic muck over } \\
1 \mathrm{ft} \text { peat over glacial lake } \\
\text { clay. }\end{array}$ & 0 & $\begin{array}{l}\text { Us changes in runoff promotes channel } \\
\text { scour. Deposition of organic debris } \\
\text { during low flow. No source observed } \\
\text { for gravel. }\end{array}$ & $\begin{array}{l}\text { Old culvert does not block fish } \\
\text { passage. }\end{array}$ \\
\hline
\end{tabular}


Table 7. Reach characteristics at rapid assessment and intensive survey field sites for Duluth-area streams, Minn., 2003-2004 -Continued.

[USGS, U.S. Geological Survey; mi, mile; ft, foot; LWD, large woody debris; cfs, cubic foot per second; ${ }^{\circ}$, degree; '. minute; ", second; mi², square mile; $\%$, percent; gv, gravel; co, cobble; FP, flood plain; m, medium; c, coarse; ns, not sampled; bf, bankfull; w, width; d, depth; bo, boulder; ds, downstream; us, upstream; , approximately; sed, sediment; <, less than; occas., occasional; ns, not surveyed; accum, accumulation; trib, tributary]

\begin{tabular}{|c|c|c|c|c|c|c|c|}
\hline $\begin{array}{l}\text { USGS } \\
\text { field } \\
\text { number }\end{array}$ & $\begin{array}{l}\text { Stream } \\
\text { name }\end{array}$ & Latitude & Longitude & $\begin{array}{l}\text { Drainage } \\
\text { area } \\
\left(\mathrm{mi}^{2}\right)\end{array}$ & $\begin{array}{l}\text { Measured } \\
\text { reach } \\
\text { slope }(\%)\end{array}$ & Channel geomorphic setting & Pool notes \\
\hline 26 & $\begin{array}{l}\text { Lester River at } \\
\text { Arnold Rd. } \\
\text { \#2 }\end{array}$ & $46^{\circ} 56^{\prime} 54^{\prime \prime}$ & $92^{\circ} 05^{\prime} 26^{\prime \prime}$ & ns & no & Depositional? Natural levees of muck. & $\begin{array}{l}\text { Pooled at cement } \\
\text { box culvert. }\end{array}$ \\
\hline 27 & $\begin{array}{l}\text { Lester River at } \\
\text { Emerson Rd. }\end{array}$ & $46^{\circ} 57^{\prime} 10^{\prime \prime}$ & $92^{\circ} 05^{\prime} 44^{\prime \prime}$ & ns & no & Same as site 25 and 26. & $\begin{array}{l}\text { Scour pool ds of } \\
\text { road culvert. }\end{array}$ \\
\hline 28 & $\begin{array}{l}\text { Lester River at } \\
\text { Howard Gne- } \\
\text { sen Rd., \#1 }\end{array}$ & $46^{\circ} 57^{\prime} 45^{\prime \prime}$ & $92^{\circ} 06^{\prime} 42^{\prime \prime}$ & ns & no & $\begin{array}{l}\text { Lowland setting, more riparian woody vegeta- } \\
\text { tion than sites } 25-27 \text {, organic deposits in chan- } \\
\text { nel bottom, evidence of minor downcutting. }\end{array}$ & ns \\
\hline 29 & $\begin{array}{l}\text { Lester River at } \\
\text { Howard Gne- } \\
\text { sen Rd., \#2 }\end{array}$ & $46^{\circ} 58^{\prime} 36^{\prime \prime}$ & $92^{\circ} 06^{\prime} 43^{\prime \prime}$ & $\mathrm{ns}$ & no & Similar to site 28 , maybe a little less erosion. & ns \\
\hline 30 & $\begin{array}{l}\text { Lester River at } \\
\text { Hwy 37, Jean } \\
\text { Duluth Rd. }\end{array}$ & $46^{\circ} 55^{\prime} 18^{\prime \prime}$ & $92^{\circ} 02^{\prime} 54^{\prime \prime}$ & 19.28 & yes & $\begin{array}{l}\text { Occasional landslides, widening, no evidence } \\
\text { of incision, not as unstable as site } 23 \text {, undercut } \\
\text { banks. }\end{array}$ & $\begin{array}{l}\text { Formed by riffles, } \\
\text { enhanced by LWD. }\end{array}$ \\
\hline 31 & $\begin{array}{l}\text { Lester River near } \\
\text { Lester River } \\
\text { Rd. }\end{array}$ & $46^{\circ} 50^{\prime} 32^{\prime \prime}$ & $92^{\circ} 00^{\prime} 21 "$ & 36.45 & yes & $\begin{array}{l}\text { Bedrock controlled channel, rocks scoured, in- } \\
\text { creases in bankfull width/depth, fine sediment } \\
\text { accum. in pools, channel widening with local } \\
\text { increases in valley width (bedrock controlled). }\end{array}$ & $\begin{array}{l}\text { Pools caused by } \\
\text { scour at base of } \\
\text { falls, levels raised } \\
\text { by humans. }\end{array}$ \\
\hline 32 & $\begin{array}{l}\text { Miller Creek at } \\
\text { Ridgeview Rd. }\end{array}$ & $46^{\circ} 51^{\prime} 04^{\prime \prime}$ & $92^{\circ} 09^{\prime} 45^{\prime \prime}$ & $\mathrm{ns}$ & no & ns & ns \\
\hline 33 & $\begin{array}{l}\text { Miller Creek at } \\
\text { Swan Lake } \\
\text { Rd. }\end{array}$ & $46^{\circ} 49^{\prime} 43^{\prime \prime}$ & $92^{\circ} 10^{\prime} 34^{\prime \prime}$ & $\mathrm{ns}$ & no & $\begin{array}{l}\text { Evidence of incision and widening, organic } \\
\text { debris deposition common (peat eroded from } \\
\text { us reaches). }\end{array}$ & $\begin{array}{l}\text { Silt accumulation } \\
\text { in scour hole ds of } \\
\text { culvert. }\end{array}$ \\
\hline 34 & $\begin{array}{l}\text { Miller Creek } \\
\text { upstream of } \\
\text { Chambers- } \\
\text { berg Ave. }\end{array}$ & $46^{\circ} 47^{\prime} 46^{\prime \prime}$ & $92^{\circ} 09^{\prime} 37^{\prime \prime}$ & 7.44 & yes & $\begin{array}{l}\text { Massive erosion problem, new scour channels, } \\
\text { widening, incision due to large increases in } \\
\text { runoff volume from shopping area. }\end{array}$ & $\begin{array}{l}\text { Pools from } \\
\text { riffle/pool complex, } \\
\text { LWD, and boulder. } \\
\text { Siltation in large } \\
\text { pool between } \\
\text { bottom of reach } \\
\text { and driveway } \\
\text { crossing. }\end{array}$ \\
\hline 35 & $\begin{array}{l}\text { Miller Creek at } \\
\text { Anderson Rd. }\end{array}$ & $46^{\circ} 47^{\prime} 36^{\prime \prime}$ & $92^{\circ} 09^{\prime} 26^{\prime \prime}$ & $\mathrm{ns}$ & yes & $\begin{array}{l}\text { Signs of widening, more so downstream of } \\
\text { culvert, upstream banks reinforced with riprap, } \\
\text { no indications of siltation. }\end{array}$ & 1 step pool. \\
\hline 36 & $\begin{array}{l}\text { Miller Creek be- } \\
\text { tween 2nd and } \\
\text { 3rd St. and } \\
\text { 26th Ave. }\end{array}$ & $46^{\circ} 45^{\prime} 51^{\prime \prime}$ & $92^{\circ} 07^{\prime} 58^{\prime \prime}$ & $\mathrm{ns}$ & no & $\begin{array}{l}\text { Stable, wide short cascade between under- } \\
\text { ground segments. }\end{array}$ & none \\
\hline 37 & $\begin{array}{l}\text { Miller Creek at } \\
\text { 10th St. }\end{array}$ & $46^{\circ} 46^{\prime} 15^{\prime \prime}$ & $92^{\circ} 08^{\prime} 23^{\prime \prime}$ & $\mathrm{ns}$ & no & $\begin{array}{l}\text { Somewhat stable because of bedrock, increase } \\
\text { in side scour because of increase in flood size, } \\
\text { also some indication of incision under bridge } \\
\text { (1927), maybe a bit of plucking going on. }\end{array}$ & $\begin{array}{l}\text { Pools at bottom of } \\
\text { falls. }\end{array}$ \\
\hline
\end{tabular}




\begin{tabular}{|c|c|c|c|c|}
\hline LWD notes & $\begin{array}{c}\text { Overbank } \\
\text { sedimentation }\end{array}$ & $\begin{array}{l}\text { Estimate of } \\
\text { streamflow } \\
\quad \text { (cfs) }\end{array}$ & Sensitivity to change & Additional notes \\
\hline $\begin{array}{l}\text { LWD from snags on banks, } \\
\text { water-table changes related to } \\
\text { road construction. }\end{array}$ & ns & 0 & Similar to site 25 . & $\begin{array}{l}\text { Cement box culvert does not } \\
\text { block fish passage. }\end{array}$ \\
\hline $\begin{array}{l}\text { LWD from snags on banks, } \\
\text { water-table changes related to } \\
\text { road construction. }\end{array}$ & Appears similar to site 25 . & $<0.25$ & Similar to site 25 . & No notes on culvert condition. \\
\hline ns & ns & 1.5 & Similar to sites $25-27$. & $\begin{array}{l}\text { Culvert does not block fish } \\
\text { passage. }\end{array}$ \\
\hline ns & ns & ns & Similar to site $25-28$. & No notes on culvert condition. \\
\hline $\begin{array}{l}\text { Mainly from bank widening, } \\
\text { occas. carried in from us. }\end{array}$ & $\begin{array}{l}\text { On FP, } 1 \mathrm{ft} \text { sand over } 0.45 \\
\mathrm{ft} \text { organic-rich sand with } \\
\text { common wood/organic } \\
\text { debris over co/gv. Com- } \\
\text { mon woody debris at } 1 \mathrm{ft} \\
\text { below FP surface, related } \\
\text { probably to European } \\
\text { settlement. }\end{array}$ & 4 & $\begin{array}{l}\text { Terrace and valley side cuts source of } \\
\text { sediment to ds reaches, sensitive to us } \\
\text { sources of sediment, also additional } \\
\text { runoff from us may cause widening/ } \\
\text { meandering and more terrace/ valley } \\
\text { side cuts, no evidence of incision. }\end{array}$ & $\begin{array}{l}\text { Half culvert in not blocking fish } \\
\text { passage. No signs of erosion. } \\
\text { Painted rocks in stream. }\end{array}$ \\
\hline Lack of LWD. & None, outcrop. & ns & $\begin{array}{l}\text { Gullying from tributaries intersecting } \\
\text { valley sides, landslides from trail runoff, } \\
\text { us inputs from flood/scour, sediment } \\
\text { flushed through reach, occasional gravel } \\
\text { bars. }\end{array}$ & $\begin{array}{l}\text { Thick algae on rocks, heavily } \\
\text { used trails. }\end{array}$ \\
\hline ns & ns & ns & Potential effects from us landfill. & none \\
\hline $\begin{array}{l}\text { Abundant LWD from banks, } \\
\text { helps to stabilize banks. }\end{array}$ & ns & $\sim 1$ & Us inputs of sediment and runoff. & $\begin{array}{l}\text { Oil slick on surface, water odor, } \\
\text { receives runoff from airport } \\
\text { parking lot, abundant algae, cul- } \\
\text { vert does not block fish passage. }\end{array}$ \\
\hline $\begin{array}{l}\text { Abundant, coming from } \\
\text { banks, causing local scour/ } \\
\text { bank erosion, probably source } \\
\text { of LWD to downstream } \\
\text { reaches. }\end{array}$ & $\begin{array}{l}\text { Center of island between } \\
\text { channels: } 1-1.5 \mathrm{ft} \text { sandy } \\
\text { loam over gravel. Buried } \\
\text { logs, wood common at } \\
1.5 \mathrm{ft} \text {, observed eroding } \\
\text { from bank toe as well. }\end{array}$ & $\sim 4$ & $\begin{array}{l}\text { High—runoff, sediment source for } \\
\text { downstream reaches. }\end{array}$ & $\begin{array}{l}\text { Bridge does not block fish } \\
\text { passage; landowner said no fish } \\
\text { spawning in } 2003 \text { because of } \\
\text { fish kill last winter and dry sum- } \\
\text { mer. Landowner thought stream } \\
\text { change happened after com- } \\
\text { mercial development on north } \\
\text { side of highway. Some springs } \\
\text { downstream of bridge. }\end{array}$ \\
\hline $\begin{array}{l}\text { Occas. LWD from alder along } \\
\text { banks, some sediment trap- } \\
\text { ping, some bank stabilization. }\end{array}$ & ns, residential backyards. & $\sim 4$ & $\begin{array}{l}\text { High-runoff, sediment source for } \\
\text { downstream reaches, human alterations } \\
\text { to control channel changes. }\end{array}$ & $\begin{array}{l}\text { Culvert does not block fish pas- } \\
\text { sage, erosion around upstream } \\
\text { side of culvert. }\end{array}$ \\
\hline Minimal. & ns & ns & Highly altered and reinforced. & $\begin{array}{l}\text { No fish passage through culvert, } \\
\text { short open section between } \\
\text { underground segments. }\end{array}$ \\
\hline $\begin{array}{l}\text { Occas LWD from valley } \\
\text { sides, transported out during } \\
\text { floods. }\end{array}$ & None, outcrop. & $4 ?$ & $\begin{array}{l}\text { Valley side failures from road/trail run- } \\
\text { off, and entrance of storm sewers/tribs. } \\
\text { road washouts common. }\end{array}$ & none \\
\hline
\end{tabular}


Table 7. Reach characteristics at rapid assessment and intensive survey field sites for Duluth-area streams, Minn., 2003-2004 -Continued.

[USGS, U.S. Geological Survey; mi, mile; ft, foot; LWD, large woody debris; cfs, cubic foot per second; ${ }^{\circ}$, degree; '. minute; ", second; mi², square mile; $\%$, percent; gv, gravel; co, cobble; FP, flood plain; m, medium; c, coarse; ns, not sampled; bf, bankfull; w, width; d, depth; bo, boulder; ds, downstream; us, upstream; , approximately; sed, sediment; <, less than; occas., occasional; ns, not surveyed; accum, accumulation; trib, tributary]

\begin{tabular}{|c|c|c|c|c|c|c|c|}
\hline $\begin{array}{l}\text { USGS } \\
\text { field } \\
\text { number }\end{array}$ & $\begin{array}{l}\text { Stream } \\
\text { name }\end{array}$ & Latitude & Longitude & $\begin{array}{l}\text { Drainage } \\
\text { area } \\
\left(\mathrm{mi}^{2}\right)\end{array}$ & $\begin{array}{l}\text { Measured } \\
\text { reach } \\
\text { slope }(\%)\end{array}$ & Channel geomorphic setting & Pool notes \\
\hline 38 & $\begin{array}{l}\text { Mission Creek at } \\
\text { Hwy } 23\end{array}$ & $46^{\circ} 39^{\prime} 39^{\prime \prime}$ & $92^{\circ} 16^{\prime} 32^{\prime \prime}$ & $\mathrm{ns}$ & no & $\begin{array}{l}\text { Maybe some widening, no signs of incision, } \\
\text { man made channel oversized. }\end{array}$ & Rare or absent. \\
\hline 39 & $\begin{array}{l}\text { Mission Creek } \\
\text { at 131st Ave. } \\
\text { West }\end{array}$ & $46^{\circ} 40^{\prime} 13^{\prime \prime}$ & $92^{\circ} 16^{\prime} 37^{\prime \prime}$ & $\mathrm{ns}$ & no & $\begin{array}{l}\text { Impoundment collected about } 1-2 \text { feet gravel } \\
\text { disturbed by dam. }\end{array}$ & $\mathrm{ns}$ \\
\hline 40 & $\begin{array}{l}\text { Mission Creek at } \\
\text { Helburg Rd. }\end{array}$ & $46^{\circ} 42^{\prime} 11^{\prime \prime}$ & $92^{\circ} 20^{\prime} 02^{\prime \prime}$ & $\mathrm{ns}$ & no & $\mathrm{ns}$ & None present. \\
\hline 41 & $\begin{array}{l}\text { Kingsbury Creek } \\
\text { south of Alice } \\
\text { St and 1st } \\
\text { Ave. North }\end{array}$ & $46^{\circ} 44^{\prime} 13^{\prime \prime}$ & $92^{\circ} 13^{\prime} 12^{\prime \prime}$ & $\mathrm{ns}$ & no & $\begin{array}{l}\text { Widening - alders falling, exposed roots } \\
\text { common, grassy banks are undercut, fine sed } \\
\text { deposition between boulders and in pools. }\end{array}$ & $\begin{array}{l}\text { Pools related to } \\
\text { step/pool channel } \\
\text { units. }\end{array}$ \\
\hline 42 & $\begin{array}{c}\text { Keene Creek at } \\
\text { Bristol St. }\end{array}$ & $46^{\circ} 44^{\prime} 15^{\prime \prime}$ & $92^{\circ} 10^{\prime} 35^{\prime \prime}$ & $\mathrm{ns}$ & no & $\begin{array}{l}\text { Stable, artificial hardening from willows and } \\
\text { boulder riprap on banks, drop structure at end } \\
\text { of park. }\end{array}$ & $\begin{array}{l}\text { None except for } \\
\text { at bottom of drop } \\
\text { structure. }\end{array}$ \\
\hline 43 & $\begin{array}{l}\text { Chester Creek at } \\
\text { Triggs Rd. }\end{array}$ & $46^{\circ} 49^{\prime} 12^{\prime \prime}$ & $92^{\circ} 06^{\prime} 40^{\prime \prime}$ & 3.00 & yes & $\begin{array}{l}\text { Unstable, multiple channels, similar to site } 34 \\
\text { except maybe a little more incised. Degrading } \\
\text { and widening, flood channels common. }\end{array}$ & $\begin{array}{l}\text { Pools related to } \\
\text { riffle/pool sequenc- } \\
\text { es and LWD. }\end{array}$ \\
\hline
\end{tabular}

\begin{tabular}{|c|c|c|c|c|c|c|c|}
\hline 44 & $\begin{array}{l}\text { Miller Creek at } \\
\text { Lake Superior } \\
\text { College }\end{array}$ & $46^{\circ} 47^{\prime} 06^{\prime \prime}$ & $92^{\circ} 08^{\prime} 52^{\prime \prime}$ & 8.26 & yes & $\begin{array}{l}\text { Signs of widening in areas without bank } \\
\text { protection and some slight incision. }\end{array}$ & No pools in reach. \\
\hline 45 & $\begin{array}{l}\text { U.S. Steel Creek } \\
\text { upstream of } \\
\text { Hwy. } 23\end{array}$ & $46^{\circ} 40^{\prime} 30^{\prime \prime}$ & $92^{\circ} 13^{\prime} 15^{\prime \prime}$ & ns & no & Extensive bank erosion along railroad track. & ns \\
\hline 46 & $\begin{array}{c}\text { Coffee Creek at } \\
\text { North } 19 \text { 1/2 } \\
\text { Ave. West }\end{array}$ & $46^{\circ} 46^{\prime} 20^{\prime \prime}$ & $92^{\circ} 08^{\prime} 04^{\prime \prime}$ & ns & no & Bedrock channel, possible enlargement. & $\mathrm{ns}$ \\
\hline 47 & $\begin{array}{l}\text { Merritt Creek } \\
\text { tributary at } \\
\text { Skyline Drive }\end{array}$ & $46^{\circ} 45^{\prime} 45^{\prime \prime}$ & $92^{\circ} 09^{\prime} 38^{\prime \prime}$ & ns & no & Bedrock channel, possible widening. & $\begin{array}{l}\text { Step pools- } \\
\text { garbage, rare. }\end{array}$ \\
\hline 48 & $\begin{array}{l}\text { Merritt Creek at } \\
\text { Skyline Drive }\end{array}$ & $46^{\circ} 45^{\prime} 35^{\prime \prime}$ & $92^{\circ} 09^{\prime} 54^{\prime \prime}$ & ns & no & Bedrock channel, possible enlargement. & ns \\
\hline
\end{tabular}




\begin{tabular}{|c|c|c|c|c|}
\hline LWD notes & $\begin{array}{c}\text { Overbank } \\
\text { sedimentation }\end{array}$ & $\begin{array}{l}\text { Estimate of } \\
\text { streamflow } \\
\text { (cfs) }\end{array}$ & Sensitivity to change & Additional notes \\
\hline $\begin{array}{l}\text { Rare LWD, from bank, no } \\
\text { function. }\end{array}$ & None? & $3 ?$ & $\begin{array}{l}\text { Low—human alterations, reinforce- } \\
\text { ments. }\end{array}$ & $\begin{array}{l}\text { Bridge area looks stable, no } \\
\text { signs of erosion, does not block } \\
\text { fish passage. }\end{array}$ \\
\hline ns & ns & ns & Unknown. & $\begin{array}{l}\text { Dam has been out for some } \\
\text { time, } 1997 \text { air photo shows } \\
\text { disturbance at site as well. Not } \\
\text { very good location. }\end{array}$ \\
\hline LWD from bank. & ns & 0 & Runoff from interstate, road crossings. & $\begin{array}{l}\text { Road crossing interfering with } \\
\text { drainage. }\end{array}$ \\
\hline $\begin{array}{l}\text { LWD from bank, alders fall- } \\
\text { ing in, some hanging above } \\
\text { channel ds, no function. }\end{array}$ & ns & $2 ?$ & $\begin{array}{l}\text { High—if runoff increases will widen } \\
\text { more. }\end{array}$ & none \\
\hline $\begin{array}{l}\text { LWD rare, maybe from } \\
\text { upstream, no function. }\end{array}$ & ns & $2 ?$ & $\begin{array}{l}\text { Low_human alterations, reinforce- } \\
\text { ments. }\end{array}$ & $\begin{array}{l}3 \text { foot drop in drop structure is } \\
\text { fish barrier? }\end{array}$ \\
\hline $\begin{array}{l}\text { LWD common from banks } \\
\text { and upstream, provides } \\
\text { multiple functions. }\end{array}$ & $\begin{array}{l}0.5-1.5 \mathrm{ft} \text { sand loam over } \\
\mathrm{gv} / \mathrm{co} .\end{array}$ & $<1 / 4$ & $\begin{array}{l}\text { High—widening/downcutting—pro- } \\
\text { vides sediment to ds reaches. }\end{array}$ & $\begin{array}{l}\text { Bedrock geologic map (1949) } \\
\text { shows gridwork of streets in } \\
\text { the section, not there today. Old } \\
\text { building foundation on right } \\
\text { bank of cross section } 2 \text {, maybe } \\
\text { more houses there } 50 \text { years ago } \\
\text { than now? }\end{array}$ \\
\hline $\begin{array}{l}\text { LWD mainly from banks/ } \\
\text { valley side slope. }\end{array}$ & $\begin{array}{l}\text { Minimal on right side, } \\
1.5 \mathrm{ft} \text { sand } \mathrm{w} / \text { boulder. } \\
\text { No left FP; all riprap on } \\
\text { left side. }\end{array}$ & $4 ?$ & $\begin{array}{l}\text { Slight to moderate-hardened banks } \\
\text { and bottom, subject to side scour from } \\
\text { increases in flood size. }\end{array}$ & $\begin{array}{l}\text { Artificial hardening of left side } \\
\text { where storm sewer parallels } \\
\text { creek for long distance, prob- } \\
\text { ably down to STP at lake. }\end{array}$ \\
\hline ns & ns & ns & Subject to widening. & $\begin{array}{l}\text { Recent rebuilding of banks, no } \\
\text { veg, silt fence failing. }\end{array}$ \\
\hline ns & none & $\mathrm{ns}$ & Subject to enlargement. & Disturbed land cover. \\
\hline Rare, us side of bridge. & none & $2 ?$ & $\begin{array}{l}\text { Subject to widening, urban development } \\
\text { upstream. }\end{array}$ & $\begin{array}{l}\text { Trails along stream, new } \\
\text { residential development us, } \\
\text { wetlands common us. }\end{array}$ \\
\hline $\mathrm{ns}$ & none & ns & $\begin{array}{l}\text { Subject to enlargement, urban develop- } \\
\text { ment upstream. }\end{array}$ & $\begin{array}{l}\text { Cannot access from roads, no } \\
\text { parking. }\end{array}$ \\
\hline
\end{tabular}




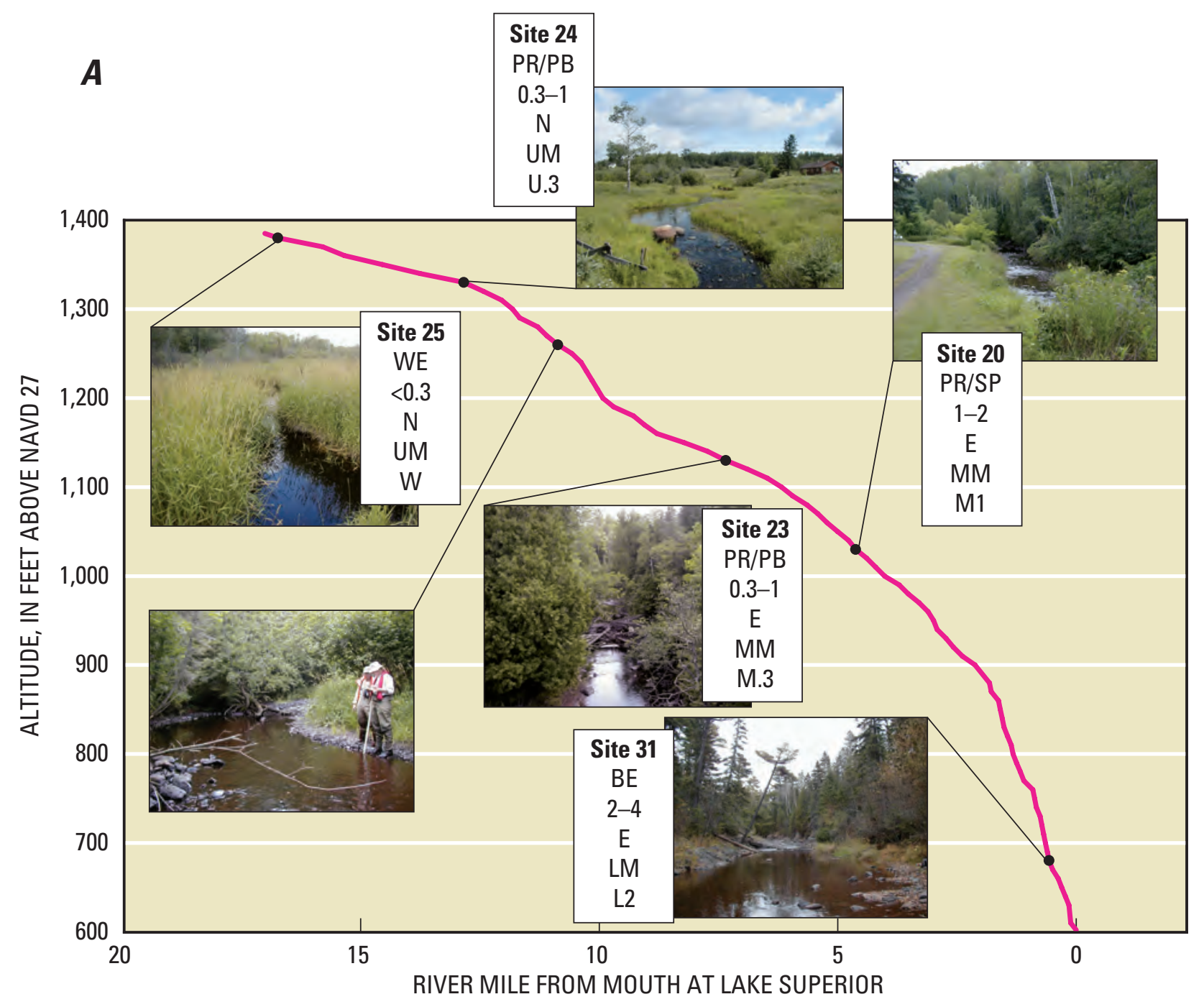

\section{EXPLANATION}

\begin{tabular}{|c|c|}
\hline Site 30 & ........ U.S. Geological Survey site number (fig. 1) \\
\hline PR & Channel type \\
\hline $1-2$ & Slope category, in percent \\
\hline $\mathrm{E}$ & Valley type \\
\hline MM & Drainage network position \\
\hline M1 … & Geomorphic Segment Category (GSC) \\
\hline
\end{tabular}

Figure 11. Longitudinal profiles for $\boldsymbol{A}$, Lester River and $\boldsymbol{B}$, Miller Creek, in the Duluth, Minn., area. See table 6 for explanation of abbreviations. $(<$, less than) 


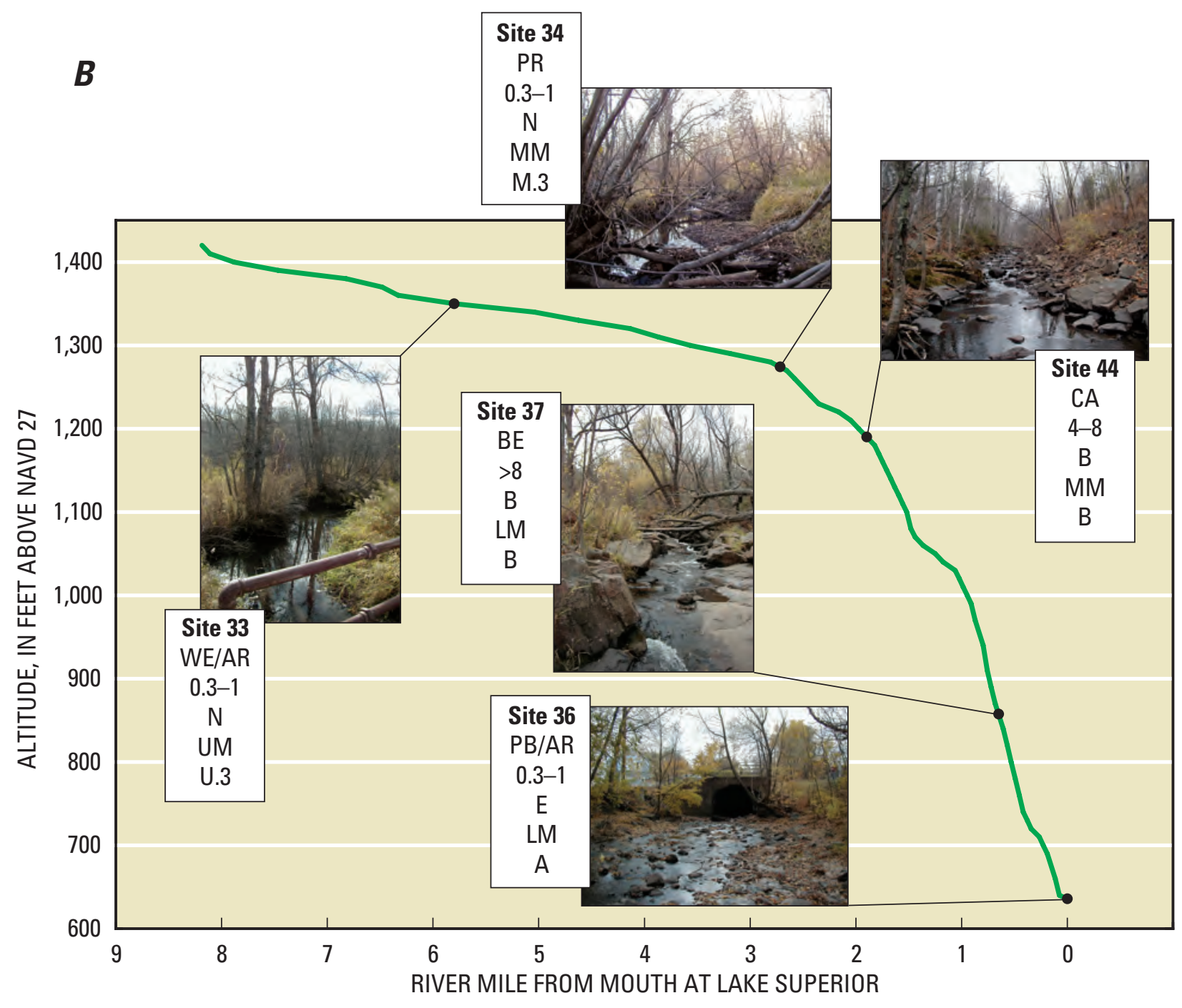

EXPLANATION

\begin{tabular}{|c|c|}
\hline Site 30 & U.S. Geological Survey site number (fig. \\
\hline PR & Channel type \\
\hline $1-2$ & Slope category, in percent \\
\hline $\mathrm{E}$ & Valley type \\
\hline MM & Drainage network position \\
\hline M1 - & Geomorphic Segment Category (GSC) \\
\hline
\end{tabular}

Figure 11. Longitudinal profiles for $\boldsymbol{A}$, Lester River and $\boldsymbol{B}$, Miller Creek, in the Duluth, Minn., area. See table 6 for explanation of abbreviations-Continued. (<, less than) 
Table 8. Potential channel types in each slope/valley category for Duluth-area streams, Minn.

$[<$, less than; >, greater than; channel types: WE, wetland; PR, pool-riffle; PB, plane-bed; SP, step-pool; BE, bedrock; CA, cascade; CO, colluvial; na, not applicable]

\begin{tabular}{cccccc}
\hline $\begin{array}{c}\text { Slope category } \\
\text { (percent) }\end{array}$ & No valley & Confined & Entrenched & Alluvial & Bedrock \\
\hline$<0.3$ & $\mathrm{WE}$ & $\mathrm{na}$ & $\mathrm{na}$ & $\mathrm{na}$ & $\mathrm{na}$ \\
$0.3-1$ & $\mathrm{WE} / \mathrm{PR}$ & $\mathrm{na}$ & $\mathrm{na}$ & $\mathrm{PB} / \mathrm{PR}$ & $\mathrm{na}$ \\
$>1-2$ & $\mathrm{PR}$ & $\mathrm{PB}$ & $\mathrm{PB}$ & $\mathrm{na}$ & $\mathrm{PB} / \mathrm{SP} / \mathrm{BE}$ \\
$>2-4$ & $\mathrm{~PB}$ & $\mathrm{SP} / \mathrm{CA} / \mathrm{CO}$ & $\mathrm{SP} / \mathrm{CA} / \mathrm{BE} / \mathrm{CO}$ & $\mathrm{na}$ & $\mathrm{SP} / \mathrm{CA} / \mathrm{BE} / \mathrm{CO}$ \\
$>4-8$ & $\mathrm{na}$ & $\mathrm{CA} / \mathrm{BE} / \mathrm{CO}$ & $\mathrm{CA} / \mathrm{BE} / \mathrm{CO}$ & $\mathrm{na}$ & $\mathrm{BE} / \mathrm{CA} / \mathrm{CO}$ \\
$>8$ & $\mathrm{na}$ & $\mathrm{BE}$ & $\mathrm{BE}$ & $\mathrm{na}$ & $\mathrm{BE}$ \\
\hline
\end{tabular}

$\boldsymbol{A}$

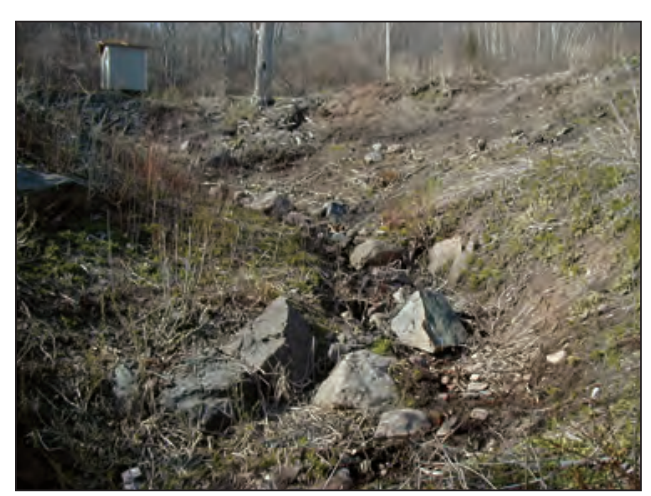

Coffee Creek

Site 46

\section{B}

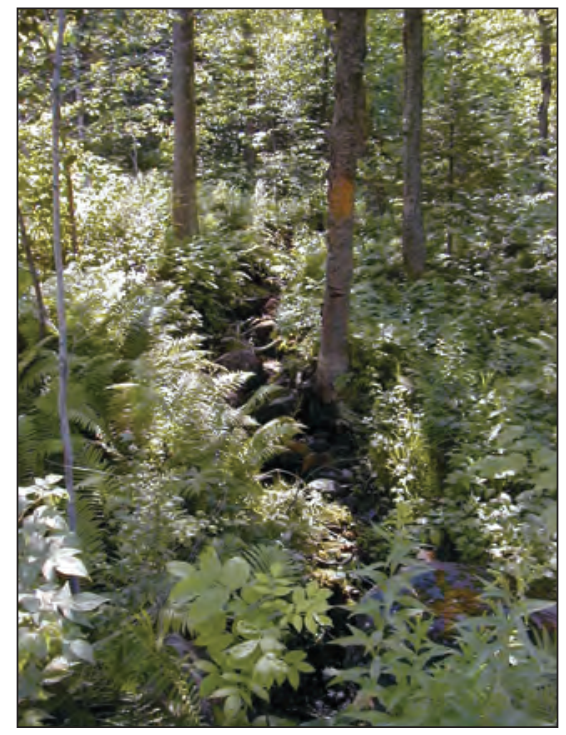

Stewart Creek tributary

Site 2
C

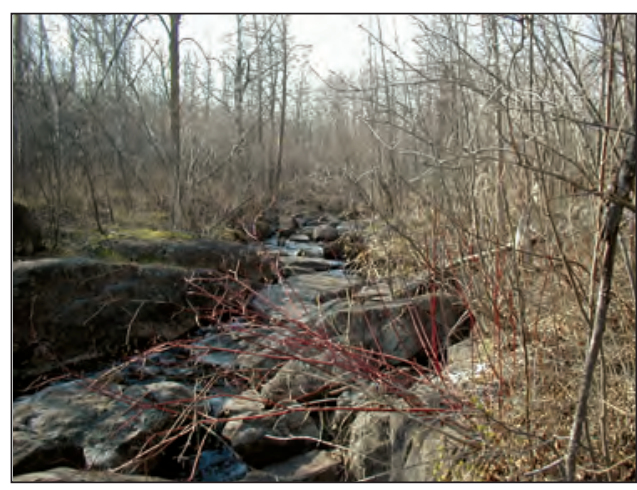

Merritt Creek tributary

Site 47

D

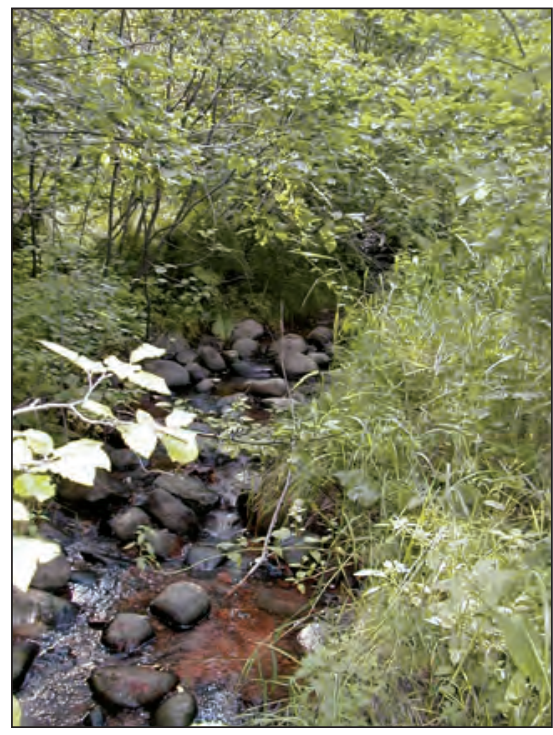

Keene Creek tributary

Site 5

Figure 12. Photographs of rapid assessment sites on tributaries affected by bedrock: $\boldsymbol{A}$, site 46 on Coffee Creek, $\boldsymbol{B}$, site 2 on Stewart Creek tributary, $\boldsymbol{C}$, site 47 on Merritt Creek tributary, and $\boldsymbol{D}$, site 5 on Keene Creek tributary, Duluth, Minn., area. 
had urban development in the upper half of its watershed, and the Keene Creek tributary had a mix of forest and shrubland but local erosion problems from road drainage.

\section{Potential Response and Sensitivity to Disturbance}

Human activities affect geomorphic processes in Duluth-area streams at multiple spatial scales (tables 5 and 7). Activities include watershed-scale forest clearing for logging, agriculture, or urban development; segment-scale clearing of riparian vegetation for similar purposes, road construction and drainage, and ditching; and reach-scale or local features, such as human-caused channel alterations (straightening, dredging, damming), gravel pits or quarries, road crossings, and hiking trails. Geomorphic conditions in each segment may be affected by upstream changes in the flow regime or sediment inputs, or downstream disturbances such as changes in base level (creation of a reservoir) or straightening (increases slope). Geomorphic processes also may be affected by past activities for which there may be little modern evidence, such as forest clearing, logging, log dams, and log drives following European settlement in the late 1800 s.

Each segment has a known or extrapolated set of geomorphic processes that are presently occurring (table 5). For Lake Superior tributaries in Wisconsin, watershedscale removal of forest vegetation has increased the size of flood peaks and caused increased erosion and sedimentation rates compared to pre-European settlement conditions (Fitzpatrick and others, 1999). Streams in the Duluth area are geologically young and very few are alluvial; thus the textbook concepts of geomorphic stability and potential responses or sensitivity to disturbance that are based on geologically old stream systems in well-developed alluvial valleys may not be applicable (such as development of concave upward profiles described by Hack (1960)).

Example streams that illustrate how geomorphic processes are affected by human activities and disturbance are middle or lower main stems in the Duluth area with entrenched valleys. If their watersheds are completely forested, landslides and bluff erosion would occur episodically where stream meanders intersected valley sides. If a watershed is cleared for urban development, increased runoff and flood peaks would increase stream power, which in turn would increase the erosive potential of the channel, potentially contributing to bank erosion and landslides. Segments with steep slopes and narrow, deep valleys developed in glacial deposits have the most potential for lateral movement and landslides if upstream runoff contributions are increased. Landslide and bluff erosion also can be increased by the creation of gullies from hiking paths, storm drains, and road drainage that concentrate runoff onto erosive or unstable slopes. If a channel is in a bedrock-controlled valley, the likelihood of the occurrence of a landslide is less or minimal during human time scales, but still would be possible in areas that have glacial deposits overlying bedrock. Also, the sensitivity of bedrock channels to disturbance will be less than alluvial channels or channels that flow through glacial deposits.

Another example of geomorphic response to disturbance are middle main stems that flow through glacial deposits but are close to the transition to exposed bedrock, such as at Mission Creek (site 1), Miller Creek (site 34), Chester Creek (site 43), and Lester River (site 23) (figs. 1, 4, and 5). At these sites, Miller and Chester Creeks do not have developed valleys but Mission Creek and Lester River are larger rivers with less resistive bedrock and entrenched valleys. Upstream inputs of water and sediment have changed for all these sites because of land clearing and wetland drainage, most dramatically for Miller Creek because of extensive commercial development in its upper watershed. Downstream, bedrock is a local base-level control, inhibiting incision as a possible geomorphic response to upstream increases in runoff and changes in sediment sources and inputs. The most ubiquitous response in these middle main stems to upstream increases in runoff is channel widening, lateral migration, and avulsion. If the streams have developed valleys, then migration into valley sides causes landslides. Slopes and banks are low enough that overbank sedimentation can result. These streams do not have lateral bedrock controls, and old glacial shorelines and ancient beaches are common in this transition area and deposits from them are easily eroded if they occur along valley sides. Thus, these segments have a high potential for geomorphic change from upstream increases in runoff volumes and flood peaks.

\section{Geomorphic Segment Classification}

Based on the compilation and interpretation of watershed-, segment-, and reach-scale data, the segments were grouped into 15 geomorphic segment categories (GSCs) with similar geomorphic characteristics and processes (fig. 13 and table 9). The categories are primarily based on slope and drainage-network position and secondarily on geologic setting and valley type. Dominant geomorphic processes are summarized for each category. As stated earlier, the classification is based on reach, segment, and 

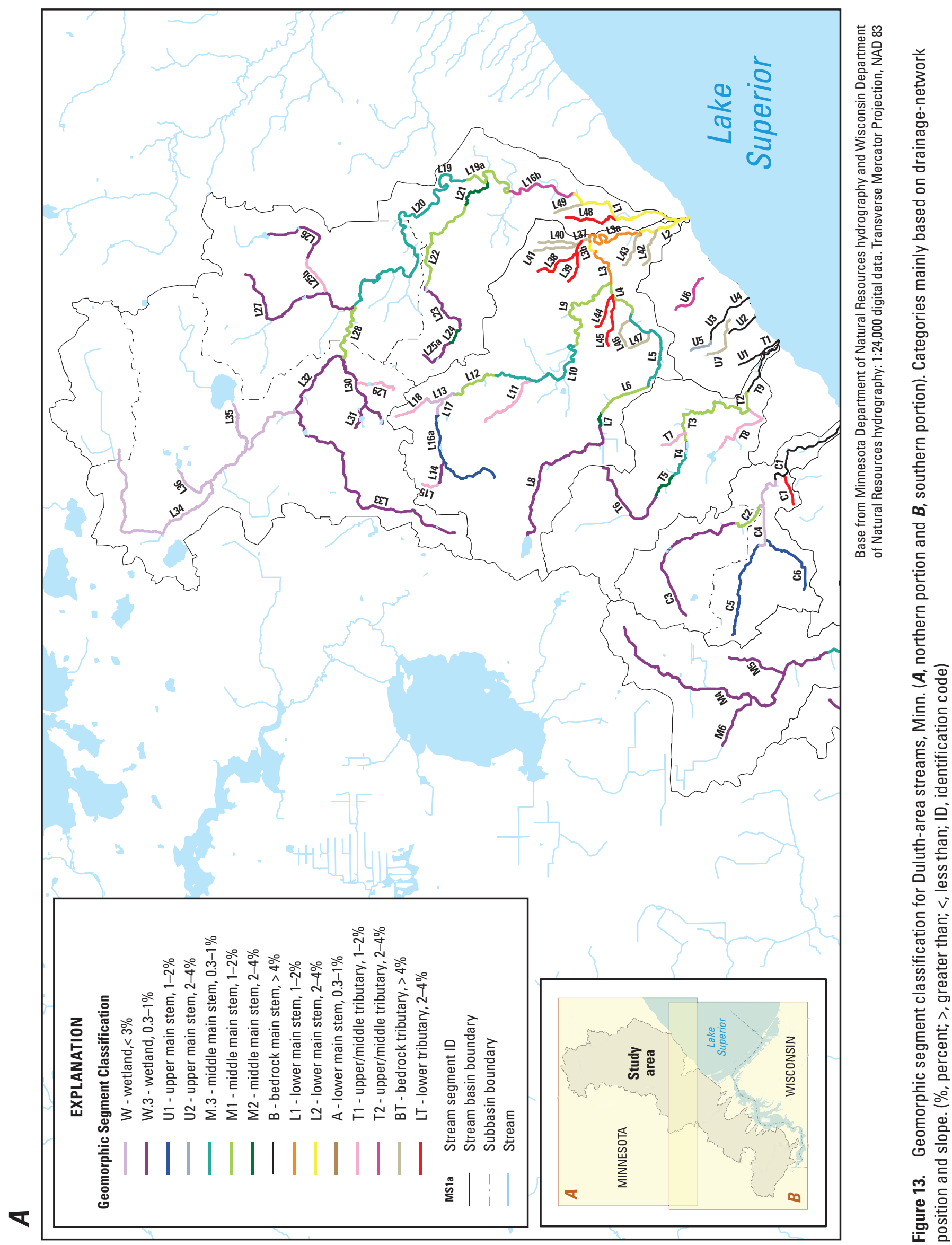


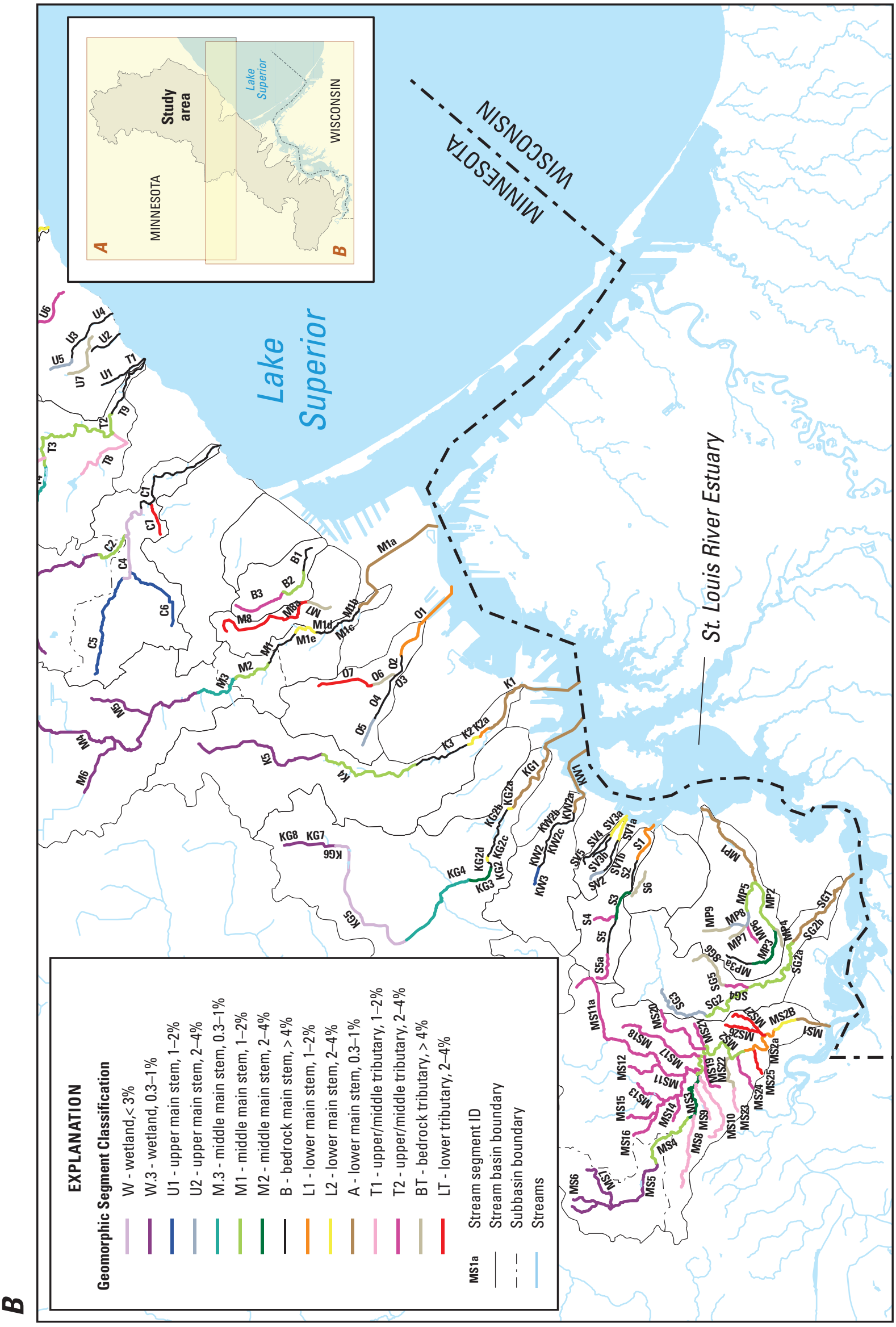




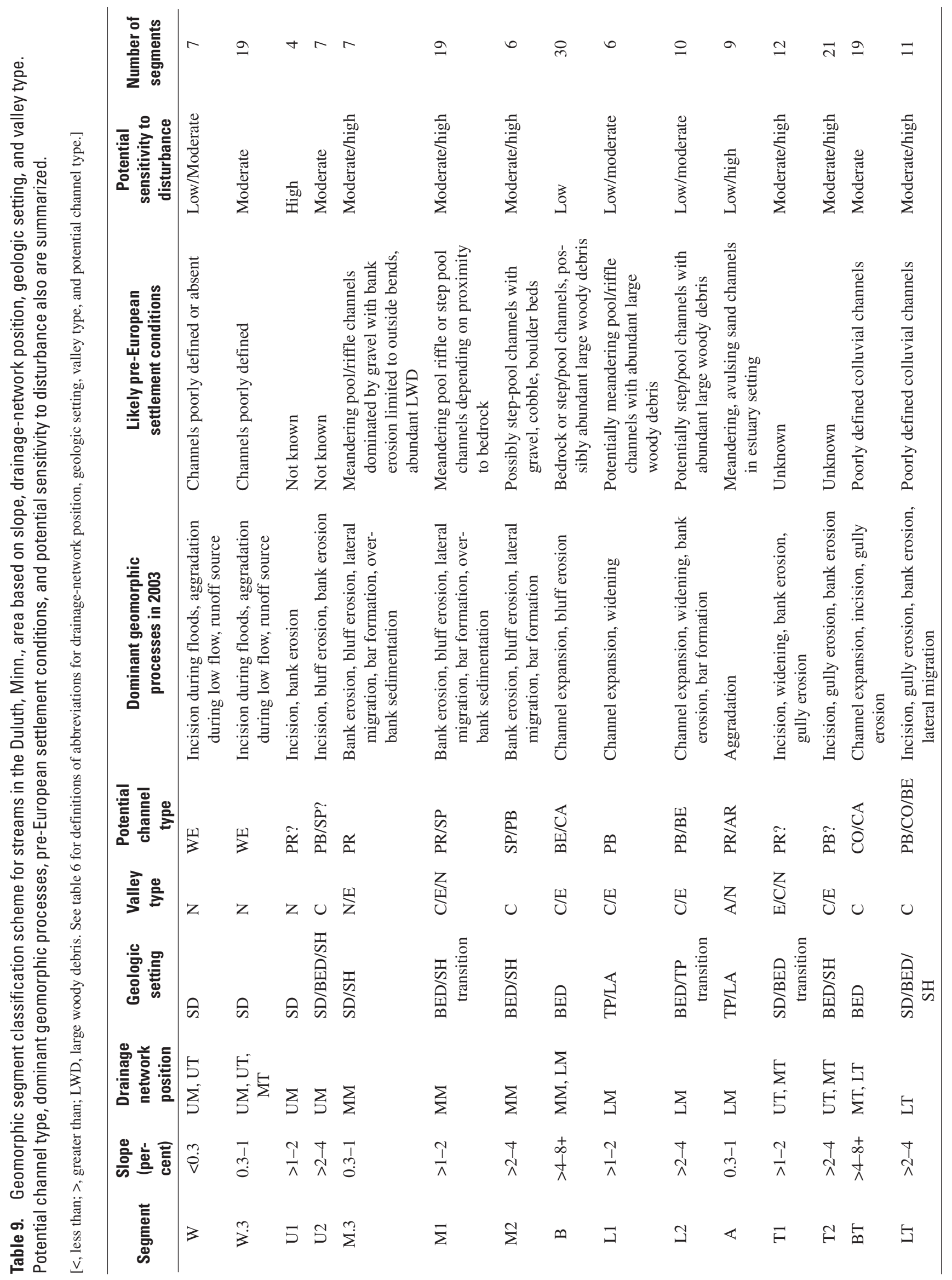


watershed characteristics specific to Duluth-area streams. There may be unknown local disturbance mechanisms that have affected geomorphic processes for some segments that were not field surveyed. This classification is only directly applicable for Duluth-area streams and is useful for showing the importance of linking geomorphic features of a specific reach to drainage-network scale processes. In the Duluth area, streams generally flow perpendicular to their base-level control (Lake Superior or St. Louis River estuary). The boundary between glacial deposits and exposed bedrock, and glacial lake shorelines also run parallel to Lake Superior. Thus, drainage-network position, geologic setting, topography, and valley type are closely related for Duluth-area streams, making the potential sources for and transport of sediment ultimately linked to their position within the drainage network.

\section{Headwater Channels with Gentle Slopes}

Headwater segments with slopes less than 0.3 percent (W) or from 0.3 to 1 percent (W.3) are mainly upper main stems or tributaries to upper main stems in wetlands and lowland areas in the larger watersheds (table 9). The channels are commonly altered by ditching (table 5). Ditching helps speed up runoff to downstream segments with steeper slopes. Many of the channels are bisected by roads and receive runoff from large transportation corridors. Many channels have culvert placement-related problems at road crossings. Channels have water in them and low banks but have little flow between runoff events. Finegrained, organic-rich material accumulates during low flow within the channels; however, the beds of the channels are on parent material, such as glacial lake clay, which gives the appearance that the fine-grained material is flushed out during runoff events.

Headwater streams tend to respond to increases in runoff by widening rather than incision. There is evidence from the GLO notes that the wetland channels are wider now than they were in the mid-1800s, probably from a combination of ditching, road runoff, and historical upland clearing. For example, at site 25 on Lester River, the channel in 1858 was $3 \mathrm{ft}$ wide compared to $18-22 \mathrm{ft}$ wide in 2003. Occasional LWD from banks provides local habitat and sediment trapping but floods are generally not powerful enough to transport large debris.

Presently (2005), these headwater segments generally have low to moderate sensitivity to disturbance because of gentle slopes, but they have some potential to widen with further increases in runoff. Segments with slopes of $0.3-1$ percent are distinguished in the classification from segments with less than 0.3 percent because the potential sensitivity to disturbance may be higher in the channels with greater than 0.3 percent slopes. If runoff is increased through urban development or road drainage, there is a potential that the channels will widen and become greater sources of runoff and fine-grained sediment to downstream segments.

\section{Upper Main Stems with Moderate Slopes}

A small number of upper main stems have greater than 1-2 percent or greater than 2-4 percent slopes (U1 and U2 categories, respectively; table 9). These segments are at the upper end of some of the small watersheds that begin at or near the transition zone between glacial deposits and bedrock, such as Chester Creek (greater than 1-2 percent slopes with no valley development), or in bedrock, such as Merritt Creek (greater than 2-4 percent slopes with confined valleys). Potential causes for geomorphic change include urban development or vegetation clearing, road drainage, and channel alterations. The bedrock segments have higher slopes; however, segments in glacial deposits have a greater potential to respond to increases in runoff through incision or widening. Riparian land cover along the segments varies from urban to forest. If forested, segments may be a source for LWD from the banks.

\section{Middle Main Stems with Gentle or Moderate Slopes}

Middle main stems are divided into three groups based on their slope category (M.3, M1, and M2) (table 9). Middle main stems with greater than 1 to 2 percent slopes are the most common in watersheds within the study area.

Middle main stems with gentle slopes (M.3) are in larger watersheds on supraglacial drift upstream of the bedrock outcrops with no valley development or entrenched valleys. Site 23 on Lester River and site 34 on Miller Creek fall into this category. These segments generally have pool-riffle channel types and are large enough to have some alluvium in channel and overbank areas. The main potential cause for disturbance is upstream increases in runoff. Local disturbances, such as channel alteration and road drainage, also may affect geomorphic conditions. Segments function as sediment source zones through bank erosion, widening and possibly increasing bluff erosion where valleys are developed, zones of sediment transfer, and also zones of sediment accumulation in overbank areas and bar formation. Potential for geomorphic condi- 
tions to change because of upstream increases in runoff is moderate to high. Riparian areas tend to be forested and are a source for LWD, but LWD has a high potential to be transported downstream during floods or form large log jams at bends.

Middle main stems with greater than 1 to 2 percent slopes (M1) are at the transition zone between bedrock and glacial deposits and have confined or entrenched valleys. These segments mainly function as sediment sources and transfer zones, with local storage of sediment in bars downstream of large eroding banks. Similar to M.3 segments, increased runoff from upstream segments is the main potential cause for disturbance, as well as road and storm drainage. There is more potential for landslides and bank erosion than for M.3 segments because of the steeper slopes and more developed valleys. The M1 segments generally have high sensitivity to disturbance unless developed in bedrock. There is no evidence for historical width changes for M1 segments. Forested riparian areas and upstream segments are sources for LWD. At site 43 on Chester Creek, LWD is providing some bank and bar stabilization and sediment trapping, and, occasionally, some pool scour.

Middle main stems with greater than 2 to 4 percent slopes (M2) have confined valleys and are typically developed in transition zones between glacial deposits, bedrock or glacial shorelines. These segments are generally zones of sediment transfer, with some segments also functioning as sediment sources. Potential disturbances are upstream increases in runoff, and local effects of urbanization and road drainage. Possible geomorphic changes from upstream increases in runoff include widening, bank erosion, landslides, lateral migration, and bar formation. Potential for geomorphic change from disturbances ranges from moderate to high, depending on parent material and valley type. Segments with gabbro bedrock have moderate potential, whereas segments with glacial deposits or sedimentary rocks have high potential.

\section{Bedrock Main Stems with Steep Slopes}

Bedrock channels with steep slopes (greater than 4 percent slope) (B) are common and are on steep middle to lower main stems with mainly gabbro bedrock (figs. 4 and 13). The valleys contain little or no alluvium, have little or no flood plain, and channel sides and bottom are composed of bedrock. These segments are mainly source and transport zones for water and sediment. Potential disturbances include increased runoff from upstream segments and feeder tributaries, and local landslides and mass wasting from hiking trails, road drainage, and storm sewers. Most of the sediment from the failures is transported downstream. Increases in runoff and flood peaks result in the expansion of the flow area and scour zone but the channel morphology typically remains the same because of the vertical and horizontal bedrock control. Instead, channel erosion may increase through waterfall recession and knickpoint migration (Tinkler and Wohl, 1998). Pools temporarily fill with fine sediment between floods. A comparison of GLO survey notes and 2003 channel surveys from site 44 on Miller Creek indicates that in 2003 the channel possibly was 3-5 ft wider than in 1858 . Potential for geomorphic change in these segments is low to moderate because the channel morphology is controlled by bedrock.

Presently, the effect of LWD on channel morphology in these reaches is negligible because most of the wood is not large enough to remain stationary during floods. However, LWD may have had more effect on channel morphology prior to European settlement when the size of the LWD was much larger because trees in the immediate riparian zone and upstream segments were larger. It is possible that some cascade or bedrock channels were step/pool prior to European settlement. Bank-derived LWD may alter channel morphology by providing local and temporary scour or lateral migration caused by temporary steps or log jams. A study of LWD in Washington streams suggested that woody debris affects channel morphology, planform, and flood-plain topography (Abbe and Montgomery, 2002); however, woody debris shorter than half the channel width is unstable and provides only temporary storage of sediment (Montgomery and Buffington, 1993).

\section{Lower Main Stems with Moderate Slopes}

Lower main stems with slopes from greater than 1 to 4 percent (L1 and L2) are in Lester River and Mission Creeks and other watersheds in the southwestern part of the study area (fig. 13). These segments generally have entrenched or confined valleys developed in a range of geologic materials (table 5). Many of the channels have human-caused channel alterations and have been straightened or have extensive bank stabilization and grade control. These channels function as zones of sediment transport. Causes for disturbance include increased runoff and sediment from upstream segments, road drainage, concentrated drainage and landslides from storm sewers and hiking trails. Segments that are bedrock controlled have flow expansion from increased runoff. Widening and lateral migration may occur in alluvial channels that have 
not been artificially stabilized. A comparison of channel widths at site 31 on Lester River for 1858 and 2003 indicates that the channel has widened by $5 \mathrm{ft}$ or more. Potential for geomorphic change generally is low to moderate depending on parent material and slope.

\section{Aggradational Main Stems with Gentle Slopes}

Aggradational lower main stems (A) are uncommon in the study area (fig. 13). These segments are on till or lake plain and drain to the St. Louis River estuary (fig. 1). Channels typically are artificially constructed plane-bed channels, such as those at site 38 on Mission Creek. The lower main stem of Sargent Creek potentially is the least affected by human-caused channel alterations. The gentle slopes are conducive to sediment accumulation in overbank areas and in channels. Potential causes for disturbance to these channels include increases in runoff and sediment from upstream areas and concentrated drainage from roads. These streams also are naturally affected by slowly rising water levels in Lake Superior due to regional differences in crustal rebound after glacial ice melted from the region about 10,000 years ago (Larsen, 1994). Potential sensitivity to disturbance ranges from low to high, depending on how channel alteration and hardening has occurred. The source and function of LWD in these segments is unknown. At site 38 on Mission Creek (fig. 1), LWD was rare, with some trees falling in from the banks.

\section{Tributaries with Moderate to Steep Slopes}

As mentioned previously, less time, effort, and field checking was spent on tributaries, especially those that are ephemeral. The majority of the approximately 70 tributary segments are ephemeral and 4 of the 15 GSCs are based on tributaries (T1, T2, BT, and LT; table 9). The divisions for the four categories mainly reflect the potential for the tributaries to incise or form gullies. All four categories are considered source areas for sediment and runoff to main stems. Major causes for disturbances are land clearing, urbanization, concentrated drainage from roads or storm sewers, and gravel pits and quarries. Forested riparian zones provide LWD sources; LWD function likely varies depending on slope.

Tributaries with greater than 1-2 percent slopes (T1) generally flow on glacial deposits and tributaries with greater than 2-4 percent slopes (T2) flow on bedrock or glacial lake shoreline deposits. Valley types for T1 segments include undeveloped, confined, and entrenched, whereas valleys for $\mathrm{T} 2$ segments are confined or entrenched. Many of the T1 and T2 segments are in the Mission Creek watershed, with T2 segments on the eastern side of the watershed because there is more relief coming off of the Duluth gabbro complex and T1 segments on the western side because there is less relief related to the lake plain/till plain and sedimentary bedrock. Both $\mathrm{T} 1$ and T2 segments have moderate to high potential for incision, bank erosion, and landslides. Steeper segments have more potential for erosion unless they are bedrock controlled. Steep segments that intersect glacial lake shoreline deposits have the highest potential for erosion.

Tributaries with confined bedrock valleys (BT) have steep slopes and are also sources of runoff and some sediment to bedrock main stems. Many tributaries are in urban areas. Even though slopes are steep, potential sensitivity to disturbance is moderate because incision and widening are limited by the presence of bedrock in channel margins.

Tributaries to lower main stems (mainly Amity and Mission Creeks) with 2-4 percent slopes are colluvial channels in confined valleys (LT) and are sources for runoff and sediment. These segments have moderate to high potential to incise, widen, and produce gullies and landslides because of their proximity to main stems with entrenched valleys.

\section{Summary and Conclusions}

Streams in 20 watersheds in the Duluth, Minn. area were studied by the USGS in cooperation with the city of Duluth in 2003-04 to identify channel geomorphic characteristics and processes, identify potential causes for geomorphic changes related to disturbance, and develop a classification reflective of characteristics, processes, and potential for geomorphic change. These goals were accomplished by collecting watershed-, segment-, and reachscale geomorphic data from digital maps, historical maps and notes, aerial photographs, and rapid assessments and intensive surveys. Understanding the geomorphic conditions and processes of these streams is important because they relate to overall water and aquatic-resource quality and adjacent plant and animal habitat.

Streams in the Duluth area have been affected by a variety of historical land uses. Main causes of historical and present geomorphic disturbance include logging, agriculture, ongoing urban development, human-caused channel alterations, road and storm sewer drainage, ditching, hiking trails, and gravel pits or quarries. Geomorphic 
responses to these disturbances are dependent on a combination of drainage-network position, slope, and geologic setting. Almost all of the channel segments have undergone some geomorphic changes from past human-related disturbances.

Segments were classified into 15 unique geomorphicsegment categories primarily based on differences in drainage-network position and slope, and, secondarily, based on geologic setting, valley type, and dominant geomorphic processes. The categories accounted for major differences in geology and valley types because stream networks generally are perpendicular to the Lake Superior shoreline, and a major contact between glacial deposits and exposed bedrock parallels the Lake Superior shoreline. The three major bedrock types in the Duluth area have a range in erosion resistance; the least resistant are the Animikie Group sedimentary rocks in the Mission Creek watershed, the most resistant are the Duluth Complex gabbro intrusions in the center of the city; and the Keweenawan volcanic rocks in the Lester and Amity River watersheds are moderately resistive.

Because of insufficient slope or bedrock control, most of the drainage networks in the Duluth area have low to moderate potential for channel change from future disturbance. Some channels cannot incise further because bedrock acts as grade control, but channels can be sediment sources from widening, bank erosion, and landslides (in confined or entrenched valleys). Most of the headwaters segments with gentle slopes at altitudes about 1,200 ft above NAVD27 have been ditched or channelized. Combined with urban development and concentrated road drainage, these segments are primary sources for runoff and secondary sources of sediment to downstream segments. Middle main-stem segments are at altitudes from about 1,200 to $1,000 \mathrm{ft}$ and have moderate slopes in confined or entrenched valleys that are in the contact zone between glacial deposits, bedrock, and glacial-lake shorelines. These segments have a high potential for channel change and are primary sediment sources because they have confined or entrenched valleys with sandy glaciallakeshore deposits.

Below about 1,000 ft, main-stem segments and tributaries generally are affected by bedrock type and structure and have steep slopes and confined or entrenched valleys. Increases in flood peaks usually do not cause incision or widening in bedrock-controlled valleys; instead, the flow and scour area is expanded into riparian zones along the channel boundaries. Feeder tributaries to these main stems have steep, confined valleys and may be sources for runoff and sediment from urban areas or road and storm sewer drainage. Tributaries with glacial deposits overlying colluvium have a high potential for incision, widening, and landslides and mass wasting from valley sides. Aggradational or gentle-sloped lower main stems are in flat areas of till plains or fill between the bedrock bluffs and Lake Superior or the St. Louis River. These channels are mainly artificially constructed or stabilized.

The segment-scale classification developed for this study can be used as a screening tool to distinguish channels that may require extra attention and care in preventing landslides and bank erosion. The classification is an initial step toward a better understanding of the spatial distribution of channels with similar geomorphic processes. The classification can be refined as more reach-scale geomorphic data are collected, especially for tributary segments. The classification could be improved and verified through quantitative geomorphic measurements at additional intensive survey sites and further monitoring at established intensive survey sites.

\section{Acknowledgments}

Todd Bohle (Seattle Public Utilities-Washington) assisted with initial stream characterization and review of historical aerial photos. Kyle Deming (city of Duluth) helped to guide study goals and approach and provided digital maps of Duluth urban features. Meghan Hannon (USGS Minnesota Water Science Center) assisted with field surveys and historical aerial photography interpretation. Christopher Sanocki, Andrea Bergman, and Paul Oduro (USGS Minnesota Water Science Center) provided digital maps of drainage boundaries, bedrock, glacial deposits, land cover, and population density. Jacqueline Hamilton (USGS Minnesota Water Science Center) obtained Government Land Office Survey notes. Meredith Cornett, Brian Schreurs, Thomas Duffus, and Daryl Peterson, all of The Nature Conservancy (Minnesota), are thanked for assistance with digital maps, input on study design, and technical insights. Todd Bohle, Daryl Peterson, David Montgomery (University of Washington), and Timothy Diehl (USGS Tennessee Water Science Center) provided technical reviews. Editorial review was completed by Angel Martin (USGS Illinois Water Science Center). Michelle Greenwood and Jennifer Bruce (USGS Wisconsin Water Science Center) created publication-quality graphics and did the layout design. 


\section{References Cited}

Abbe, T.B., and Montgomery, D.R., 2002, Patterns and processes of wood debris accumulation in the Queets River Basin, Washington: Geomorphology, v. 51, p. 81-107.

Bohle, Todd, 2002, Stream channel assessment of the Caribou, Manitou, and East Branch of the Baptism watersheds: Unpublished report to The Nature Conservancy, January 4, 2002, 34 p.

Camp Dresser and McKee, Inc., 2000, Technical memorandum, assessment of watersheds, City of Duluth: St. Paul, Minn., Camp Dresser and McKee Inc., 35 p.

Eichenlaub, V. L., 1979, Weather and climate of the Great Lakes region: Notre Dame, University of Notre Dame Press, 335 p.

Fitzpatrick, F.A., Waite, I.R., D’Arconte, P.J., Meador, M.R., Maupin, M.A., and Gurtz, M.E., 1998, Revised methods for characterizing stream habitat in the National Water-Quality Assessment Program: U.S. Geological Survey Water-Resources Investigations Report 98-4052, $67 \mathrm{p}$.

Fitzpatrick, F. A., Knox, J. C., and Whitman, H. E., 1999, Effects of historical land-cover changes on flooding and sedimentation, North Fish Creek, Wisconsin: U.S. Geological Survey Water-Resources Investigations Report 99-4083, 12 p.

Gibson, R.J., Haedrich, R.L., and Wernerheim, C.M., 2005, Loss of fish habitat as a consequence of inappropriately constructed stream crossings: Fisheries, v. 30, no. 1, p. 10-17.

Goebel, J.E., Mickelson, D.M., Farrand, W.R., Clayton, L., Knox, J.C., Cahow, A., Hobbs, H.C., and Walton, M.S., Jr., 1983, Quaternary geologic map of the Minneapolis $4^{\circ}$ by $6^{\circ}$ quadrangle, United States: U.S. Geological Survey Miscellaneous Investigations Series, Map 1-1420 (NL-15), scale 1:1,000,000.

Hack, J.T., 1960, Interpretation of erosional topography in humid temperate regions: American Journal of Science, v. 5, p. 258-A, p. 80-97.

Hack, J.T., 1965, Postglacial drainage evolution and stream geometry in the Ontonagon area, Michigan: U.S. Geological Survey Professional Paper 504-B, 40 p.

Harrelson, C.C., Rawlins, C.L., and Potyondy, J.P., 1994, Stream channel reference sites: an illustrated guide to field technique: U.S. Department of Agriculture, Forest Service, General Technical Report RM-245, 31 p.
Hobbs, Howard, 2004, Late Wisconsinan Superior-Lobe deposits in the Superior Basin northeast of Duluth: in Severson, M.J., and Heinz, Julie, Proceedings of the Institute on Lake Superior Geology, 50th Annual Meeting, Field Trip Guidebook, v. 50, p. 86-98.

Knighton, D., 1998, Fluvial forms and processes: New York, Oxford University Press, Inc., 383 p.

Larsen, C.E., 1994, Beach ridges as monitors of isostatic uplift in the upper Great Lakes: Journal of Great Lakes Research, v. 20, n. 1, p. 108-134.

Leverett, Frank, 1929, Moraines and shore lines of the Lake Superior Region: U.S. Geological Survey Professional Paper 154-A, 72 p.

Milfred, C.J., Olson, G.W., and Hole, F.D., 1967, Soil resources and forest ecology of Menominee County, Wisconsin: Madison, Wis., University of Wisconsin, Geological and Natural History Survey, Soil Survey Division, Bulletin 85, Soil Series No. 60: 203 p., 3 pls.

Miller, J. D., Green, J. C., Severson, M. J., Chandler, V. W., and Peterson, D. M., 2002, Geologic map of the Duluth Complex and related rocks, northeastern Minnesota: Minnesota Geological Survey Miscellaneous Map Series M-119, scale 1:200,000.

Minnesota Department of Natural Resources, Division of Forestry, 2002, GAP Land Cover, Tiled Raster: 30-meter resolution.

Minnesota Department of Transportation, 2001, Minnesota Department of Transportation Base Map 2001, Runway: 1:24,000 digital data.

Montgomery, D.R., and Buffington, J.M., 1993, Channel classification, prediction of channel response, and assessment of channel condition: Washington Department of Natural Resources Report TFW-SH10-93-002, Olympia, Wash., variously paginated.

Montgomery, D.R., and Buffington, J.M., 1997, Channelreach morphology in mountain drainage basins: Bulletin of the Geological Society of America, v. 109, p. 596-611.

Montgomery, D.R., and Buffington, J.M., 1998, Channel processes, classification, and response, in Naiman, R.J., and Bilby, R.E., River ecology and management: New York, Springer-Verlag, Inc., p. 13-42.

Munsell Color, 1975, Munsell soil color charts: Baltimore, Munsell Color Division of Kollmorgen Corp., variously paginated.

Schumm, S.A., 1977, The fluvial system: New York, John Wiley and Sons, Inc., $338 \mathrm{p}$. 
Schwartz, G.M., 1949, The geology of the Duluth metropolitan area: Minneapolis, Minn., University of Minnesota, Minnesota Geological Survey Bulletin 33, 136 p.

Soil Survey Staff, 1951, Soil survey manual: U.S. Department of Agriculture, Agricultural Handbook 18, 503 p.

Thorne, C.R., 1998, Stream reconnaissance handbook: Chichester, West Sussex, England, John Wiley and Sons, $133 \mathrm{p}$.

Tinkler, K.J., and Wohl, E.E., 1998, A primer on bedrock channels, in Tinkler, K.J., and Wohl, E.E., eds., Rivers over rock: fluvial processes in bedrock channels: Washington D.C., American Geophysical Union, p. 1-18.

University on Minnesota-Duluth Geology Department, Minnesota Geological Survey, and Minnesota Department of Natural Resources, 1997, Geomorphology of Minnesota, St. Paul, Minn., Minnesota Geological Survey: scale 1:100,000 digital data.

U.S. Census Bureau, 2000, Census data for 2000: U.S. Census Bureau data, accessed February 10, 2006 at http://factfinder.census.gov

Van Riper, D., unpublished data, St. Louis County Census Tracts for 1940, 1960 and 1980: University of Minnesota Department of Geography, digital data.

Wolman, M.G., 1954, A method of sampling coarse riverbed material: Transactions of the American Geophysical Union, v. 35, no. 6, p. 951-956.

Young, H.L., and Skinner, E.L., 1974, Water resources of Wisconsin-Lake Superior Basin: U.S. Geological Survey Hydrologic Investigations Atlas HA-524, 3 sheets. 
8 Printed on recycled paper 